\title{
m6A modification-tuned sphingolipid metabolism regulates postnatal liver development
}

Detian Yuan ( $\nabla$ yuandt@sdu.edu.cn )

Shandong University

Shiguan Wang

Shandong University

Shanze Chen

Shenzhen People's Hospital

Jianfeng Sun

Shandong University

Pan Han

Shandong University

Bowen Xu

Shandong University

Youquan Zhong

Wuhan University

Zaichao Xu

Wuhan University

Peng Zhang

Shandong University

Ping Mi

Shandong University

Cuijuan Zhang

Shandong University

Yuchen Xia

Wuhan University

Shiyang Li

Shandong University

Mathias Heikenwälder

German Cancer Research Center (DKFZ) https://orcid.org/0000-0002-3135-2274

Article 
Keywords: liver, postnatal development, N6-methyl-adenosine, methyltransferase, sphingomyelinase, sphingolipid metabolism, ceramide

Posted Date: February 25th, 2022

DOI: https://doi.org/10.21203/rs.3.rs-1372810/v1

License: (c) (1) This work is licensed under a Creative Commons Attribution 4.0 International License. Read Full License 


\section{$\mathrm{m}^{6} \mathrm{~A}$ modification-tuned sphingolipid metabolism regulates postnatal liver development}

Shiguan Wang, ${ }^{1,8}$ Shanze Chen, ${ }^{2,8}$ Jianfeng Sun, ${ }^{1,8}$ Pan Han, ${ }^{1,8}$ Bowen $\mathrm{Xu},{ }^{1}$ Youquan Zhong, ${ }^{3}$ Zaichao Xu, ${ }^{3}$ Peng Zhang, ${ }^{1}$ Ping Mi, ${ }^{1}$ Cuijuan Zhang, ${ }^{4}$ Yuchen Xia, ${ }^{3}$ Shiyang Li $, 5,6,{ }^{*}$ Mathias Heikenwälder, ${ }^{7, *}$ Detian Yuan ${ }^{1,9,{ }^{*}}$

${ }^{1}$ Department of Biochemistry and Molecular Biology, School of Basic Medical Sciences, Cheeloo College of Medicine, Shandong University, Jinan 250012, China ${ }^{2}$ Shenzhen Institute of Respiratory Diseases, Shenzhen People's Hospital (The Second Clinical Medical College, Jinan University; The first Affiliated Hospital, Southern University of Science and Technology), Shenzhen 518020, China

${ }^{3}$ State Key Laboratory of Virology and Hubei Province Key Laboratory of Allergy and Immunology, Institute of Medical Virology, School of Basic Medical Sciences, Wuhan University, Wuhan 430071, China

${ }^{4}$ Institute of Pathology and Pathophysiology, School of Basic Medical Sciences, Cheeloo College of Medicine, Shandong University, Jinan 250012, China

${ }^{5}$ Department of Gastroenterology, Qilu Hospital, Cheeloo College of Medicine, Shandong University, Jinan 250012, China

${ }^{6}$ Advanced Medical Research Institute, Shandong University, Jinan 250012, China

${ }^{7}$ Division of Chronic Inflammation and Cancer, German Cancer Research Center (DKFZ), Heidelberg 69120, Germany

${ }^{8}$ These authors contributed equally

${ }^{9}$ Lead contact

*Correspondence:

lishiyang@sdu.edu.cn (S.L.)

m.heikenwaelder@dkfz-heidelberg.de (M.H.)

yuandt@sdu.edu.cn (D.Y.) 
Different

organs

undergo distinct

transcriptional, epigenetic

and physiological alterations that guarantee their functional maturation after birth. However, the roles of epitranscriptomic machineries in these processes remain elusive. Here, we show that RNA methyltransferase enzymes Mettl3 and Mettl14 highly express in murine hepatocytes at embryonic and neonatal stages, and gradually decline during the postnatal development. Liver-specific Mettl3 deficiency causes hepatocyte hypertrophy, liver injury and growth retardation. Transcriptomic and N6-methyl-adenosine $\left(\mathrm{m}^{6} \mathrm{~A}\right)$ profiling identify the neutral sphingomyelinase, Smpd3, as a target of Mettl3. Decreased decay of Smpd3 transcripts due to Mettl3 deficiency results in sphingolipid metabolism rewiring, characterized by toxic ceramide accumulation and excessive sphingomyelin hydrolysis, leading to mitochondrial damage and elevated ER stress. Pharmacological inhibition of Smpd3 ameliorates the abnormality of Mettl3-deficent liver. Our findings demonstrate that Mettl $3 / \mathrm{m}^{6} \mathrm{~A}$ fine-tunes sphingolipid metabolism, highlighting the pivotal role of an epitranscriptomic machinery in coordinating metabolic homeostasis and functional maturation during postnatal liver development.

Keywords: liver, postnatal development, N6-methyl-adenosine, methyltransferase, sphingomyelinase, sphingolipid metabolism, ceramide 
The organs of a newborn undergo dramatic tissue growth and maturation to adapt to the functional demands and environmental cues 1-3. These adaptive processes are coordinated by transcriptional and epigenetic mechanisms 1,4,5. Liver is mainly hematopoietic in the embryo, but converts into a major metabolic organ in the adult, concomitant with extensive epigenetic modification ${ }^{6,7}$. Hepatocytes, which are highly proliferative in the fetus, become quiescent, undergo hypertrophic growth, and mature via large-scale changes in gene expression to maintain metabolic homeostasis in the dramatic transitions during and after birth 8,9. Diverse transcriptional and epigenetic mechanisms ensure that lineage specification, tissue growth and functional maturation occur precisely 10-12. Although a wealth of knowledge has been obtained regarding the initial stages of celltype specification and liver organogenesis in embryos ${ }^{10,13,14}$, much less is known about the regulation of liver development and functional maturation during postnatal stages.

RNA modifications have recently been recognized as a new layer of epigenetic regulation, among of which the $\mathrm{N}^{6}$-methyl-adenosine $\left(\mathrm{m}^{6} \mathrm{~A}\right)$ is the most prevalent mRNA modification in eukaryotes ${ }^{15} . \mathrm{m}^{6} \mathrm{~A}$ deposition is catalyzed by the RNA methyltransferase complex containing methyltransferase-like 3 (METTL3), METTL14 and Wilms' tumor 1-associating protein (WTAP). $\mathrm{m}^{6} \mathrm{~A}$ is a reversible modification that can be erased by demethylases, fatmass and obesity-associated protein (FTO) and a-ketoglutarate-dependent dioxygenase alkB homolog 5 (ALKBH5). $\mathrm{m}^{6} \mathrm{~A}$ modification mediates a variety of RNA processing steps, and thus regulates mRNA splicing, mRNA stability, translation efficiency, microRNA processing and XIST-mediated transcriptional repression, impacting a broad range of biological functions ${ }^{16-18}$. Recent studies revealed an evolutionarily conserved role of METTL3 $/ \mathrm{m}^{6}$ A regulatory axis in specifically controlling the degradation rates of immediateearly response genes in response to environmental stimuli ${ }^{19,20}$. Further understanding of how $\mathrm{m}^{6} \mathrm{~A}$ tunes gene expression in different biological processes will refine and advance our understanding of the functions of RNA $\mathrm{m}^{6} \mathrm{~A}$ modification.

The multifaceted functions of $\mathrm{m}^{6} \mathrm{~A}$ are complicated by divergent tissue-specific and contextdependent expression ${ }^{21}$. Recent studies have shown that conditional knockout of Mettl3 leads to cerebellar hypoplasia and axia-like movement disorders ${ }^{22}$. Mettl3 also plays an essential role in the postnatal development of the interscapular brown adipose tissue and adaptive thermogenesis by modulation of Prdm16, Pparg, and Ucp1 expression ${ }^{19}$. In addition, Mett/3-deficient naïve $T$ cells fails to undergo homeostatic expansion and differentiation due to defective degradation of Socs gene family mRNA, whereas Mettl3 deficiency impairs $\mathrm{T}_{\mathrm{FH}}$ differentiation and germinal center responses through accelerated decay of Tcf7 transcripts ${ }^{20,23}$. The writers and erasers likely target different groups of transcripts in different cell types and in different biological processes. Despite the extensive studies of Mettl3 in maintaining the homeostasis of certain organs ${ }^{24,25}$, the roles of Mett| $3 / \mathrm{m}^{6} \mathrm{~A}$-mediated epitranscriptomic control in postnatal liver development remain not well understood.

In this study, we uncovered a new function of the Mett| $3 / \mathrm{m}^{6} \mathrm{~A}$ pathway in postnatal liver development. We observed abundant Mettl3 expression in embryonic and neonatal livers, 
90 and it declined during the postnatal stage in mice. Conditional targeting the Mett/3 gene in 91 mouse liver led to hepatocyte hypertrophy, liver injury and growth retardation. 92 Mechanistically, Mettl3 deficiency impaired the decay of the neutral sphingomyelinase 93 Smpd3, resulting in aberrant accumulation of ceramides, and consequent mitochondrial 94 damage and elevated ER stress. Our findings highlighted the importance of 95 epitranscriptional machinery in metabolic homeostasis for postnatal liver development. 


\section{Mett|3 is enriched in embryonic and neonatal livers and declines during postnatal liver development}

To interrogate how the liver develops and matures into the major metabolic organ after birth, we analyzed a publicly available transcriptomic dataset of livers from C57BL/6J mice across twelve time points that covered the whole period of liver development from late embryonic stage (E17.5) to adult (postnatal Day 60) ${ }^{8}$. Principal component analysis (PCA) showed that liver samples from different ages were separated in a time-dependent manner (Fig. 1a), suggesting that neighboring time points have similar expression snapshots, and liver transcriptome was reprogramed gradually during the postnatal developmental process. $k$-means clustering analysis further categorized the transcriptome into eight clusters. Time-course analysis was conducted to investigate their expression dynamics (Fig. 1b and 1c, Extended Data Fig. 1b and Extended Data Fig. 1c and Supplementary Table 1-3). Most known fetal liver-specific genes, including Afp and Igf2, accumulated in sub-cluster 4 and 5, were highly expressed in embryonic and neonatal livers and downregulated with age (Fig. 1d, Extended Data Fig. 1b and Extended Data Fig. 1c, and Supplementary Table 1). This group overrepresented genes associated with cell division, cell cycle, DNA replication and cytoskeleton organization (e.g. Ccne1, Cdk4, E2f1, Mki67 and Top2a), supporting that cell proliferation during embryonic liver organogenesis was transcriptionally silenced (Fig. 1b-1d). In addition, this downregulating group also contained genes involved in DNA confirmation change, histone modification and chromatin binding, indicating a close correlation between epigenetic reprogramming and fetal-to-adult liver maturation (Fig. 1b-1d). The expression pattern of sub-cluster 7 and 8 was constantly upregulating after birth, with genes involved in metabolism of lipid, carbohydrate and small molecules (Fig. 1b and 1d, Extended Data Fig. 1b and Extended Data Fig. 1c, and Supplementary Table 2). Typical genes of this group included Apoa4, Cyp2e1, Cyp2f2, and Aldh1a1, reflecting the gradual establishment of the metabolic functions during postnatal liver development (Fig. 1d). Sub-cluster 1 and 2 overrepresented genes associated with tissue morphogenesis, blood vessel development and ECM organization, e.g. Wnt5a, Wnt9b, Thy1, Tek, Flt1 and Bmp10, specifically upregulated after birth and declined after week 2 , in line with their roles in the early stage of postnatal liver development (Fig. $1 \mathrm{~b}$ and 1d, Extended Data Fig. 1b and Extended Data Fig. 1c, and and Supplementary Table 3). Notably, selective enrichment of genes and pathways involved in RNA processing were observed in the downregulating gene set. Analysis of the differentially expressed genes further highlighted that both of the core enzymes of the $\mathrm{m}^{6} \mathrm{~A}$ methyltransferase complex, Mett/3 and Mett/14, declined along with the developmental process (Fig. 1d).

To verify these results, we measured the expression levels of Mettl3 and Mettl14, as well as other factors involved in $\mathrm{m}^{6} \mathrm{~A}$ biology in mouse livers at different ages. qRT-PCR analyses revealed that Mett/3, Mettl14 and Fto gradually downregulated after birth, accompanying the upregulation of genes implicated in hepatic metabolism, while Afp was shut down at week 2 after birth (Fig. 1e and Extended Data Fig. 2a). mRNA levels of Wtap were higher in neonatal and adolescent livers, whereas the expression of the demethylase 
postnatal liver development (Fig. 1e and Extended Data Fig. 2a). As the fetal liver is temporarily home to a sizable population of hematopoietic cells, to determine if the expression changes of Mettl3 might be attributable to their emigration from the liver, we performed immunohistochemistry ( $\mathrm{IHC})$ analysis and confirmed hepatocytes as the major cell type expressing Mettl3 in the embryonic and neonatal livers, but not hematopoietic cells (Fig. If and Extended Data Fig. 2b). In line with the changes at mRNA level, expression of Mettl3 in hepatocytes was much lower in the adult liver than that in the embryonic and neonatal livers, compared to retention of staining in the internal positive non-parenchymal cells (Fig. $1 \mathrm{f}$ and Extended Data Fig. 2b). Moreover, as in mice, METTL3 expression in human fetal livers was also higher than that in adult livers (Extended Data Fig. 2c). Given that the postnatal period is a critical time-window when hepatocytes establish their functional maturation, and $\mathrm{m}^{6} \mathrm{~A}$ is essential for cell fate patterning ${ }^{26,27}$, we hypothesized that Mettl3 might have an important role in postnatal liver development.

\section{Hepatic Mettl3 deficiency induces hepatocyte hypertrophy, liver injury and growth retardation}

To investigate the role of $\mathrm{m}^{6} \mathrm{~A}$ modification in postnatal liver development, we generated liver-specific Mettl3 knockout mouse model (hereafter referred to as Mettl $3^{\Delta \text { hep }}$ ) by crossing Mett/3-floxed mice (Extended Data Fig. 3a) with mice expressing Cre recombinase under the control of the albumin promoter and enhancer ${ }^{28}$. qRT-PCR and immunoblotting analysis demonstrated efficient Mettl3 depletion (Fig. 2a and 2b). Moreover, by RNA dot blot analysis, we observed dramatically reduced RNA $\mathrm{m}^{6} \mathrm{~A}$ levels in Mettl $3^{\text {Ahep }}$ compared with control livers (Fig. 2c). IHC confirmed hepatocyte-specific Mettl3 depletion in Mett|3 ${ }^{\Delta}$ hep livers, while its expression in non-parenchymal cells was unaffected (Fig. 2d). Although the Mett| $3^{\triangle \text { hep }}$ mice were born at the expected Mendelian ratios, we noted growth retardation during postnatal development (Fig. 2e and 2f). As signs of liver injury, Mett|3 ${ }^{\Delta}$ hep mice displayed moderately increased serum alanine aminotransferase (ALT) and AST (Fig. $2 \mathrm{~g}$ ) and pale livers with rough surfaces (Fig. 2h).

On light microscopic examination, hepatocyte hypertrophy, with the cell and nuclear sizes both enlarged, was evident in Mettl $3^{\text {Ahep }}$ livers (Fig. 2i and 2j). Hepatocellular injury, characterized by ballooning degeneration, was seen as early as 4 weeks after birth (Extended Data Fig. 3c). Both TUNEL and cleaved Caspase-3 (cl-Casp3) IHC revealed a significantly increased cell apoptosis in Mettl $3^{\Delta \text { hep }}$ livers (Fig. 2k). The expression of several cell apoptosis mediators cl-Casp3, BAX, cytochrome C (Cyt-c) and Hmgb1 was dramatically increased (Fig. $2 \mathrm{I}$ and $2 \mathrm{~m}$ ). Analysis of the pro-survival signaling revealed that

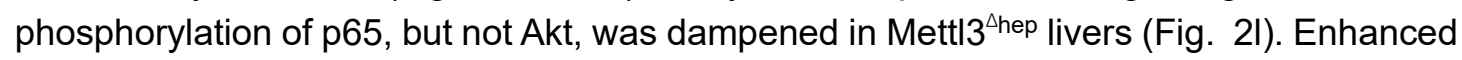
cell death led to immune cell infiltration in the Mettl $3^{\Delta \text { hep }}$ mice, as evidenced by CD45, F4/80, CD3 and B220 staining (Extended Data Fig. 3d), and upregulation of pro-inflammatory cytokines and chemokines (Extended Data Fig. 3e). As compensatory responses to liver injury, hepatocyte proliferation and a ductular reaction were evidenced by increased expression of Ki67, CK19 and EpCAM, accompanied by upregulation of cell-cycle-related genes Ccne1, Ccna2 and Pcna (Fig. 2I, 2n, and Extended Data Fig. 3e).

In parallel, we delivered adeno-associated virus serotype 8 (AAV8) carrying a thyroid- 
binding globulin (TBG) promoter driven Cre recombinase into adult Mett/3-floxed mice (hereafter referred to as AAV8-Cre Mett//f/ffl $)$ (Extended Data Fig. 3f). Immunoblotting and $\mathrm{IHC}$ analysis confirmed efficient Mettl3 knockout (Extended Data Fig. $3 f$ and Extended Data Fig. 3g). However, unlike Mettl $3^{\Delta \text { hep }}$ mice, AAV8-Cre Mett/ $3^{\text {fl/fl }}$ mice did not shown any abnormality during the time of experiments (Extended Data Fig. 3h-j). Histological analysis did not show any sign of hepatocellular injury either (Extended Data Fig. 3k). Together with the above observation that Mettl3 expression declines in the adult liver, these results indicate that Mett|3 has specific roles in postnatal liver development.

To determine whether the defects during the early development in Mettl $3^{\Delta \text { hep }}$ mice might have impact on liver functionality, we subjected 10 -week-old Mettl $3^{\text {Lhep }}$ mice to DDCinduced liver fibrosis or partial hepatectomy. Mettl $3^{\Delta \text { hep }}$ deletion exacerbated DDC-induced liver fibrosis as evaluated by higher serum levels of ALT and AP, elevated expression of hepatic procollagen and increased number of $\alpha$-smooth muscle actin ( $\alpha$ SMA)-positive myofibroblasts (Extended Data Fig. 4a-e). Consistently, Mettl $3^{\Delta \text { hep }}$ mice had higher mortality following 2/3 partially hepatectomy, and extensive multilobular hemorrhagic necrosis as evidenced by histology (Extended Data Fig. 4f, 4g). These results confirm that Mett| $3^{\triangle \text { hep }}$ mice had liver injury that could be further exacerbated upon additional stress stimuli.

Hepatic Mett|3 deficiency leads to metabolic reprogramming featured by deregulated sphingolipid biosynthesis

To identify potential target genes that are closely associated with the developmental defect, we compared the transcriptomes of Mettl $3^{\text {Ahep }}$ versus WT livers. Differential expression analysis (FDR $<0.01$, Fold change $>1.5$ ) identified 833 upregulated genes and 654 downregulated genes (Fig. 3a). Gene ontology (GO) analysis revealed that the upregulated genes in Mettl $3^{\triangle \text { hep }}$ livers were functionally enriched in terms associated with chromatin segregation and nuclear division (Fig. 3b), consistent with the enhanced compensatory proliferation observed in vivo. The downregulated genes were enriched in metabolic pathways, in particular fatty acid and xenobiotic metabolism (Fig. 3c and Extended Data Fig. 5a-c), indicating that the functional maturation was not properly established in Mettl $3^{\Delta}$ hep livers. In line with the most recent report that Mettl3 depletion protected mice against HFD-induced metabolic syndrome ${ }^{29,30}$, Mettl $3^{\text {Lhep }}$ mice fed with HFD from the age of 10 weeks for 5 months exhibited reduced body weight gain, lower serum triglyceride level and improved insulin sensitivity (Extended Data Fig. 5d-f). H\&E and oil red staining confirmed reduced steatosis and lipid accumulation in Mettl $3^{\Delta \text { hep }}$ livers compared to WT livers (Extended Data Fig. 5g-h). Contrary to the protective effect of Mettl3 deletion against HFDinduced metabolic disorder, liver injury was observed in Mettl $3^{\triangle \text { hep }}$ mice, which might not be explained by the downregulation of fatty acid metabolic pathways.

To interrogate the mechanisms underlying the observed liver phenotype in Mettl $3^{\Delta \text { hep }}$ mice, we examined the transcriptomic data by Kyoto Encyclopedia of Genes and Genomes (KEGG) pathway enrichment analysis in detail. KEGG analysis again revealed extensive metabolic reprogramming in Mettl $3^{\triangle \text { hep }}$ livers, among of which the sphingolipid metabolism pathway was the most top-ranked (Fig. 3d, 3E, Extended Data Fig. 6). Given that 
perturbation of sphingolipid metabolism can elicit tissue dysfunction characterized by apoptotic cell death ${ }^{31-33}$, we then focused on genes associated with sphingolipid metabolism, and confirmed the upregulation of transcripts implicated in sphingolipid metabolism in Mettl $3^{\text {Ahep }}$ livers by qRT-PCR (Fig. $3 f$ and $3 g$ ). Notably, mRNA levels of Smpd1 and Smpd3, which catalyze the ceramide generation by hydrolysis of sphingomyelin (SM), and Sptlc1/2, encoding the rate-limiting enzymes of de novo ceramide synthesis, were strongly upregulated in Mettl $3^{\triangle \text { hep }}$ livers (Fig. 3g). Upregulation of Smpd1, Smpd3, and Sptlc2 was further confirmed at protein level by immunoblotting (Fig. 3h) and IHC (Fig. 3i-3k). Among of these, Smpd3, the plasma membrane-tethered sphingomyelinase, was the most strongly upregulated.

\section{Mettl3 regulates $S m p d 3$ expression by $\mathrm{m}^{6} \mathrm{~A}$-mediated RNA decay}

To identify the gene(s) under the control of $\mathrm{m}^{6} \mathrm{~A}$ methylation, we performed methylated RNA immunoprecipitation combined with high-throughput sequencing $\left(\mathrm{m}^{6} \mathrm{~A}-\mathrm{seq}\right)$ on Mettl3 $\triangle$ hep and WT livers. The $m^{6} A$ writer complex preferentially deposits $m^{6} A$ on the consensus sequence, $D R A * C H(D=G / A / U, R=G / A, A *=m 6 A, H=U / A / C)$, and the $m^{6} A$ modification motif was reduced upon Mettl3 knockout (Fig 4a). $\mathrm{m}^{6} \mathrm{~A}$ levels on the $3^{\prime}$ ends of mRNAs from coding genes was significantly reduced in Mettl $3^{\triangle \text { hep }}$ livers, resulting in the altered distribution of $\mathrm{m}^{6} \mathrm{~A}$ across the transcript body (Fig. $4 \mathrm{~b}$ and $4 \mathrm{c}$ ). Additionally, we found a significant increase in $\mathrm{m}^{6} \mathrm{~A}$-marked mRNAs in Mett $3^{\text {Ahep }}$ livers compared to WT livers, as reflected by a right shift in the cumulative fraction of these transcripts compared with non$\mathrm{m}^{6} \mathrm{~A}$-marked transcripts $\left(P=2.2 \times 10^{-16}\right.$, Fig. $\left.4 \mathrm{~d}\right)$. This is in line with the previous studies showing that $\mathrm{m}^{6} \mathrm{~A}$ destabilizes $\mathrm{mRNAs}$ and inhibition of $\mathrm{m}^{6} \mathrm{~A}$ deposition on these transcripts leads to increased mRNA stability.

To correlate the gene expression level with the $\mathrm{m}^{6} \mathrm{~A}$ modification level, we plotted the $\mathrm{m}^{6} \mathrm{~A}$ peak data against the RNA-seq data, which divided the differentially expressed genes into four groups (Fig. 4e). Overlay of upregulated genes from RNA-seq with $\mathrm{m}^{6} \mathrm{~A}$ hypomethylated genes from $\mathrm{m}^{6} \mathrm{~A}$-seq identified 714 targets (Fig. 4e). Pathway analysis again revealed enrichment in genes involved in sphingolipid metabolism (Fig. 4f). Among of these, Smpd3 had the lowest $\mathrm{m}^{6} \mathrm{~A}$ modification level and the highest mRNA abundance (Fig. 4g). We conducted $\mathrm{m}^{6} \mathrm{~A}-\mathrm{RNA}$ immunoprecipitation (MeRIP) combined with qRT-PCR and confirmed that the $\mathrm{m}^{6} \mathrm{~A}$ levels on Smpd3 mRNA but not the other transcripts tested were significantly decreased in Mettl $3^{\triangle \text { hep }}$ livers (Fig. 4i).

Mett|3-m $\mathrm{m}^{6} \mathrm{~A}-Y$ thdf2 axis destabilizes $\mathrm{m}^{6} \mathrm{~A}$-marked transcripts ${ }^{34}$. To further test whether the up-regulation of Smpd3 was due to decreased degradation, we performed RNA decay assay by treating either WT or Mettl3-deficient primary hepatocytes with actinomycin D and measured the abundance of Smpd3 transcripts over time (Fig. 4j). As expected, degradation of Smpd3 mRNA was significantly impaired in the absence of Mettl3 (Fig. 4j). Furthermore, siRNA-mediated knockdown of Ythdf2 - the $\mathrm{m}^{6} \mathrm{~A}$ reader that regulates RNA decay 35,36 - delayed Smpd3 mRNA degradation, while the degradation of the other transcripts tested was largely unaffected (Fig. 4k). Collectively, these data demonstrate that Mettl3-mediated m6A modification regulates Smpd3 expression by accelerating its 
mRNA decay.

Mett|3 deficiency in hepatocytes results in ceramide accumulation, mitochondrial damage and ER stress

269

270

271

272

273

274

275

276

277

278

279

280

281

282

283

284

285

286

Sphingomyelinases activated by stress stimuli can elicit tissue dysfunction by acutely liberating ceramides ${ }^{37,38}$. To further understand the association between disrupted sphingolipid homeostasis and Mettl3 deficiency-induced liver injury, we performed lipidomic profiling of Mettl $3^{\Delta \text { hep }}$ versus WT livers. The PCA showed a pronounced separation of the Mettl $3^{\Delta \text { hep }}$ livers from the WT livers based on their lipid profiles (Fig. 5a), in accordance with the prominent alterations in cellular metabolism revealed by RNA-seq analysis (Fig. 3). Quantification of sphingolipid species revealed that SM was significantly reduced in Mettl $3^{\Delta \text { hep }}$ livers (Fig. 5b-5d). The relative amount of several abundant SM species changed significantly, including SM (d40:1), SM (d41:1), SM (d42:1) and SM (d42:2) (Fig. 5c). Concurrent with the downregulation of SM, the levels of ceramides were significantly increased in Mettl $3^{\text {thep }}$ livers (Fig. $5 \mathrm{~b}, 5 \mathrm{e}$, and $5 \mathrm{f}$ ), with the most pronounced changes in ceramide (d18:1/16:0) and ceramide (d18:1/24:1) (Fig. 5e), two of the most abundant naturally occurring ceramides in mammalians. Increased levels of individual species of ceramides were further confirmed by high performance liquid chromatography coupled with tandem mass spectrometry (HPLC-MS/MS), which showed an upregulation of ceramide (d18:1/16:0) by 3-fold and ceramide (d18:1/24:1) by 2-fold (Fig. 5g). Together with the transcriptomic data, these results suggest that Mettl3 deficiency in hepatocytes disrupts sphingolipid homeostasis, resulting in ceramide accumulation.

Ceramides can modulate physical properties of cellular membranes such as mitochondrial and ER. Electron microscopy revealed structural defects of mitochondria in Mettl3 deficient hepatocytes, characterized by low electron dense mitochondrial matrix and fragmented cristae, indicative of mitochondria degeneration (Fig. 6a). In addition, key features of ER stress, swollen endoplasmic reticulum (ER) and dilation of the perinuclear cisternae, were evident (Fig. 6b and Extended Data Fig. 7a). In accordance with the profound structural alterations, mitochondrial membrane potential, as measured by JC-1-staining, was decreased in the primary hepatocytes isolated from Mettl $3^{\text {Ahep }}$ versus WT mice (Fig. 6c, 6d, Extended Data Fig. 7b, and Extended Data Fig. 7c). These structural and functional changes were accompanied by marked elevation of cellular reactive oxygen species (ROS) and mitochondrial unfolded protein response (UPR) markers including Ppargcla and Dele1 (Fig. 6e and 6f). Analysis of the three branches of the ER UPR revealed that the protein kinase R-like ER kinase (PERK) pathway was specifically activated, as shown by upregulation of phosphorylated elF2 $\alpha$ (p-elF2 $\alpha$ ), Atf3 and CHOP (encoded by Ddit3), whereas the expression of Ire1 $\alpha$, Xbp1 or Atf6 was not uniformly changed (Fig. $6 \mathrm{~g}$ and $6 \mathrm{~h}$ ). In addition, downstream targets of Atf3, including Eif2ak, Trib3, and Bcl2, increased in Mettl3 $3^{\text {hep }}$ livers compared with control livers (Fig. 6h). Presence of ER stress in Mettl3 deficient hepatocytes was further confirmed by IHC of BIP and p-eIF2 $\alpha$ (Fig. 6i and 6j). These results collectively indicated mitochondrial damage and ER stress were provoked in Mettl3-deficient hepatocytes, which might account for the phenotype observed in the Mett|3 $3^{\text {hep }}$ mice. 

and liver injury in Mettl $3^{\Delta \text { hep }}$ mice

310 To assess whether deregulated ceramide metabolism was casually linked to the liver injury 311 induced by Mettl3 knockout, we treated Mettl $3^{\text {Ahep }}$ mice with GW4869, an antagonist of 312 Smpd3, starting on postnatal day 42 for 3 weeks (Fig. 7a). GW4869 abolished the upregulation of serum ALT and AST levels, implying the reversal of Mettl3 deficiencyinduced liver damage (Fig. 7b). Morphological and histological analysis further confirmed that GW4869 treatment restored liver architecture (Fig. 7c and Extended Data Fig. 8a). Importantly, GW4869 reversed Mettl3 deficiency-induced accumulation of ceramides (Fig. 7d). Ultrastructural analyses demonstrated that mitochondrial and ER abnormalities in Mettl $3^{\Delta \text { hep }}$ livers were greatly improved upon Smpd3 inhibition, as evidenced by alleviation of mitochondrial degeneration, ER swollen and perinuclear cisternae dilation (Fig. 7e). It was of interest to mention that Mettl3 deficient hepatocytes treated with GW4869 still displayed enlarged cell size (Fig. 7f), suggesting that this phenotype is most likely attribute to other uncovered mechanism(s). In line with the alleviation of liver damage, GW4869 abrogated the induction of apoptosis (Bax, cl-Casp3, Hmgb1 and cytC), mitochondrial UPR (Apaf1, Oma1, Ppargcla and Cox4i1) and ER UPR (Atf3, p-elF2 $\alpha$ and BIP) (Fig. 7g-7i and Extended Data Fig. 8b-8d). Consistently, the induction of inflammation and compensatory proliferation were also attenuated in Mettl $3^{\triangle \mathrm{hep}}$ mice treated with GW4869 (Extended Data Fig. 8e-8h). Additionally, Mettl $3^{\Delta \text { hep }}$ mice did not benefit from the treatment with either the Sptlc2 inhibitor myristicin or the Smpd1 inhibitor imipramine (Extended Data Fig. 8a). Taken together, these data suggest that upregulation of Smpd3 and the resultant accumulation of ceramides dictate the metabolic disorder and liver damage caused by Mettl3 deficiency. 
The in vivo functions of Mett $3 / \mathrm{m}^{6} \mathrm{~A}$ regulatory axis in mammals have just started to be elucidated by using the Mettl3 conditional knockout mouse models. Here, we reported a physiological role of Mettl3-mediated $\mathrm{m}^{6} \mathrm{~A}$ modification in liver metabolic homeostasis during postnatal development. Hepatic metabolism is dynamically regulated after birth by a complex transcriptional network implicated in energy metabolism including Hnf4a, Ppara, LXR, Srebp1/2, Sirt1 and Fxr ${ }^{39}$. By using transcriptomic, $\mathrm{m}^{6} \mathrm{~A}$ profiling and lipidomic analysis, we provided evidence that Mett $3 / \mathrm{m}^{6} \mathrm{~A}$-mediated epitranscriptomic regulation is involved in maintaining metabolic homeostasis by fine-tuning ceramide biosynthesis, highlighting the epitranscriptomic machinery as a gatekeeper of hepatic sphingolipid metabolic homeostasis during postnatal liver development.

The roles of Mettl3 in mammalian liver appear to be controversial according to recent studies. The Mettl3- $\mathrm{m}^{6} \mathrm{~A}$ pathway has been implicated in the crosstalk between hepatic circadian networks and lipid metabolism by regulating the decay of the nuclear receptor peroxisome proliferator-activator $\alpha(\mathrm{PPaR} \alpha)^{40}$. Mett|3 expression and $\mathrm{m}^{6} \mathrm{~A}$ level increased in the livers of mice with high fat diet (HFD)-induced non-alcoholic fatty liver disease (NAFLD) 29,30. Hepatocyte-specific knockout of Mett/3 significantly reduced lipid accumulation and improved insulin sensitivity, thus alleviating HFD-induced metabolic syndromes. Contrary to the pro-steatotic effects of Mettl3 in HFD condition, we and others observed that lipid metabolic abnormality unexpectedly increased in Mettl3 deficient liver 41. The study of Barajas et al., which completed at the same time as this work, reported similar observations, e.g., hepatocyte ballooning, ductular reaction and focal inflammation, when Mett/3 was deleted during embryonic and neonatal development using Alb-Cre ${ }^{41}$, whereas liver injury was not noticed when Mett/3 deletion was induced in adult hepatocytes using AAV8-Cre in Mett//f//fl mice ${ }^{30}$. Our integrated transcriptomic and lipidomic profiling of the Mettl $3^{\text {hep }}$ livers further revealed cellular metabolism rewiring, indicative of defective functional maturation due to Mettl3 deletion driven by Alb-Cre. Together, these independent investigations suggested that knockout of Mett/3 in hepatocytes during developmental stage generates more severe cellular injury than that in adult stage. The phenotypic differences in hepatocytes owing to Mettl3 deletion at different time points might be explained by the fact that the functions of $\mathrm{m}^{6} \mathrm{~A}$ modification are more critical for cell state transitions where a transcriptome switch is typically needed (Ivanova et al., 2017, Li et al., 2018, Zhao et al., 2017). As the functional maturation of hepatocytes during the postnatal period is accomplished by turning genes on and off, hepatocytes in neonatal livers may rely on Mett|3/m6A pathway for fine-tuning the extensive transcriptomic remodeling. These findings reinforce the notion that Mettl3 orchestrates different subsets of genes in different contexts (e.g., developmental stage versus adult stage, and HFD condition versus normal diet condition), and also highlight the complexity of epitranscriptomic regulation in cellular metabolism.

Sphingolipid metabolism, and the generation of ceramide in particular, have been increasingly investigated concerning their roles in cellular processes such as growth arrest, apoptosis, necroptosis, mitophagy, and senescence. The first evidence of ceramides as obligate intermediates that induce tissue failure was obtained by the Unger laboratory in 
1990s, showing that ceramides produced by de novo synthesis drives apoptosis, beta-cell failure and diabetes. The involvement of ceramides was revealed in a wide range of human pathogenesis characterized by apoptotic cell death, including radiation-induced injury, atherosclerosis, Alzheimer's dementia, cystic fibrosis, asthma and heart failure ${ }^{42-49}$. There are diverse molecular mechanisms involved in the modification of ceramide production and degradation. For example, TLR4, activated by saturated fatty acids, upregulates enzymes in the de novo ceramide biosynthesis in an IKKß-dependent manner, linking exogenous fats to the disruption of insulin action ${ }^{50}$. FGF21 was found to drive beneficial metabolic state by selectively lowering ceramide levels through an FGF21-adiponectin-ceramidase axis ${ }^{51}$. Gut microbiota also participate in controlling the rate of ceramide synthesis by a bile acid/intestinal farnesoid $X$ receptor (FXR) signaling axis ${ }^{52}$. Although the regulation of the enzymes responsible for ceramide synthesis or degradation has been intensely studied at the transcriptional level, the post transcriptional mechanisms controlling the balanced expression of these key factors remain obscure. Our findings reveal the engagement of Mettl3 in regulating Smpd3 expression via $\mathrm{m}^{6} \mathrm{~A}$-dependent RNA decay, in line with the notion that Mettl $3 / \mathrm{m}^{6} \mathrm{~A}$ axis is required for the timely elimination of immediate-early response genes driven by environmental stimuli 20 .

Taken together, our work demonstrates a Mettl3-mediated epitranscriptomic control of ceramide metabolism during postnatal liver development. Given that ceramides are amongst the most deleterious lipid metabolites, the accumulation of which dictates cellular dysfunction, we postulate that a fast and flexible regulation of Smpd3 by Mettl $3 / \mathrm{m}^{6} \mathrm{~A}$ protects hepatocytes from excessive ceramide accumulation and metabolic disruption. Our finding that fine-tuning ceramide metabolism by Mett $3 / \mathrm{m}^{6} \mathrm{~A} / \mathrm{Smpd} 3$ axis in hepatocytes expands the roles of $\mathrm{m} 6 \mathrm{~A}$ modification in sphingolipid homeostasis during postnatal liver development. 


\section{Lead contact}

402 Further information and requests for resources and reagents should be directed to the lead contact, Detian Yuan (yuandt@sdu.edu.cn).

Materials availability

This study did not generate new unique reagents.

Data and code availability

RNA-seq and MeRIP-seq data reported in this study have been deposited in the National Center for Biotechnology Information (NCBI) Sequence Read Archive (SRA) database under accession number PRJNA771919.

\section{EXPERIMENTAL MODEL AND SUBJECT DETAILS}

\section{Animals}

Mett// $/^{f / f t}$ mice were generously provided by Shanze Chen. Mett//ff/fl mice (C57BL/6J) were hybridized with mice expressing Cre recombinase (Cre) under the control of the albumin promoter and enhancer (Alb-Cre) to generate liver-specific Mett/3 knockout mice. Mett//f//fl and Alb-Cre mice were all used in a C57BL/6 background. AAV8 mediated Cre expression in hepatocytes were done by i.v. injection of $2.5 \times 10^{11}$ viral genomes of AAV8-TBG-Cre into 10-week-old male mice. Genotyping was performed with the following primers: Mett/3F, 5' -TAGTGCTGTGCCTTTCTTAG -3', Mett/3-R, 5'- TTAAACTGACTGCCTCCATA -3'; Alb-Cre F, 5'-CCCGCAGAACCTGAAGATG -3', Alb-Cre R, 5'GACCCGGCAAAACAGGTAG-3'. All mice were bred and maintained under specificpathogen-free conditions. All animal experiments were performed according to the National Institutes of Health Guide for the Care and Use of Laboratory Animals, with the approval by the Institutional Animal Care and Use Committee of School of Basic Medical Sciences, Shandong University (Document No. ECSBMSSDU2020-2-011). PHx was performed according to the technique described by Mitchell and Willenbring ${ }^{53}$. In brief, two-thirds of the liver (consisting of median and left lobes) were removed. The two-thirds PHx surgery operation was performed under isoflurane (Sigma-Aldrich, St. Louis, MO) anesthesia. The abdominal wall and the skin were sutured separately. For DDC-induced liver injury model, 10 -week-old mice were fed a $0.1 \%$ DDC-supplemented diet for 10 days. For the HFD model, 8-weeks-old mice were fed with HFD diet (TP2330055A, Trophic Animal Feed HighTech Co.,Ltd.) for 20 weeks.

\section{Human samples}

Human adult liver samples used in this study were obtained from five patients (males and females of 30 to 65 years old) during the hepatectomy for benign or malignant liver tumors collected from Qilu Hospital of Shandong University (Jinan, China). Human fetal liver ( $\mathrm{n}=$ 1) sample was obtained after elective pregnancy termination by dilatation and vacuum aspiration due to autosomal recessive polycystic kidney disease in the Center for Reproductive Medicine, Shandong University (Jinan, China). The tissues were collected 
within 10-15 min, placed in sterile polypropylene vials and flash-frozen in liquid nitrogen. The study was approved by the Committee of School of Basic Medical Sciences, Shandong University (Document No. ECSBMSSDU2021-1-19). All samples were stored at $-80^{\circ} \mathrm{C}$ prior to analysis.

\section{Primary hepatocyte culture}

Hepatocytes from mouse livers were isolated using a two-step enzymatic perfusion protocol ${ }^{54}$. The viability of the isolated hepatocytes was determined by trypan blue exclusion, and only preparations of over $90 \%$ viability were used. The hepatocytes were seeded onto rat tail collagen-coated tissue culture plastics in William's $\mathrm{E}$ containing $10 \%$ fetal bovine serum, left to attach for $4 \mathrm{~h}$ and then washed twice with phosphate-buffered saline (PBS) to remove unattached cells. The hepatocytes were cultured in arginine-free William's E medium supplemented with penicillin $(100 \mathrm{IU} / \mathrm{ml})$, streptomycin $(100 \mu \mathrm{g} / \mathrm{ml})$ before use.

\section{METHOD DETAILS}

\section{Measurement of serum parameters}

The liver function of animals in this study was assayed by measuring the concentrations of ALT (105-000442-00, Mindray), AST (105-000443-00, Mindray), AP (105-000444-00, Mindray), TG (105-000449-00, Mindray) and TC (105-000448-00, Mindray) in serum using a Fully Automated Biochemistry Analyze System (BS-240VET, Mindray) according to the manufacturer's instructions.

\section{Adeno-associated viral vector production}

The production of recombinant AAV8 was performed as previously described ${ }^{55}$. Briefly, HEK293T cells were seeded in a 150-mm plate and co-transfected with pAAV-TBG-Cre, pAAV2/8-RC, and pHelper in 1:1:1 molar ratio using PEI MAX 40K. $72 \mathrm{~h}$ post transfection, the supernatants were harvested and filtered with $0.22 \mu \mathrm{m}$ filter, then concentrated with Amicon Ultra-15 centrifugal filter unit, the titer of virus stock were determined by qPCR.

\section{Sirius Red Staining}

Slides were placed flat in a humid chamber, 200-300 $\mu$ of Sirius Red/Fast Green (Top0293 Biotopped) was added to cover tissues, and stained for $30 \mathrm{~min}$. Slides were thoroughly rinsed in water and briefly dehydrated through sequential alcohols, cleared briefly in xylenes, and scanned with VS120 (Olympus).

\section{Immunohistochemistry}

Mouse livers were removed, fixed overnight in $4 \%$ formalin and processed for paraffin embedding. Tissue sections were stained with hematoxylin and eosin (H\&E) using standard reagents and protocols. For IHC, slides were de-paraffinized, rehydrated, and boiled in a microwave for 10 minutes in $10 \mathrm{mM}$ citrate buffer or Tris-EDTA buffer (According to manufacturer's protocol). The slides were allowed to cool, washed three times, incubated with $4 \% \mathrm{H}_{2} \mathrm{O}_{2}$ to block endogenous peroxidase activity, washed three times, and blocked with $5 \%$ albumin bovine in $\mathrm{IHC}$ wash buffer for $30 \mathrm{~min}$. Slides were incubated with 
primary antibodies overnight at $4^{\circ} \mathrm{C}$. The next day, slides were washed three times, and incubated with horseradish peroxidase (HRP)-linked secondary antibodies for $1 \mathrm{~h}$ at room temperature. Specimens were washed three times then developed with the DAB substrate kit (ZLI-9018, ZSGB-BIO) and counterstained with hematoxylin. Antibodies used in this study are summarized in Key Resources Table.

\section{Oil Red O staining}

Liver lipid accumulation was confirmed by Modified Oil Red O stain kit (G1261, Solarbio) according to the manufacturer's instructions. In brief, frozen slices of liver $(6-10 \mu \mathrm{m})$ were fixed in $10 \%$ formaldehyde for $10 \mathrm{~min}$, and then washed with $60 \%$ isopropanol for $30 \mathrm{~s}$. Liver tissues were stained in Modified Oil Red $\mathrm{O}$ solution for $15 \mathrm{~min}$. After staining, the slices were washed with $60 \%$ isopropanol and then with $\mathrm{H}_{2} \mathrm{O}$. Images were obtained with Automated Slide Scanner (VS120, Olympus).

\section{$\mathrm{m}^{6} \mathrm{~A}$ dot blot assay}

Total RNA was extracted with Total RNA Extraction Reagent (R401-01, Vazyme Biotech co.,Itd) according to the manufacturer's instructions. RNA samples were quantified by NanoDrop 2000 (Thermo Scientific). For dot blot, $100 \mathrm{ng}$ mRNA were denatured at $95^{\circ} \mathrm{C}$ for $3 \mathrm{~min}$, followed by chilling on ice immediately. mRNA was dropped directly onto the Hybond-N+ membrane (GE Healthcare), air-dried for $10 \mathrm{~min}$ and crosslinked by ultraviolet irradiation. The membranes were washed with $0.1 \%$ TBST $(0.1 \%$ Tween-20 in $1 \times$ TBS, $\mathrm{pH} 7.4$ ) and blocked with $5 \%$ non-fat milk in $0.1 \%$ TBST. Then, the anti- ${ }^{6} \mathrm{~A}$ antibody (202003, Synaptic Systems) was diluted 1:1,000 in Primary Antibody Dilution Buffer (A1810, Solarbio) and incubated with the membranes for overnight at $4{ }^{\circ} \mathrm{C}$ with gentle shaking. The membranes were washed extensively and incubated with goat anti-Rabbit IgG-HRP (1:10,000 dilution, Proteintech) for 1 hour at room temperature. After extensive wash, the membranes were developed by enhanced chemiluminescence with Hyperfilm ECL (GE Healthcare). We also applied methylene blue staining to verify that equal amounts of RNA samples were loaded on the membrane. The intensity of dot blot signal was quantified by ImageJ.

\section{Transmission Electron Microscopy}

Mouse were put to death under deep anesthesia, and the liver tissue was removed, washed fast with PBS, immediately placed in $3 \%$ glutaraldehyde fixative solution $(\mathrm{pH} 7.4)$, the sample block trimming $1 \mathrm{~mm} \times 1 \mathrm{~mm} \times 3 \mathrm{~mm}$, according to the conventional TEM sample preparation method followed by rinsing, $1 \%$ osmic acid (OsO4) fixed, rinsing, dehydration, soaked, embedded in Epon812 (TAAB Laboratories, Berkshire, UK). Ultra-thin radial sections were cut from the basal and middle turns with lead citrate and uranyl acetate electron staining. Finally, the sections were observed using a transmission electron microscope (JEOL-1200EX).

\section{RNA decay assay}

Primary hepatocytes were seeded in 24-well plates and incubated overnight at $37^{\circ} \mathrm{C}$. The following day, actinomycin D (A1410, Sigma) was added to the cells at a final concentration 
of $10 \mu \mathrm{g} / \mathrm{ml}$. After incubation for indicated time points, cells were collected and RNA samples were extracted for qPCR to determine the Smpd3, Smpd1, Sptlc1 and Sptlc2 $m R N A$ levels. The data were normalized to the $t=0$ time point. For $Y$ thdf2-mediated decay assay, we first transfected the primary hepatocytes with control or two independent Ythdf2 siRNAs, then performed the actinomycin $D$ treatment.

\section{RNA-seq and MeRIP-seq}

Total RNA was extracted from WT and Mettl $3^{\triangle \text { hep }}$ livers with Total RNA Extraction Reagent (R401-01, Vazyme Biotech co.,Itd) following the manufacturer's instructions, and samples were quantified using a NanoDrop ND-1000 instrument. The RNA libraries were prepared with NEBNext Ultra II Directional RNA Library Prep Kit for Illumina (NEB) according to the manufacturer's instructions. Three independent biological replicates for each group were performed for RNA-seq. Sequencing reads were trimmed using StringTie and mapped to the mouse genome database $(\mathrm{GRCm} 38)$ with Hisat2 software. Stringtie was used to calculate the TPM of each gene to represent their mRNA expression level. The differential genes were identified by using the limma package. For MeRIP-seq, intact mRNA was isolated from total RNA samples and chemically fragmented to 100-nucleotide-long fragments. MeRIP was performed to enrich $\mathrm{m}^{6} \mathrm{~A}$-methylated $\mathrm{mRNAs}$ using an anti- $\mathrm{m}^{6} \mathrm{~A}$ antibody (202003, Synaptic Systems). The TruSeq Stranded mRNA Library Preparation kit (Illumina) was used for library preparation of both $\mathrm{m}^{6} \mathrm{~A}$-enriched RNAs and input mRNAs. The libraries were subjected to denaturation to obtain single-stranded DNA molecules and captured on Illumina flow cells. Then, they were amplified in situ as sequencing clusters and sequenced for 150 cycles on an Illumina HiSeq 4000 system as per the manufacturer's instructions. The image analysis and base calling were carried out using Solexa pipeline v.1.8 (Off-Line Base Caller software, v.1.8). The sequencing quality was examined by FastQC software, and trimmed reads (pass Illumina quality filter, trimmed adaptor bases by cutadapt) were aligned to genome sequences from Ensembl using Hisat2software (v.2.1.0). The aligned reads were used for peak calling by exomePeak, and statistically significant MeRIP-enriched regions (peaks) were identified for each transcript and compared by exomePeak. The MeRIP-enriched regions (peaks) were annotated using the overlapped gene with the newest version of the Ensembl database. Then, statistical analysis of the $\mathrm{m}^{6} \mathrm{~A}$ peak in each transcript region was done. Three independent biological replicates for each group were performed for MeRIP-seq.

\section{$m^{6}$ A-RIP qRT-PCR}

Total RNA from Mettl $3^{\Delta \text { hep }}$ and WT liver tissues were extracted with Total RNA Extraction Reagent (R401-01, Vazyme Biotech co.,Itd) following the manufacturer's instructions. RNA fragmentation and immunoprecipitated of $\mathrm{m}^{6} \mathrm{~A}$-containing RNA fragments were performed as previous protocol ${ }^{56}$. Briefly, RNA was fragmented into $\sim 100 \mathrm{nt}$ and incubated with anti$\mathrm{m}^{6} \mathrm{~A}$ antibody (202003, Synaptic System) or rabbit IgG (CST) for 2 hours at $4{ }^{\circ} \mathrm{C}$, then were immunoprecipitated by incubation with Protein A beads (Thermo Fisher Scientific) for 2 hours at $4{ }^{\circ} \mathrm{C}$. Captured RNA was competitively purified by ethanol precipitation. For qRT$P C R$, reverse transcription and $\mathrm{QPCR}$ were performed with HiScript II Q Select RT SuperMix for qPCR (+gDNA wiper) (R233-01, Vazyme Biotech co.,Itd) and the real-time 
PCR analysis was performed with Universal SYBR Green Fast qPCR Mix (RK21203, Abclonal) by CFX Connect Real-Time PCR Detection System (BIO-RAD). The amount of target was calculated by $\triangle \Delta \mathrm{Ct}$ method and results were presented relative to these obtained with Input. Gapdh was used as negative control ${ }^{57}$.

\section{Western blot analysis.}

Total protein was isolated from tissue samples using RIPA lysis buffer $(50 \mathrm{mM}$ Tris- $\mathrm{HCl}, \mathrm{pH}$ 7.4, $150 \mathrm{mM} \mathrm{NaCl}, 1 \% \mathrm{NP}-40,0.5 \%$ sodium deoxycholate, $0.1 \%$ SDS, $1 \mathrm{mM}$ EDTA) with protease inhibitor cocktail tablets (HY-K0011; MedChemExpress) and phosphatase inhibitor tablets (G2007, Servicebio). The total protein samples were loaded and separated on SDS-PAGE gels and transferred to PVDF membranes (IPVH00010; Merck Millipore). The membranes were blocked with $5 \%$ skim milk and incubated with the indicated primary antibodies overnight at $4{ }^{\circ} \mathrm{C}$, followed by incubation with the corresponding secondary antibodies for $1 \mathrm{~h}$ at room temperature. The membranes were visualized by enhanced chemiluminescence (ECL) reagents (E411-03; Vazyme) and captured by a Chemiluminescence Imaging System (Tanon 5500). Gapdh was used as a loading control. Antibodies used in this study are summarized in Key Resources Table.

\section{Quantitative real-time PCR (qRT-PCR)}

Total RNA was extracted with Total RNA Extraction Reagent (R401-01, Vazyme Biotech co.,Itd), then $1 \mu \mathrm{g}$ RNA was reverse transcribed into cDNA using HiScript II Q RT SuperMix for qPCR (+gDNA wiper) (R223-01, Vazyme) following the manufacturer's instructions. The real-time PCR analysis was performed with Universal SYBR Green Fast qPCR Mix (RK21203, Abclonal) by CFX Connect Real-Time PCR Detection System (BIO-RAD). The relative RNA expression level was normalized to Gapdh according to the $\triangle \Delta \mathrm{Ct}$ calculation method. The primers used in this study are listed in Supplementary Table 4.

\section{Mitochondrial membrane potential assay}

Primary hepatocytes were seeded in 24-well plates. $0.5 \mathrm{~mL} \mathrm{JC}-1$ working solution was added in the medium and incubated in $\mathrm{CO}_{2}$ incubator for $20 \mathrm{~min}$. The staining solution was removed and cells were washed with PBS twice. Images were collected with Axio Vert.A1 inverted fluorescence microscope (Zeiss).

\section{Untargeted lipidomic analysis}

Six independent biological replicates from each group were performed for untargeted lipidomic analysis. Liver tissue sample was thawed on ice. $50 \mathrm{mg}$ of each tissue sample were subjected to liquid extraction. Ultra performance liquid chromatography (UPLC) and tandem mass spectrometry (MS/MS) analysis was performed at Bgi Genomics Co., Ltd. (Shenzhen, China).

\section{Targeted Lipidomic analysis}

Targeted lipidomic analysis was performed on a liquid chromatography with tandem mass spectrometry (LC-MS/MS 8060, Shimadzu Corporation, Kyoto, Japan) equipped with an electrospray ionization (ESI) source. Liver tissue sample was thawed on ice. Take $20 \mathrm{mg}$ 
of one sample and homogenize it with $1 \mathrm{~mL}$ mixture (include methanol, MTBE and internal standard mixture) and steel ball. Take out the steel ball and whirl the mixture for $15 \mathrm{~min}$. Add $200 \mu \mathrm{L}$ of water and whirl the mixture for $1 \mathrm{~min}$, and then centrifuge it with 12,000 rpm at $4^{\circ} \mathrm{C}$ for $10 \mathrm{~min}$. Extract $300 \mu \mathrm{L}$ supernatant and concentrate it. Dissolve powder with 200 $\mu \mathrm{L}$ mobile phase $\mathrm{B}$, then stored in $-80^{\circ} \mathrm{C}$. Finally take the dissolving solution into the sample bottle for LC-MS/MS analysis. The sample extracts were analyzed using an LC-ESIMS/MS system (UPLC, ExionLC AD, https://sciex.com.cn/; MS, QTRAP® 6500+ System, https://sciex.com/). The analytical conditions were as follows, UPLC: column, Thermo Accucore ${ }^{\mathrm{TM}} \mathrm{C} 30(2.6 \mu \mathrm{m}, 2.1 \mathrm{~mm} \times 100 \mathrm{~mm}$ i.d. $)$; solvent system, A: acetonitrile/water (60/40,V/V , 0.1\% formic acid, $10 \mathrm{mmol} / \mathrm{L}$ ammonium formate), B: acetonitrile/isopropanol (10/90 VV/N, $0.1 \%$ formic acid, $10 \mathrm{mmol} / \mathrm{L}$ ammonium formate); gradient program, $A / B$ (80:20, V/V) at $0 \mathrm{~min}, 70: 30 \mathrm{~V} / \mathrm{V}$ at $2.0 \mathrm{~min}, 40: 60 \mathrm{~V} / \mathrm{V}$ at $4 \mathrm{~min}, 15: 85 \mathrm{~V} / \mathrm{V}$ at $9 \mathrm{~min}, 10: 90$ $\mathrm{V} / \mathrm{V}$ at $14 \mathrm{~min}, 5: 95 \mathrm{~V} / \mathrm{V}$ at $15.5 \mathrm{~min}, 5: 95 \mathrm{~V} / \mathrm{V}$ at $17.3 \mathrm{~min}, 80: 20 \mathrm{~V} / \mathrm{V}$ at $17.3 \mathrm{~min}, 80: 20$ $\mathrm{V} / \mathrm{V}$ at $20 \mathrm{~min}$; flow rate, $0.35 \mathrm{ml} / \mathrm{min}$; temperature, $45^{\circ} \mathrm{C}$; injection volume: $2 \mu \mathrm{L}$. The effluent was alternatively connected to an ESI-triple quadrupole-linear ion trap (QTRAP)MS. LIT and triple quadrupole (QQQ) scans were acquired on a triple quadrupole-linear ion trap mass spectrometer (QTRAP), QTRAP® 6500+ LC-MS/MS System, equipped with an ESI Turbo lon-Spray interface, operating in positive and negative ion mode and controlled by Analyst 1.6.3 software (Sciex). The ESI source operation parameters were as follows: ion source, turbo spray; source temperature $500^{\circ} \mathrm{C}$; ion spray voltage (IS) 5500 V (Positive), $-4500 \mathrm{~V}$ (Neagtive); lon source gas 1 (GS1), gas 2 (GS2), curtain gas (CUR) were set at 45,55 , and $35 \mathrm{psi}$, respectively. Instrument tuning and mass calibration were performed with 10 and $100 \mu \mathrm{mol} / \mathrm{L}$ polypropylene glycol solutions in QQQ and LIT modes, respectively. QQQ scans were acquired as MRM experiments with collision gas (nitrogen) set to 5 psi. DP and CE for individual MRM transitions was done with further DP and CE optimization. A specific set of MRM transitions were monitored for each period according to the metabolites eluted within this period. Four independent biological replicates from each group were performed for targeted lipidomic analysis. 
629 This study was supported by National Key Research and Development Program of China 630 to S.L. (2020YFA0804400); National Natural Science Foundation of China (81802799, 631 82071854, 32100590); Shandong Provincial Natural Science Foundation, China 632 (ZR2019BH002, ZR2020QH038); Jiangsu Provincial Natural Science Foundation, China 633 (BK20180222); Funds for Youth Interdisciplinary and Innovation Research Groups of 634 Shandong University (2020QNQT003 and 2020QNQT009); and Taishan Scholars 635 Program of Shandong Province. M.H. was supported by an ERC consolidator grant and 636 the Rainer Hoenig Stiftung. The authors thank Sexin Huang of the Center for Reproductive 637 Medicine, Shandong University for kindly providing fetal liver sample. The authors thank 638 the technical assistance at the Core facility of Advanced Medical Research Institute of 639 Shandong university.

\section{AUTHOR CONTRIBUTIONS}

641 D.Y., M.H. Y.X. and S.L. designed the experiments, interpreted the data, and wrote the 642 manuscript. S.W. and S.C. contributed to the experimental design and performed in vivo 643 animal studies. S.W., P.H. and B.X. performed in vitro experiments. Y.Z. and Z.X. 644 performed AAV8 packaging. P.Z., P.M. and C.Z. contributed to histological analysis. All the 645 authors have approved the final version of the manuscript for publication.

\section{DECLARATION OF INTERESTS}

647 All authors declared no competing interests. 
650 1. Plagge, A., et al. The imprinted signaling protein $X L$ alpha $s$ is required for postnatal 651 adaptation to feeding. Nat Genet 36, 818-826 (2004).

652 2. Uddin, M., et al. Distinct genomic signatures of adaptation in pre- and postnatal 653 environments during human evolution. Proc Natl Acad Sci U S A 105, 3215-3220 (2008).

654 3. Zamboulis, D.E., et al. Postnatal mechanical loading drives adaptation of tissues primarily 655 through modulation of the non-collagenous matrix. Elife $\mathbf{9}$ (2020).

6564 . Reese, J., et al. Coordinated regulation of fetal and maternal prostaglandins directs 657 successful birth and postnatal adaptation in the mouse. Proc Natl Acad Sci U S A 97, 9759$6589764(2000)$.

659 5. Willers, M., et al. S100A8 and S100A9 Are Important for Postnatal Development of Gut 660 Microbiota and Immune System in Mice and Infants. Gastroenterology 159, 2130-2145 $661 \quad$ e2135 (2020).

662 6. Rossant, J., Vijh, K.M., Grossi, C.E. \& Cooper, M.D. Clonal origin of haematopoietic colonies 663 in the postnatal mouse liver. Nature 319, 507-511 (1986).

6647 Lotto, J., et al. Single-Cell Transcriptomics Reveals Early Emergence of Liver Parenchymal 665

8. Gunewardena, S.S., et al. Deciphering the Developmental Dynamics of the Mouse Liver 667 668 Transcriptome. PLoS One 10, e0141220 (2015).

671 $\mathrm{Li}, \mathrm{T}$., et al. Multi-stage analysis of gene expression and transcription regulation in C57/B6 mouse liver development. Genomics 93, 235-242 (2009).

13. Mu, T., et al. Embryonic liver developmental trajectory revealed by single-cell RNA

14. Gordillo, M., Evans, T. \& Gouon-Evans, V. Orchestrating liver development. Development 142, 2094-2108 (2015).

680

15. Frye, M., Harada, B.T., Behm, M. \& He, C. RNA modifications modulate gene expression during development. Science 361, 1346-1349 (2018).

16. Dong, L., et al. The loss of RNA N(6)-adenosine methyltransferase Mettl14 in tumorassociated macrophages promotes CD8(+) T cell dysfunction and tumor growth. Cancer Cel/39, 945-957 e910 (2021).

17. He, P.C. \& He, C. m(6) A RNA methylation: from mechanisms to therapeutic potential.

687 18. Tong, J., Flavell, R.A. \& Li, H.B. RNA m(6)A modification and its function in diseases. Front Med 12, 481-489 (2018).

689 19. Wang, Y., et al. METTL3 is essential for postnatal development of brown adipose tissue $690 \quad$ and energy expenditure in mice. Nat Commun 11, 1648 (2020).

691 20. Li, H.B., et al. m(6)A mRNA methylation controls T cell homeostasis by targeting the IL692 7/STAT5/SOCS pathways. Nature 548, 338-342 (2017). 
21. Shi, H.L., Wei, J.B. \& He, C. Where, When, and How: Context-Dependent Functions of RNA Methylation Writers, Readers, and Erasers. Mol Ce// 74, 640-650 (2019).

22. Wang, C.X., et al. METTL3-mediated m6A modification is required for cerebellar development. PLOS Bio/ 16, e2004880 (2018).

23. Yao, Y., et al. METTL3-dependent m(6)A modification programs $\mathrm{T}$ follicular helper cell differentiation. Nat Commun 12, 1333 (2021).

24. Kyrmizi, I., et al. Plasticity and expanding complexity of the hepatic transcription factor network during liver development. Genes Dev 20, 2293-2305 (2006).

25. Karagianni, P., Moulos, P., Schmidt, D., Odom, D.T. \& Talianidis, I. Bookmarking by Nonpioneer Transcription Factors during Liver Development Establishes Competence for Future Gene Activation. Cell Rep 30, 1319-1328 e1316 (2020).

26. Batista, P.J., et al. m(6)A RNA modification controls cell fate transition in mammalian embryonic stem cells. Cell Stem Cel/ 15, 707-719 (2014).

27. Zhang, C., et al. m(6)A modulates haematopoietic stem and progenitor cell specification. Nature 549, 273-276 (2017).

28. Postic, C., et al. Dual roles for glucokinase in glucose homeostasis as determined by liver and pancreatic beta cell-specific gene knock-outs using Cre recombinase. $J$ Biol Chem 274, 305-315 (1999).

29. Li, Y., et al. m(6)A Regulates Liver Metabolic Disorders and Hepatogenous Diabetes. Genomics Proteomics Bioinformatics 18, 371-383 (2020).

30. Xie, W., Ma, L.L., Xu, Y.Q., Wang, B.H. \& Li, S.M. METTL3 inhibits hepatic insulin sensitivity via N6-methyladenosine modification of Fasn mRNA and promoting fatty acid metabolism. Biochem Biophys Res Commun 518, 120-126 (2019).

31. Green, C.D., Maceyka, M., Cowart, L.A. \& Spiegel, S. Sphingolipids in metabolic disease: The good, the bad, and the unknown. Cell Metab 33, 1293-1306 (2021).

32. Deng, $X$., et al. Ceramide biogenesis is required for radiation-induced apoptosis in the germ line of C. elegans. Science 322, 110-115 (2008).

33. Turpin, S.M., et al. Obesity-induced CerS6-dependent C16:0 ceramide production promotes weight gain and glucose intolerance. Cell Metab 20, 678-686 (2014).

34. Wang, $\mathrm{X}$., et al. N-6-methyladenosine-dependent regulation of messenger RNA stability. Nature 505, 117-+ (2014).

35. Zaccara, S. \& Jaffrey, S.R. A Unified Model for the Function of YTHDF Proteins in Regulating m(6)A-Modified mRNA. Ce// 181, 1582-+ (2020).

36. Lasman, L., et al. Context-dependent functional compensation between Ythdf $m(6) A$ reader proteins. Gene Dev 34, 1373-1391 (2020).

37. Moylan, J.S., et al. Neutral sphingomyelinase-3 mediates TNF-stimulated oxidant activity in skeletal muscle. Redox Bio/2, 910-920 (2014).

38. Heinrich, M., et al. Cathepsin D links TNF-induced acid sphingomyelinase to Bid-mediated caspase- 9 and -3 activation. Cell Death Differ 11, 550-563 (2004).

39. Ogura, Y., et al. Postnatal changes in gene expression of retinal dehydrogenase and retinoid receptors in liver of rats. Life Sci 74, 1519-1528 (2004).

40. Zhong, X., et al. Circadian Clock Regulation of Hepatic Lipid Metabolism by Modulation of m(6)A mRNA Methylation. Cell Rep 25, 1816-1828 e1814 (2018).

41. Barajas, J.M., et al. METTL3 Regulates Liver Homeostasis, Hepatocyte Ploidy, and Circadian 
Rhythm-Controlled Gene Expression in Mice. Am J Pathol (2021).

42. Ji, R.P., et al. Increased de novo ceramide synthesis and accumulation in failing myocardium. Jci Insight 2(2017).

43. Reforgiato, M.R., et al. Inhibition of ceramide de novo synthesis as a postischemic strategy to reduce myocardial reperfusion injury. Basic Res Cardio/111(2016).

44. Yu, Z.F., et al. Pivotal role for acidic sphingomyelinase in cerebral ischemia-induced ceramide and cytokine production, and neuronal apoptosis. J Mol Neurosci 15, 85-97 (2000).

45. Chudakova, D.A., et al. Integrin-associated Lyn Kinase Promotes Cell Survival by Suppressing Acid Sphingomyelinase Activity. J Bio/ Chem 283, 28806-28816 (2008).

46. Tuzcu, H., et al. Neutral sphingomyelinase inhibition alleviates apoptosis, but not ER stress, in liver ischemia-reperfusion injury. Free Radical Res 51, 253-268 (2017).

47. Zager, R.A., Iwata, M., Conrad, D.S., Burkhart, K.M. \& Igarashi, Y. Altered ceramide and sphingosine expression during the induction phase of ischemic acute renal failure. Kidney Int 52, 60-70 (1997).

48. Cuzzocrea, S., et al. Anti-inflammatory and anti-apoptotic effects of fumonisin B1, an inhibitor of ceramide synthase, in a rodent model of splanchnic ischemia and reperfusion injury. J Pharmacol Exp Ther 327, 45-57 (2008).

49. He, X.X. \& Schuchman, E.H. Ceramide and Ischemia/Reperfusion Injury. J Lipids 2018(2018).

50. Holland, W.L., et al. Lipid-induced insulin resistance mediated by the proinflammatory receptor TLR4 requires saturated fatty acid-induced ceramide biosynthesis in mice. Journal of Clinical Investigation 121, 1858-1870 (2011).

51. Holland, W.L., et al. An FGF21-Adiponectin-Ceramide Axis Controls Energy Expenditure and Insulin Action in Mice. Cell Metab 17, 790-797 (2013).

52. Jiang, C.T., et al. Intestinal farnesoid $X$ receptor signaling promotes nonalcoholic fatty liver disease. Journal of Clinical Investigation 125, 386-402 (2015).

53. Mitchell, C. \& Willenbring, H. A reproducible and well-tolerated method for $2 / 3$ partial hepatectomy in mice. Nat Protoc 3, 1167-1170 (2008).

54. Li, W.C., Ralphs, K.L. \& Tosh, D. Isolation and culture of adult mouse hepatocytes. Methods Mol Bio/ 633, 185-196 (2010).

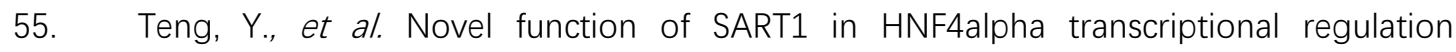
contributes to its antiviral role during HBV infection. J Hepatol (2021).

56. Dominissini, D., Moshitch-Moshkovitz, S., Salmon-Divon, M., Amariglio, N. \& Rechavi, G. Transcriptome-wide mapping of $\mathrm{N}(6)$-methyladenosine by $\mathrm{m}(6) \mathrm{A}$-seq based on immunocapturing and massively parallel sequencing. Nat Protoc 8, 176-189 (2013).

57. Liu, Y., et al. N (6)-methyladenosine RNA modification-mediated cellular metabolism rewiring inhibits viral replication. Science 365, 1171-1176 (2019). 


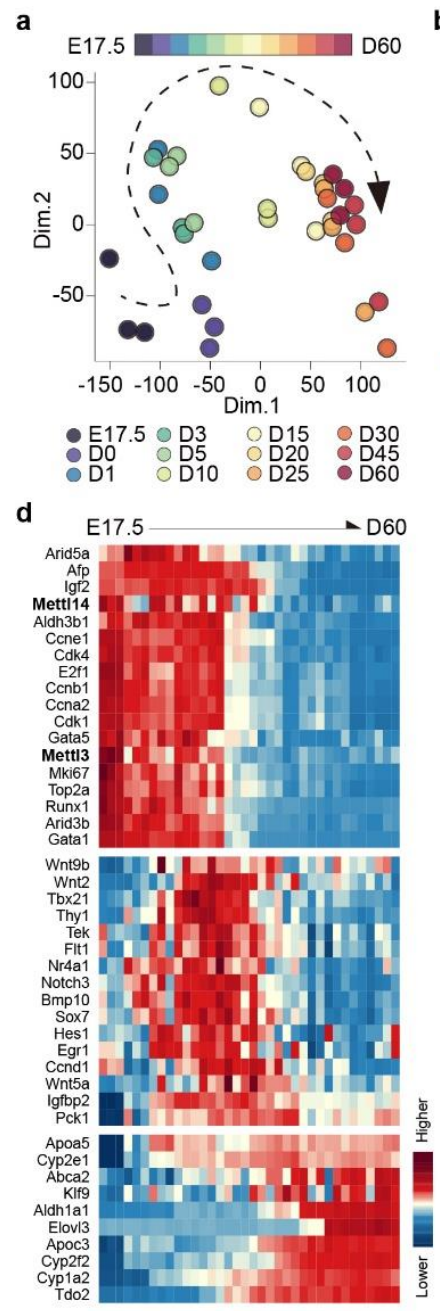

b Downregulating genes
Upregulating genes Neonatal-enriched

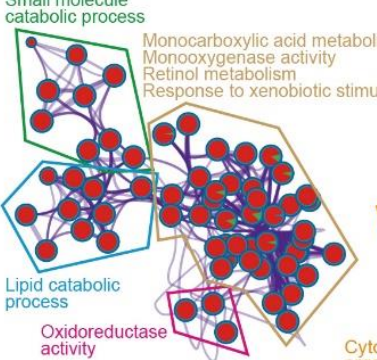

$$
\begin{aligned}
& \text { Oxidore } \\
& \text { activity }
\end{aligned}
$$

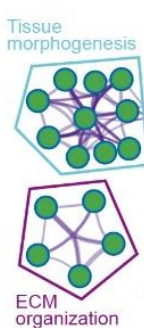
Blood vesse!
development

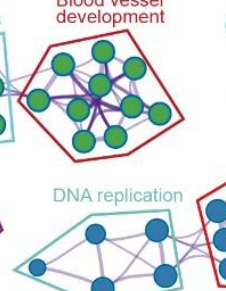

e

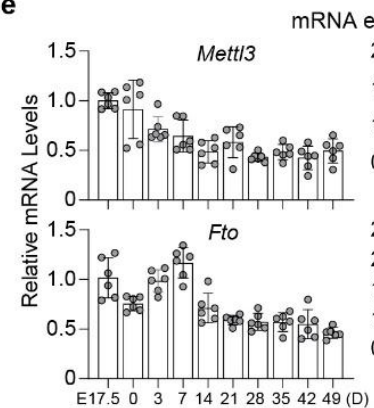

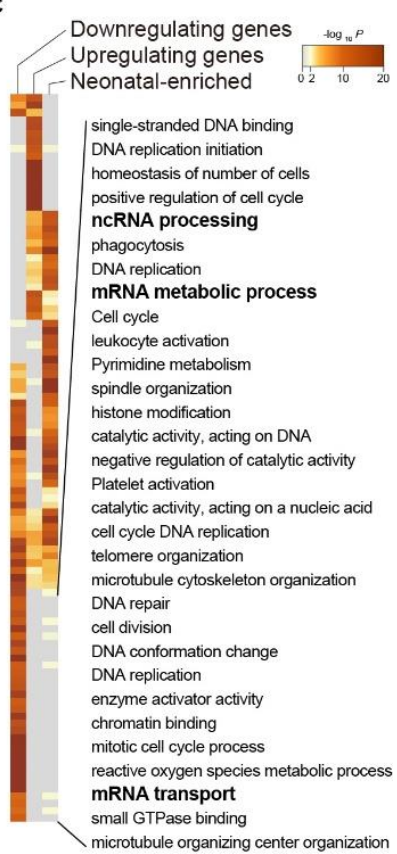
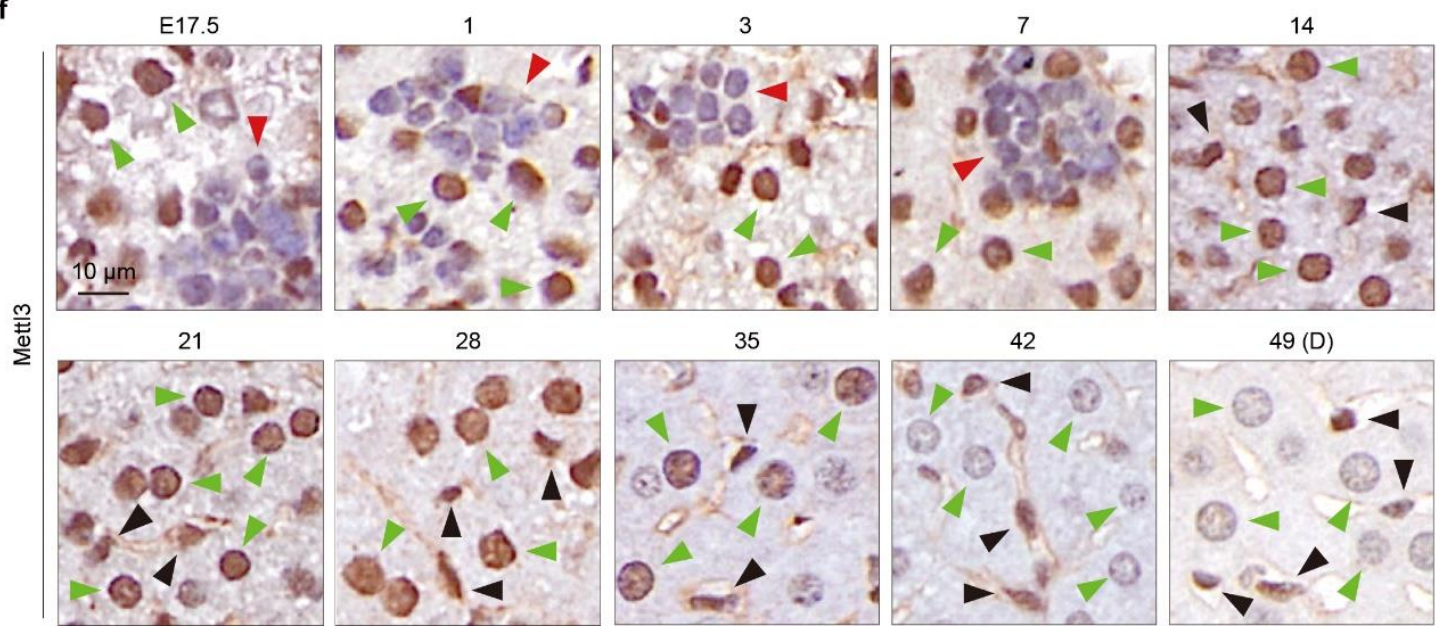

28
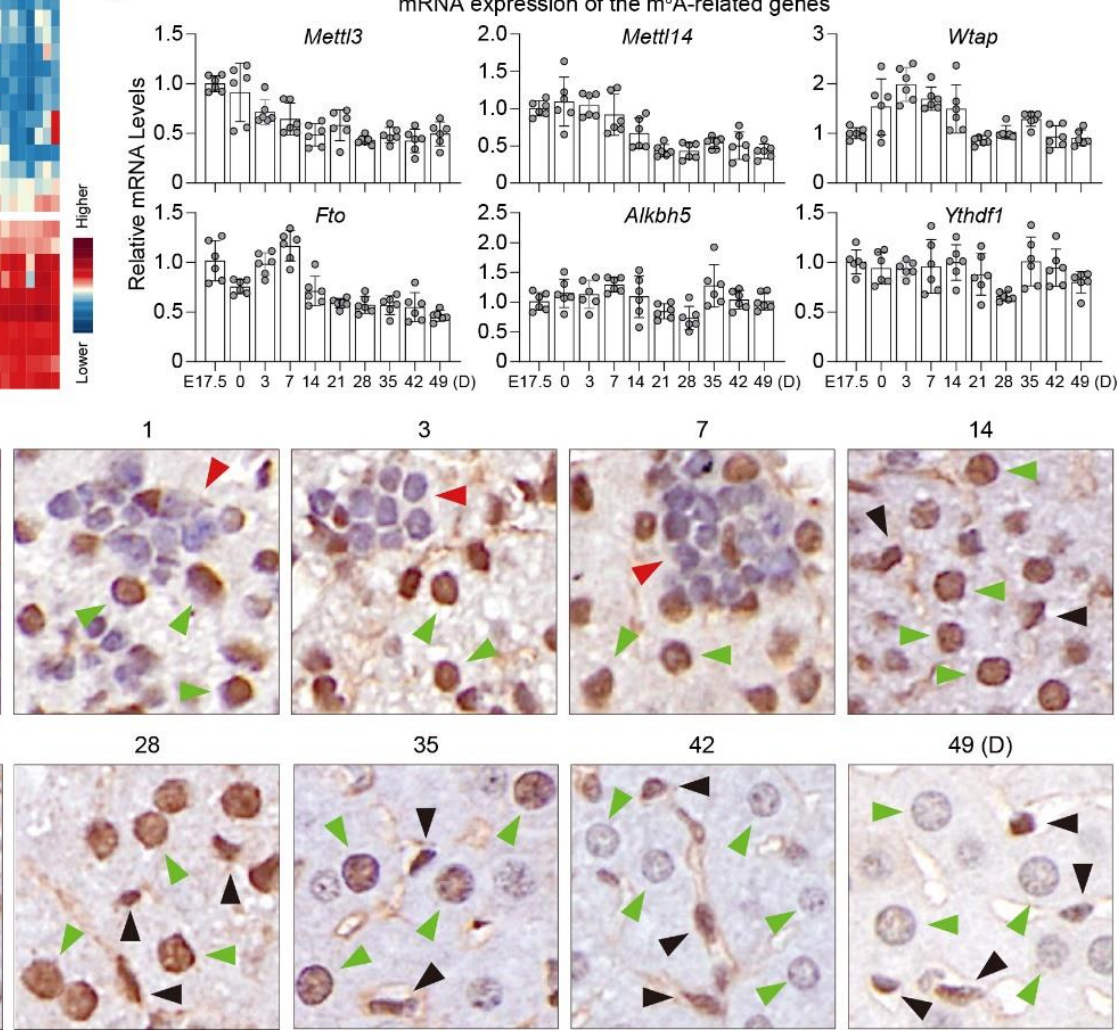

Fig. 1: Mettl3 is highly expressed in embryonic and neonatal livers and declines during postnatal liver development. a, PCA plots showing an overview of the sample distribution for transcriptomic analysis of liver samples at 12 ages. b, Functional annotation and network of enriched terms represented as pie charts colored by the identities of the three groups detected by the time-course analysis in Extended Data Fig. 1b. c, Heat map of enriched GO and KEGG terms colored by p-values (See also Extended Data Fig. 1c for the fully annotated heat map). d, Heat maps generated using 
the mRNA expression of the indicated genes in the three groups detected by the timecourse analysis. e, qRT-PCR analysis was performed to examine the mRNA expression for indicated genes in livers obtained from mice at a series of ages. f, Mett|3 IHC in liver tissues from mice at the indicated ages. The green arrowheads indicate hepatocytes, the red arrowheads indicate hematopoietic cells, and the black arrows indicate liver nonparenchymal cells. Scale bars represent $10 \mu \mathrm{m}$. 


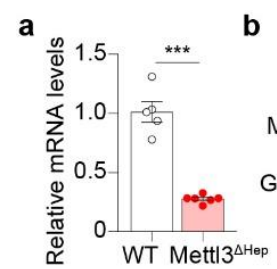

b

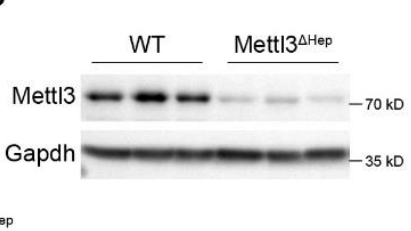

d

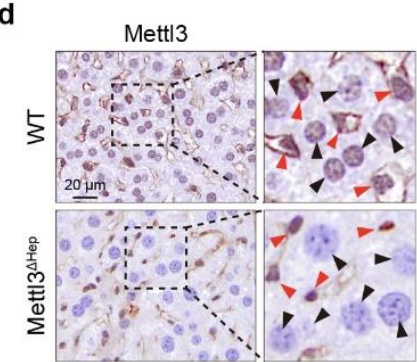

h
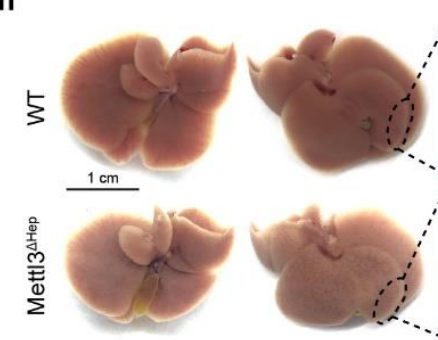

e

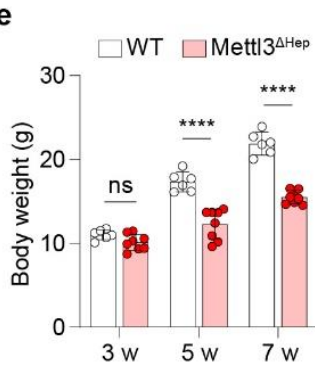

$f$

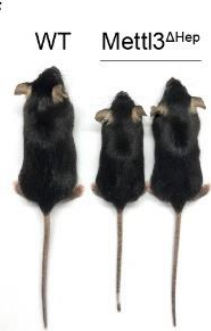

i$$
\text { 5 }
$$

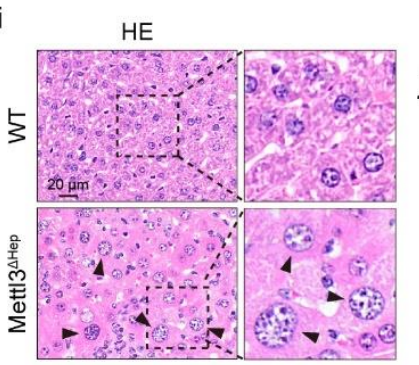

k
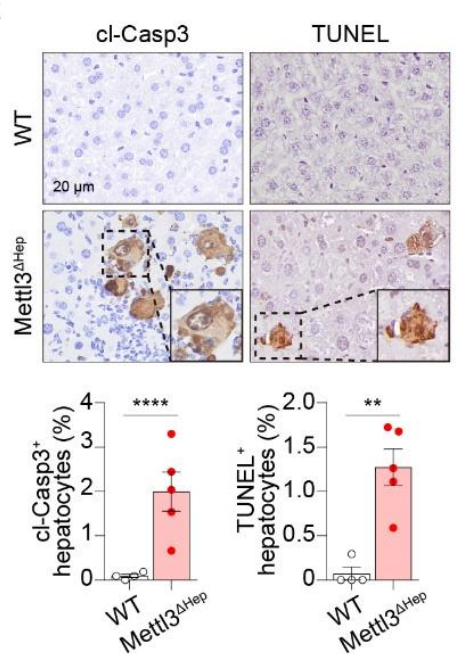

m

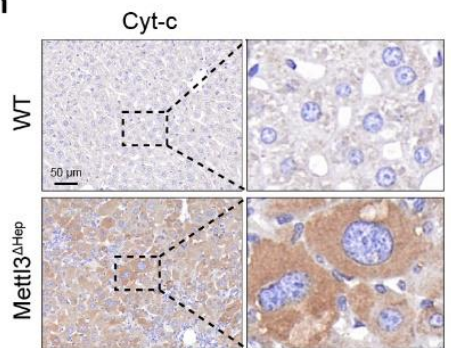

I

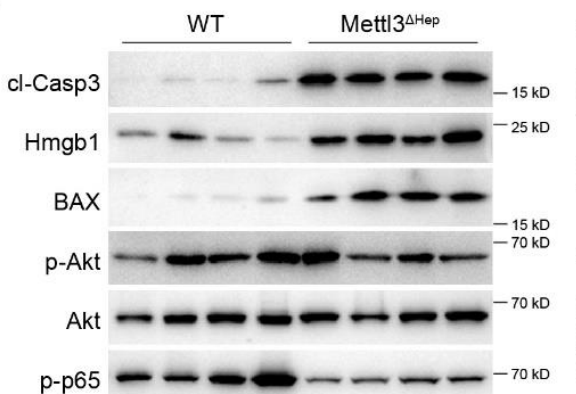

C

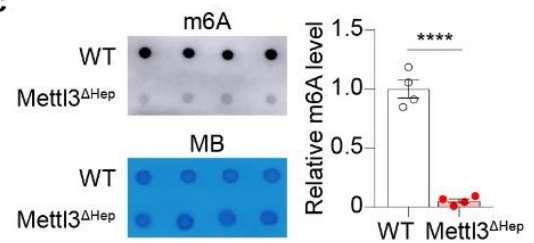

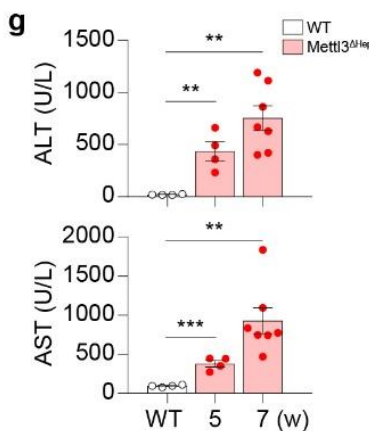

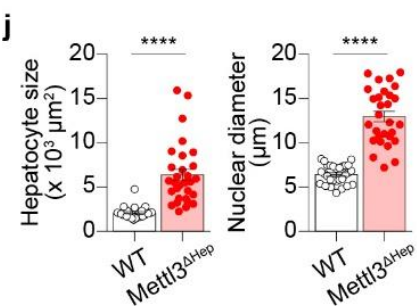

Fig. 2: Hepatic Mettl3 deficiency induces hepatocyte hypertrophy, liver injury and growth retardation during postnatal development. a, qRT-PCR for Mett/3 in WT and Mett $3^{\triangle \text { hep }}$ livers. b, Immunoblotting and quantification for Mettl3 showing the Knock-out effect in Mettl $3^{\Delta \text { hep }}$ livers. c, Dot blot and quantification of $\mathrm{m}^{6} \mathrm{~A}$ levels in WT and Mett $3^{\Delta \text { hep }}$ livers. MB, methylene blue staining. d, IHC of Mettl3 in livers from WT and Mettl $3^{\Delta \text { hep }}$ mice. 
Black arrowheads, hepatocytes; Red arrowheads, non-parenchymal cells. Scale bar, 20 $\mu \mathrm{m}$. e, Body weight of WT and Mettl $3^{\mathrm{Ahep}}$ mice at the indicated ages. f, Five-week-old Mettl3 ${ }^{\triangle h e p}$ mice and WT littermate. Scale bar, $5 \mathrm{~cm}$. g, Serum ALT and AST in WT and Mettl $3^{\Delta \text { hep }}$ mice at the indicated ages. $\mathbf{h}$, Gross appearance of livers in WT and Mettl $3^{\Delta \text { hep }}$ mice at 42 days after birth. The scale bar represents $1 \mathrm{~cm}$. i, Representative H\&E staining of livers from WT and Mettl $3^{\Delta \text { hep }}$ mice. Scale bar, $20 \mu \mathrm{m}$. j, Quantification of cell size and nuclear diameter of the hepatocytes in WT and Mettl $3^{\triangle \text { hep }}$ mice. $\mathbf{k}$, IHC and quantification for clCasp3 and TUNEL in livers from WT and Mettl $3^{\text {Ahep }}$ mice. Scale bar, $20 \mu \mathrm{m}$. I, Immunoblotting and quantification for the indicated proteins in WT and Mettl $3^{\Delta \text { hep }}$ liver lysates. m, IHC of Cyt-c in WT and Mettl3 ${ }^{\Delta \text { hep }}$ livers. Scale bar, $50 \mu \mathrm{m}$. n, IHC of Ki67, CK19 and EpCAM in livers from WT and Mettl|3 ${ }^{\Delta \text { hep }}$ mice, and quantification for Ki67 staining. Scale bar, $20 \mu \mathrm{m}$. Data are shown in mean \pm SEM; ns, not significant, ${ }^{* *} p<0.01$, ${ }^{* * *} p<0.001,{ }^{* * * *} p<0.0001$ by Student's t test. 
a

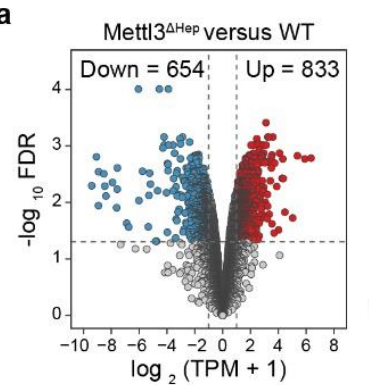

d

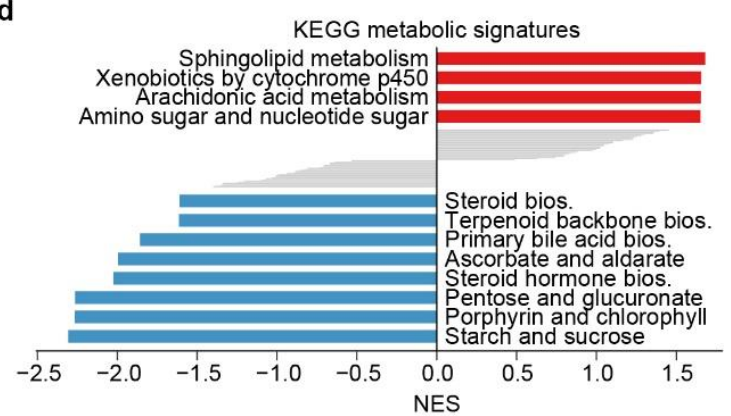

b

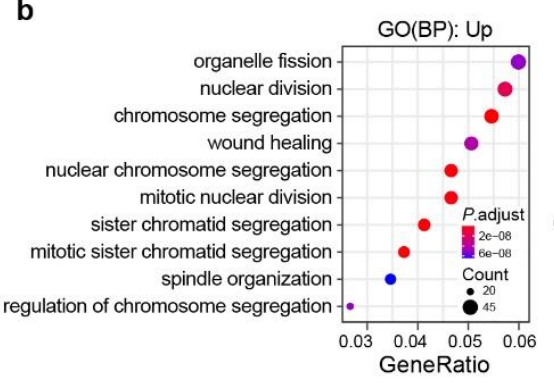

e

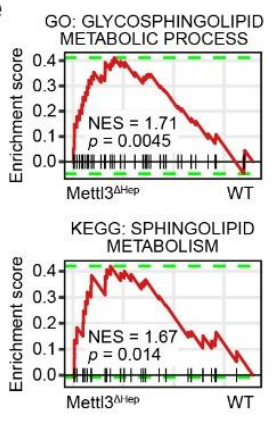

C

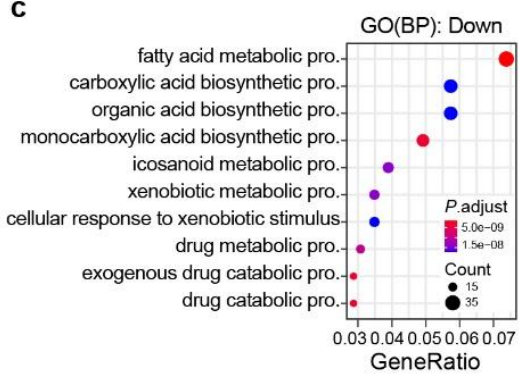

g
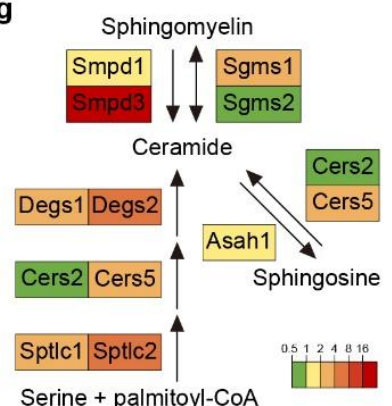

h f
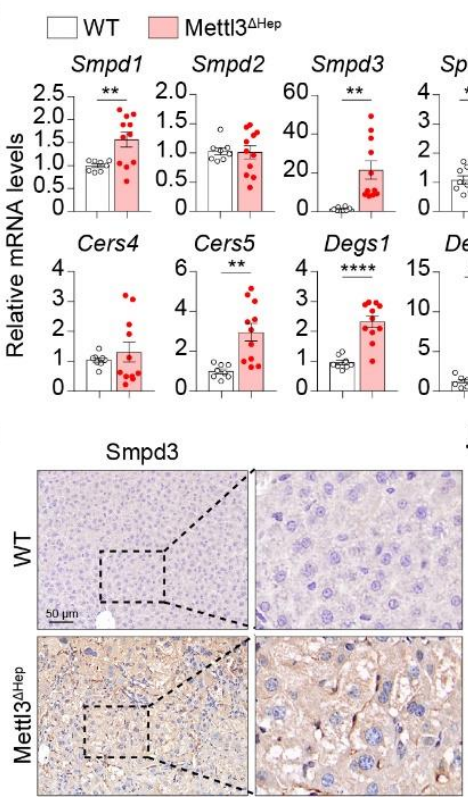

j

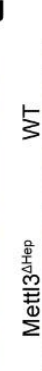

Smpd1

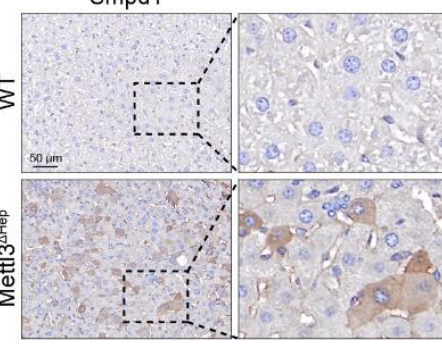

WT

Mett|3 $3^{\Delta \text { Hep }}$
811

Fig. 3: Mett|3 deficiency leads to sphingolipid metabolic reprogramming in Mettl $3^{\Delta}$ hep liver. a, Volcano plot showing the overall change of genes in Mettl $3^{\triangle \mathrm{hep}}$ liver compared to control. b,c, GO(BP) analysis of upregulated (b) and downregulated (c) genes in Mett|3 $3^{\Delta \text { hep }}$ livers over WT controls. d, Enrichment of KEGG metabolic signatures in Mett|3 $3^{\Delta h e p}$ versus WT livers. Signatures that are significantly upregulated (red) or downregulated (blue) are highlighted. e, GSEA analysis in Mettl3 ${ }^{\Delta \text { hep }}$ versus WT livers for the indicated gene sets. f, qRT-PCR detecting sphingolipid metabolism-related genes in Mett $3^{\triangle \text { hep }}$ and WT livers. $\mathbf{g}$, Schematic of sphingolipid metabolic pathways colored by expression changes in (f). $\mathbf{h}$, Immunoblotting and quantification for the indicated proteins in WT and Mettl $3^{\text {Shep }}$ liver lysates. i, j, k, Representative IHC of Smpd3 (i), Smpd1 (j) and Sptlc2 (k) in WT and Mettl $3^{\Delta \text { hep }}$ livers. Scale bar, $50 \mu \mathrm{m}$. Data are shown in mean \pm SEM; ${ }^{*} p<0.05,{ }^{* *} p<0.01,{ }^{* * *} p<0.001,{ }^{* * *} p<0.0001$ by Student's t test. 
a

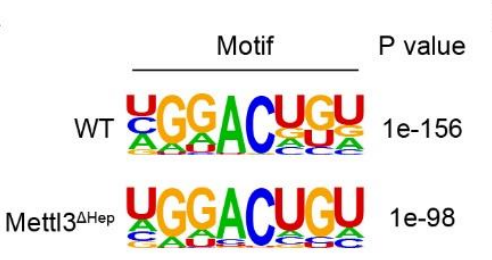

b 1.5

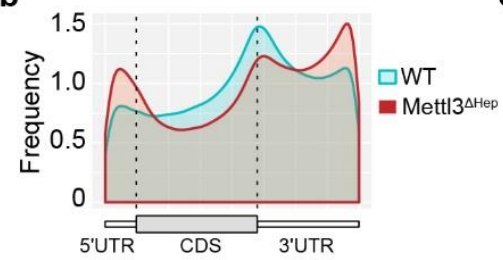

C

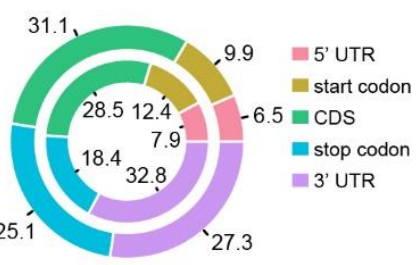

d
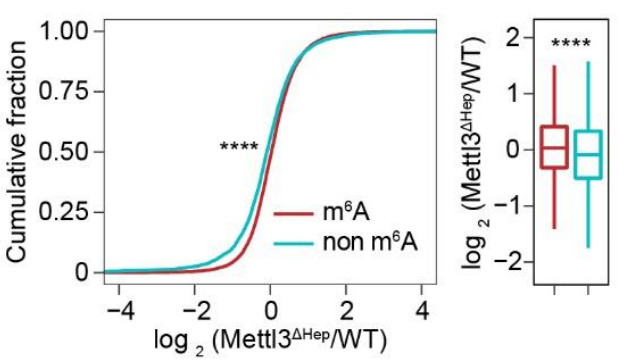

e

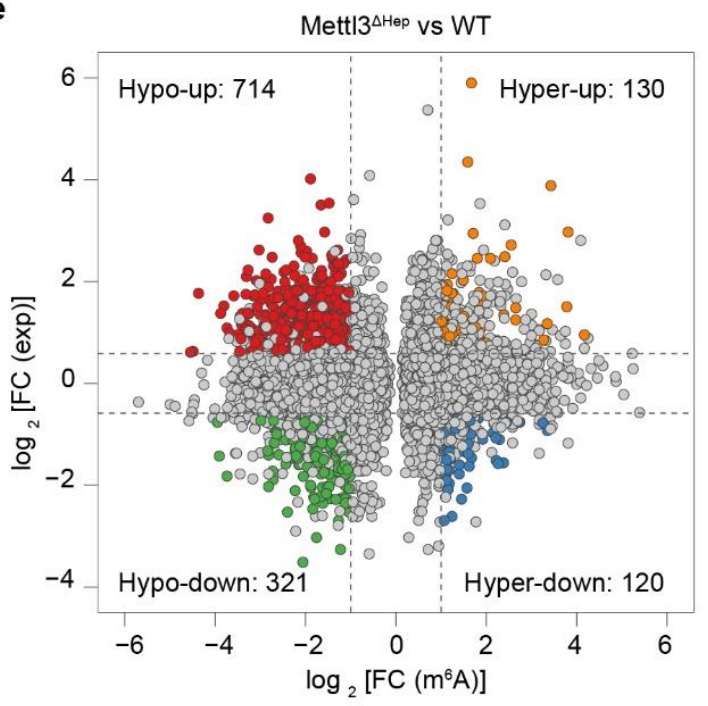

g

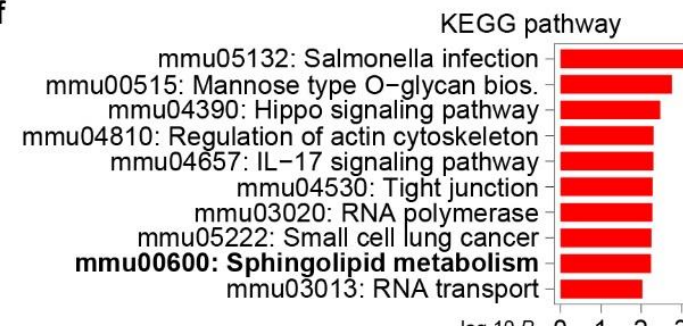

KEGG pathway
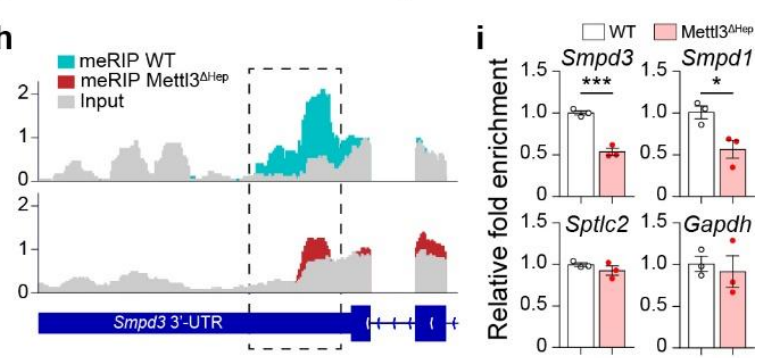

j
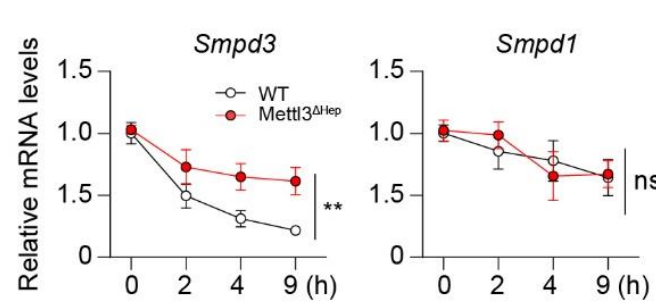

k
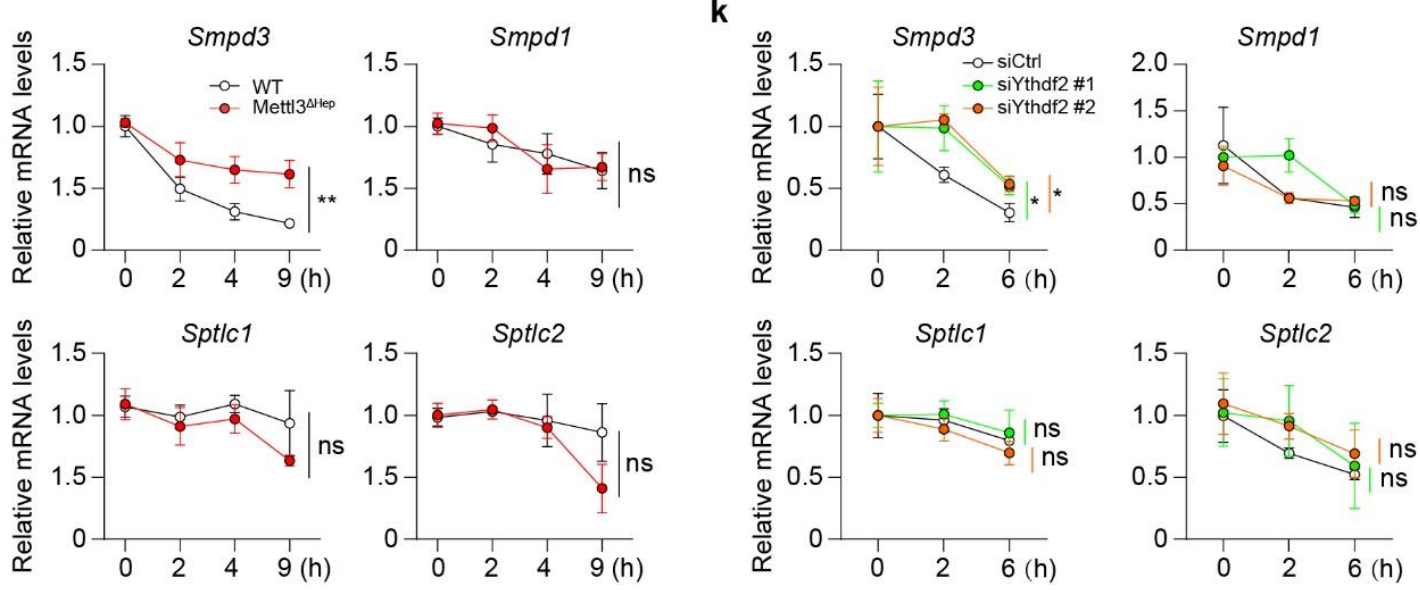

Fig. 4: Mettl3 regulates $S m p d 3$ mRNA decay through $m^{6} A$ modification. a, The enriched consensus motifs were detected within $\mathrm{m}^{6} \mathrm{~A}$ peaks. Statistical analyses were performed using one-tailed binomial test. $\mathbf{b}$, Metagene plot of the frequency of $\mathrm{m}^{6} \mathrm{~A}$ sites throughout the transcript body. c, Pie chart depicting the distribution of $\mathrm{m}^{6} \mathrm{~A}$ peaks in different transcript segments in WT and Mettl3 $3^{\Delta \text { hep }}$ livers. Outer track, WT; inner ring, Mett|3 $3^{\Delta \text { hep }}$. d, Cumulative distribution and boxplot representing the expression changes in transcripts with or without $\mathrm{m}^{6} \mathrm{~A}$ peaks between WT and Mettl3 deficient livers. Statistical 
analysis was performed using two-tailed Kolmogorov-Smirnov test. e, Four quadrant graphs showing the distribution of transcripts with significant changes in both $\mathrm{m}^{6} \mathrm{~A}$ level and expression. All genes with significant differences in gene expression (fold change $>$ 1.5 or $<0.67$ and $p<0.05$ ) and $m^{6} A$ levels (fold change $>2$ or $<0.5$ and $p<0.05$ ) were divided into four groups that included 250 hyper-methylated $\mathrm{m}^{6} \mathrm{~A}$ peaks in mRNA transcripts that were significantly up-regulated (130; hyper-up) or down-regulated (120; hyper-down) and 1035 hypo-methylated $\mathrm{m}^{6} \mathrm{~A}$ peaks in mRNA transcripts that were significantly up-regulated (714; hypo-up) or down-regulated (321; hypo-down). f, Enrichment of KEGG pathways on the 714 genes in the Hypo-up group from (e). g, Bar plot representing the changes in $\mathrm{m}^{6} \mathrm{~A}$ abundance of genes involved in sphingolipid metabolism in WT and Mettl $3^{\text {Shep }}$ livers. Asterisks represent genes that contain $\mathrm{m}^{6} \mathrm{~A}$ peaks with significant changes. $\mathbf{h}, \mathrm{m}^{6} \mathrm{~A}$ abundance on $S m p d 3 \mathrm{mRNA}$ in Mettl3 $3^{\Delta \text { hep }}$ versus WT livers. The y axis represents the normalized $m^{6} A$ signal along the gene. $i, m^{6} A$ enrichment of the indicated mRNAs in Mettl $3^{\Delta \text { hep }}$ versus WT livers by $m^{6} A-R I P-q P C R(n=3)$. Results are presented relative to those obtained with immunoglobulin $G(\lg G)$. Gapdh, $\mathrm{m}^{6} \mathrm{~A}$ negative control. j, mRNA stability analysis in primary hepatocytes isolated from Mettl $3^{\Delta \text { hep }}$ versus WT mice treated with actinomycin $D(5 \mu \mathrm{g} / \mathrm{mL})$ for the indicated times $(n=3)$. The residual RNAs were normalized to the value of time $0 . \mathbf{k}, \mathrm{mRNA}$ degradation assay for the indicated targets in Ythdf2-silenced primary hepatocytes treated with actinomycin $D$ (5 $\mu \mathrm{g} / \mathrm{mL})$ for the indicated times $(n=3)$. The residual RNAs were normalized to the value of time 0. Data are shown in mean \pm SEM; ${ }^{*} p<0.05,{ }^{* *} p<0.01,{ }^{* * *} p<0.001,{ }^{* * *} p<0.0001$ by Kolmogorov-Smirnov test (in the left panel of $d$ ) or Student's t test (in the right panel of $\mathbf{d}, \mathbf{g}$ and $\mathbf{i}$ ) or by two-way ANOVA statistics ( $\mathbf{j}$ and $\mathbf{k}$ ). 
a

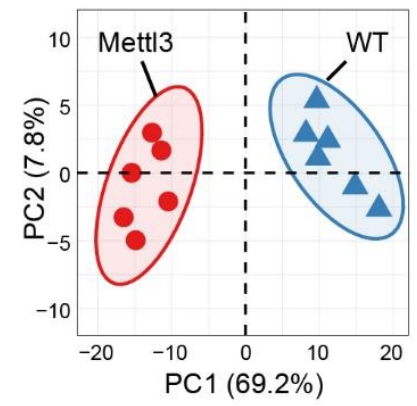

b

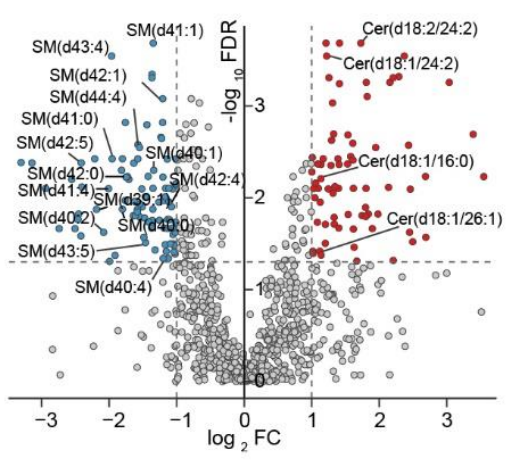

SM

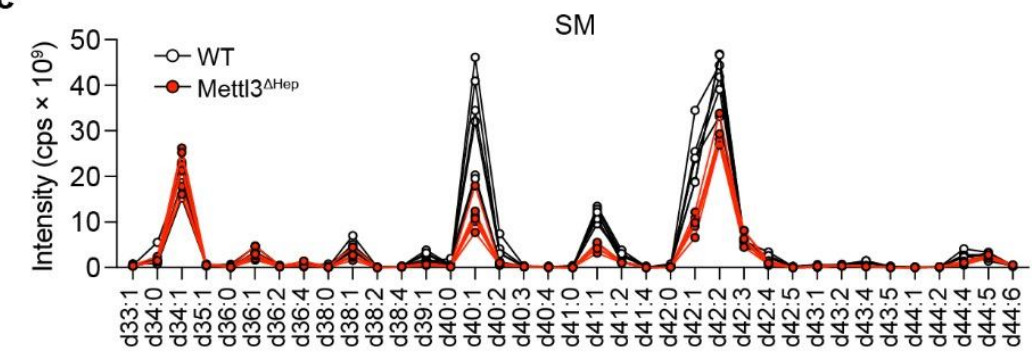

d

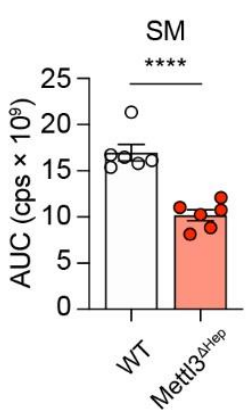

e
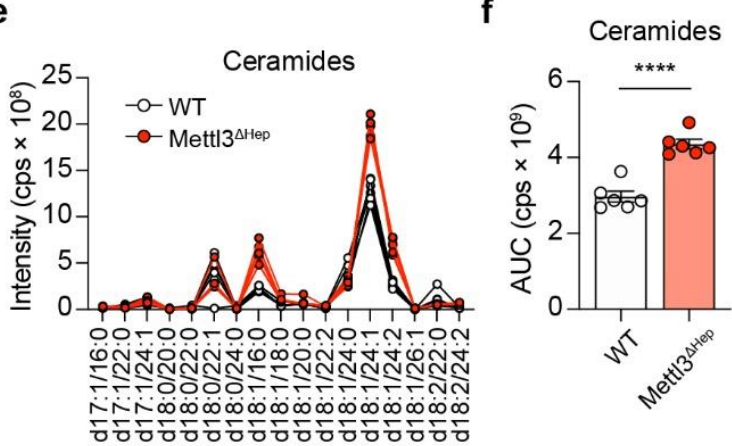

g
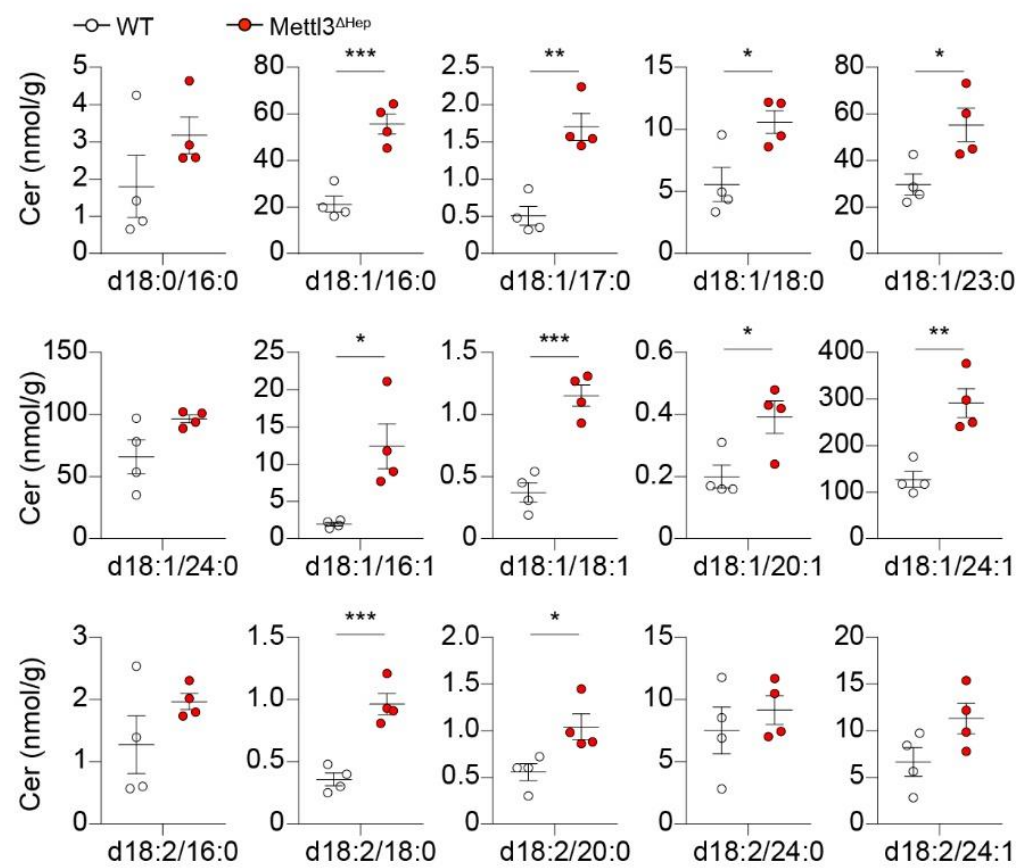

Fig. 5: Hepatic ceramide levels are increased in Mettl|3 ${ }^{\text {hhep }}$ liver. a, Biplot of principal component analysis (PCA) performed on untargeted lipidomic profiling of liver extracts from WT and Mettl $3^{\triangle \text { hep }}$ mice. The red data points highlight Mett $3^{\triangle \text { hep }}$ livers $(n=6)$, wheares 
859 the blue data points highlight WT livers $(n=6)$. $\mathbf{b}$, Volcano plot of untargeted lipidomic 860 profiling of WT and Mettl $3^{\triangle \text { hep }}$ livers. The logarithmic ratios of average fold changes are 861 reported on the $X$ axis. The $y$-axis represents negative logarithmic false-discovery-rate (q) 862 values from the $t$ test performed on six biological replicates. Up- and down-regulated lipid 863 species are highlighted by the red and blue dots, respectively. c, d, lon chromatograms of 864 ceramides (c) and quantification by analysis of AUC (d) in WT and Mettl $3^{\triangle \text { hep }}$ livers. e, $\mathbf{f}$, 865 Ion chromatograms of SM (e) and quantification by analysis of AUC (f) in WT and Mettl $3^{\Delta}$ 866 hep livers. g, Liver content of specific ceramide species from WT and Mettl $3^{\triangle \text { hep }}$ mice. $P$ 867 values by two-sided Student's t-test. * $P<0.05$; ${ }^{* *}, P<0.01$; ${ }^{* \star *}, P<0.001$; ${ }^{* \star * *}, P<$ 8680.0001. 
a

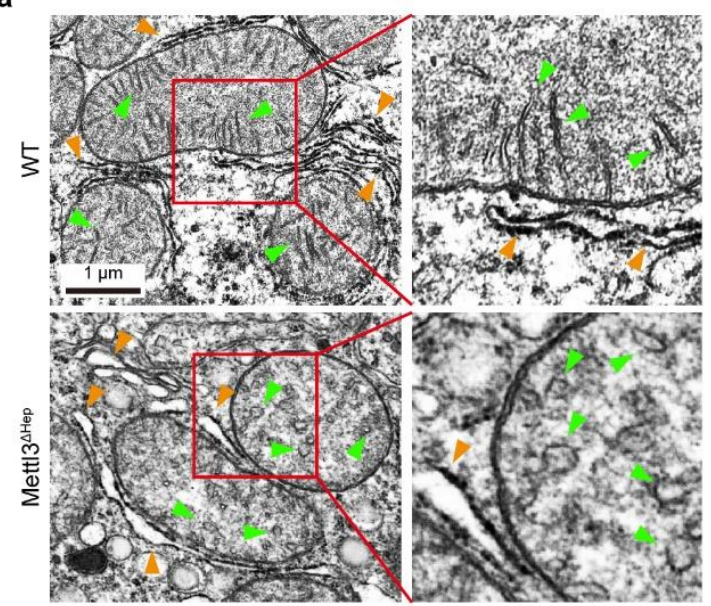

c

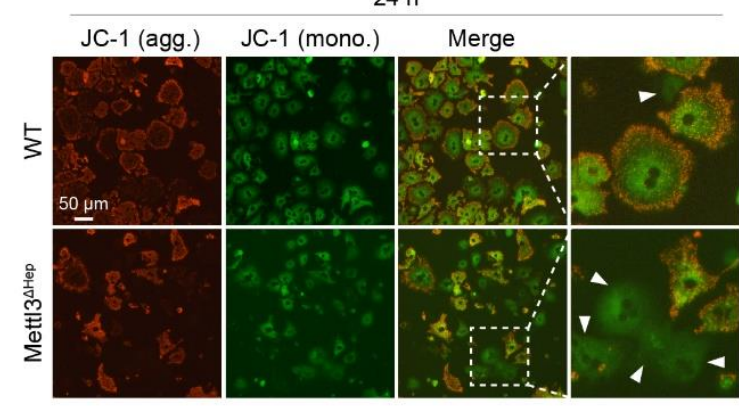

d

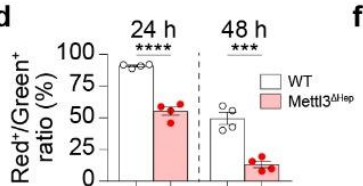

e

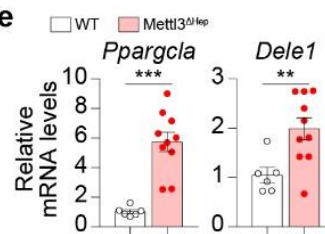

h

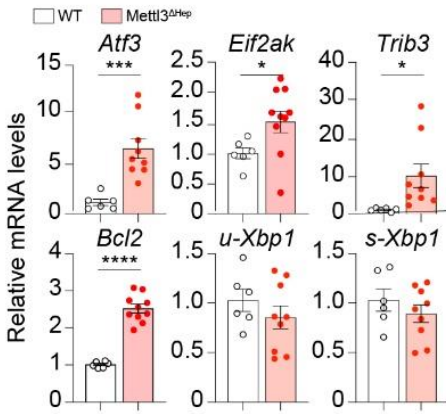

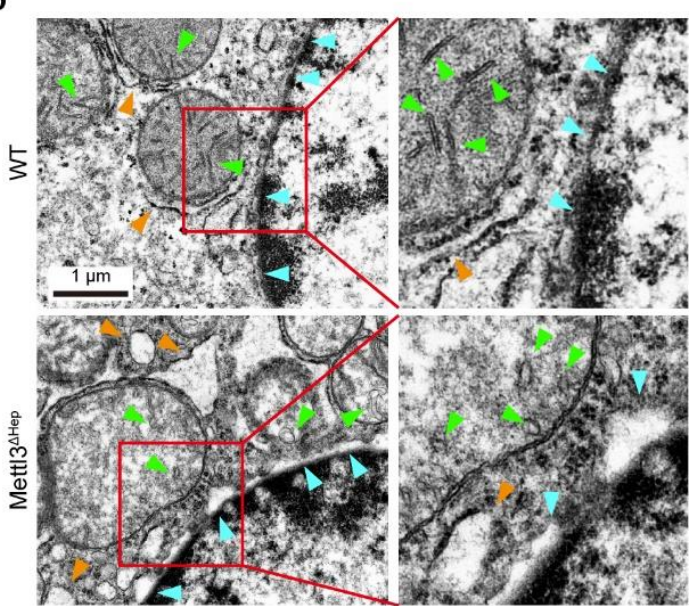

$48 \mathrm{~h}$

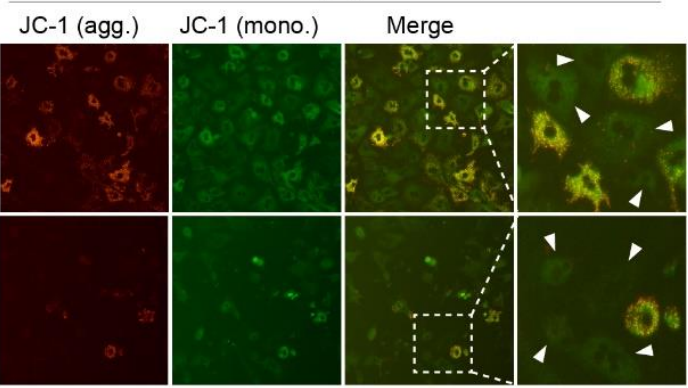

g

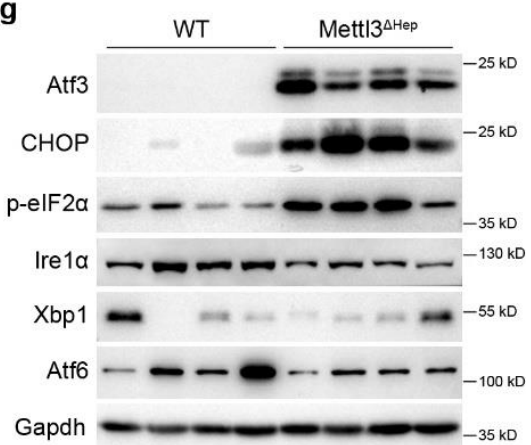

j

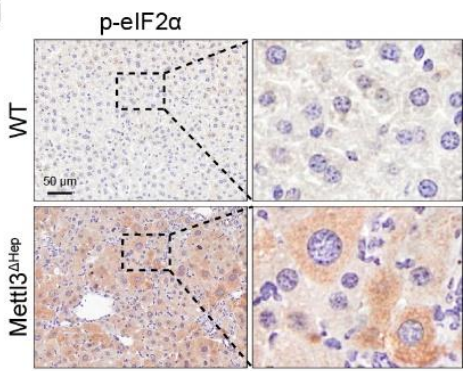

Fig. 6: Mettl3 deficiency in hepatocytes results in mitochondrial damage and ER stress. a, Electron microscopy of WT and Mettl $3^{\text {Ahep }}$ livers. Green arrowheads, mitochondrial cristae; and orange arrowheads, ER. Scale bar, $1 \mu \mathrm{m}$. b. Electron microscopy of WT and Mettl $3^{\text {hep }}$ livers. Green arrowheads, mitochondrial cristae; orange arrowheads, ER; and blue arrowheads, perinuclear space. Scale bar, $1 \mu \mathrm{m}$. c, Mitochondrial membrane potential assessment of primary hepatocytes from WT and Mettl3 shep mice at 24 and 48 hours after isolation with the mitochondria-specific probe JC-1. Red 
877 and green fluorescence indicate J-aggregates and JC-1 monomers, respectively. Scale bar,

$87850 \mu \mathrm{m}$. d, The red to green fluorescence intensity ratio from (c) was used to indicate the 879 mitochondrial membrane potential. e, qRT-PCR of livers from WT and Mettl $3^{\text {Ahep }}$ mice for 880 indicated genes. $\mathbf{f}$, IHC of MDA in WT and Mettl $3^{\Delta \text { hep }}$ livers. Scale bar, $50 \mu \mathrm{m}$. $\mathbf{g}$, Western 881 blot of liver lysates from WT and Mettl ${ }^{\triangle \text { hep }}$ mice for indicated proteins. $\mathbf{h}$, qRT-PCR of livers

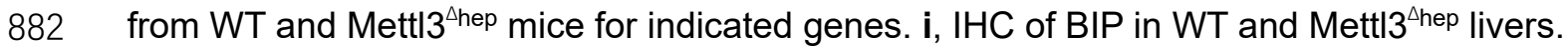
883 Scale bar, $50 \mu \mathrm{m}$. j, IHC of p-eIF2 $\alpha$ in WT and Mettl $3^{\triangle \text { hep }}$ livers. Scale bar, $50 \mu \mathrm{m}$. Data are 884 shown in mean \pm SEM; ${ }^{*} p<0.05,{ }^{* *} p<0.01,{ }^{* * *} p<0.001,{ }^{* * * *} p<0.0001$ by Student's $t$ 885 test. 
a
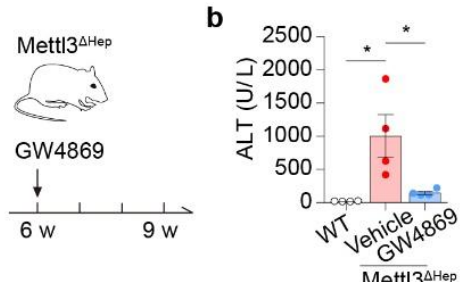

d Vehicle e GW4869 Mettl3 ${ }^{\text {Hep }}$

d

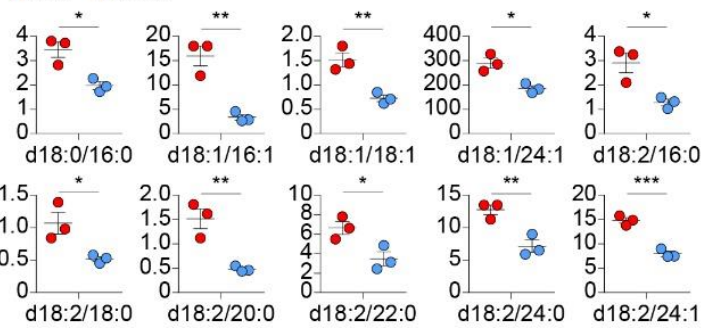

e

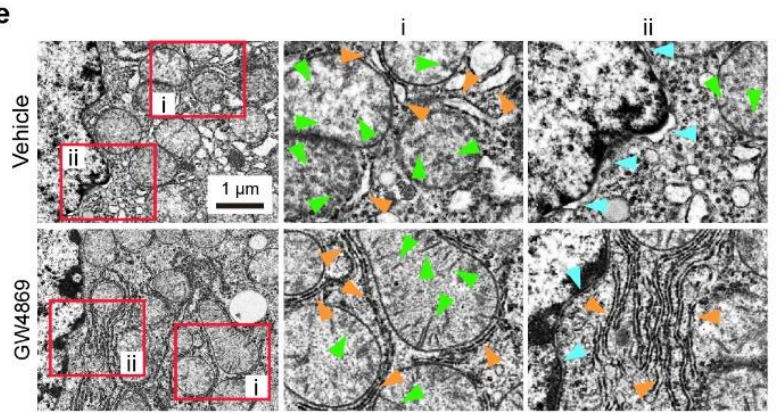

g

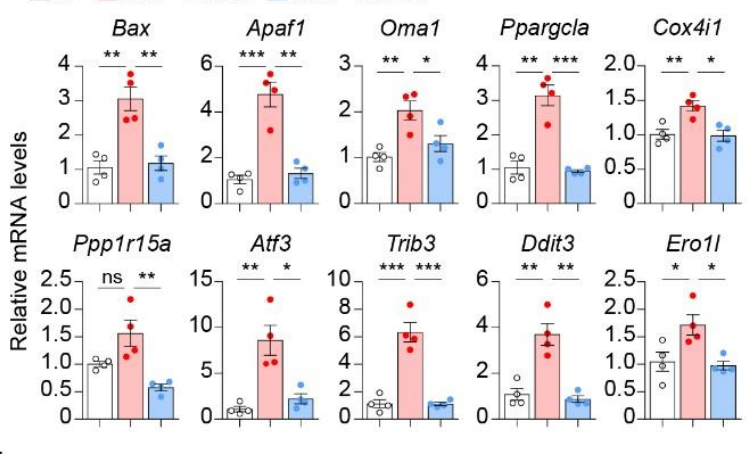

i

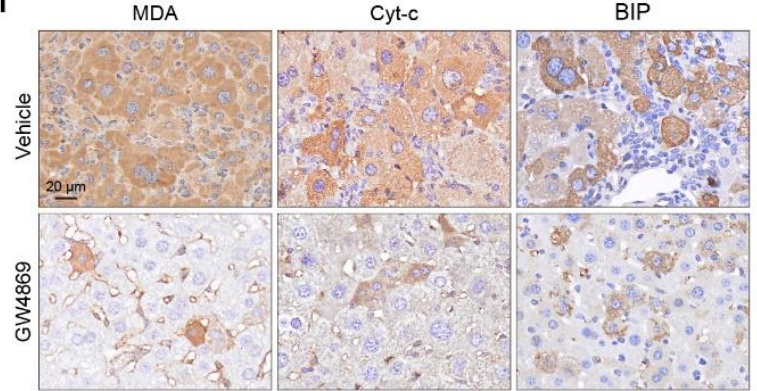

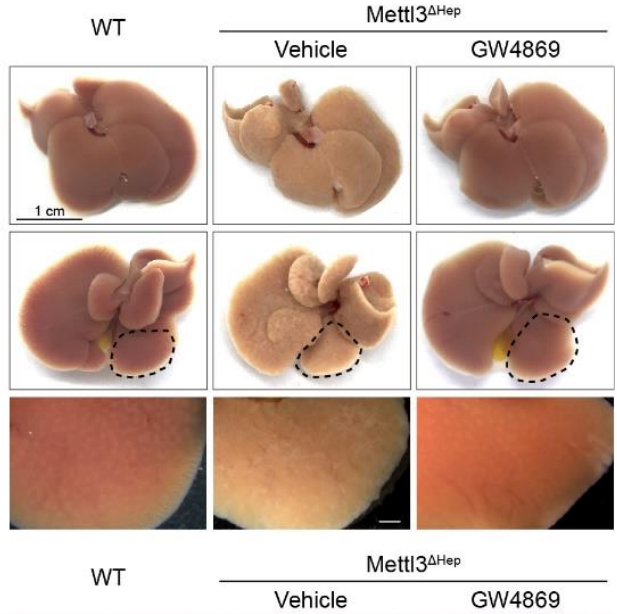

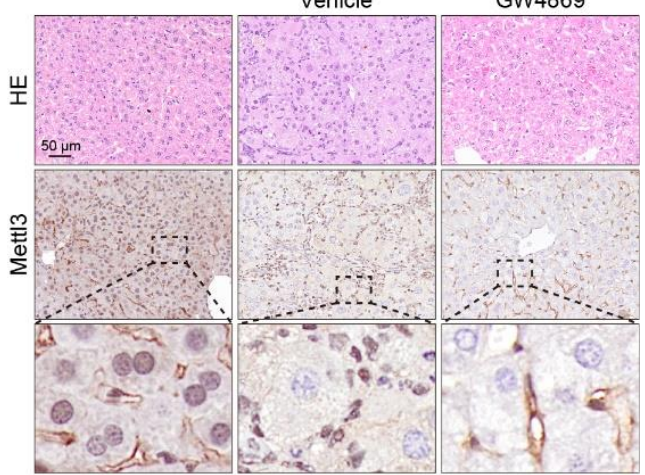

h
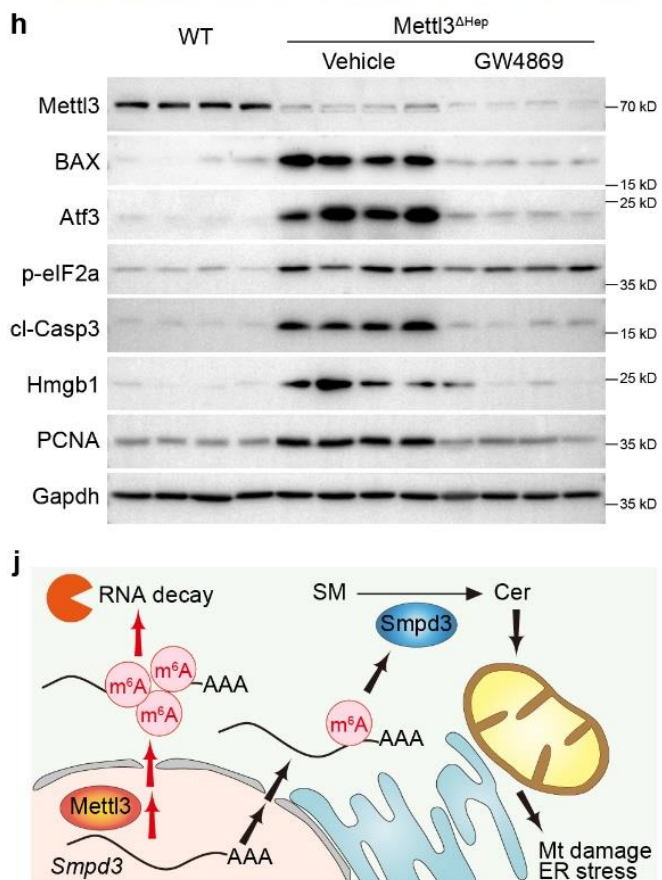

Fig. 7: Pharmacological inhibition of Smpd3 attenuates Mettl3 deficiency-induced ceramide accumulation and liver injury. a, Timeline of GW4869 treatment on Mettl $3^{\triangle \text { hep }}$ mice. b, Liver injury was assessed by serum AST and ALT in Mettl $3^{\Delta \text { hep }}$ mice injected with GW4869 for 3 weeks and sacrificed $24 \mathrm{~h}$ after final injection. c, Representative liver macroscopy. Scale bar, $1 \mathrm{~cm}$. d, Liver content of specific ceramide species from Mettl $3^{\Delta \text { hep }}$ mice treated with GW4869 versus vehicle. e, Electron microscopy of Mettl $3^{\text {thep }}$ livers treated with GW4869 versus vehicle. Green arrowheads, mitochondrial cristae; organce 
894 arrowheads, ER; and blue arrowheads, perinuclear space. Scale bar, $1 \mu \mathrm{m}$. f, H\&E and 895 Mettl3 IHC in livers from mice in (b). j, qRT-PCR of livers from mice in (b for indicated 896 genes. $\mathbf{h}$, Western blot of liver lysates from mice in (b) for indicated proteins. i, MDA, Cyt$897 \mathrm{C}$ and BIP IHC in livers from Mettl $3^{\Delta \text { hep }}$ mice treated with GW4869 or vehicle. j, Model 898 depicting the role of Mett $3 / \mathrm{m}^{6} \mathrm{~A} / \mathrm{Smpd} 3$ axis in regulating sphingolipid metabolic 899 homeostasis during postnatal liver development. Data are shown in mean \pm SEM; ns, not 900 significant, ${ }^{*} p<0.05,{ }^{* *} p<0.01,{ }^{* * *} p<0.001$ by Student's $t$ test.

901 
902

903

904

905

906

907

908

909

910

\section{Supplemental Information}

$\mathrm{m}^{6} \mathrm{~A}$ modification-tuned sphingolipid metabolism regulates postnatal liver development

Shiguan Wang, Shanze Chen, Jianfeng Sun, Pan Han, Bowen Xu, Youquan Zhong, Zaichao Xu, Peng Zhang, Ping Mi, Cuijuan Zhang, Yuchen Xia, Shiyang Li, Mathias Heikenwälder, Detian Yuan 
a

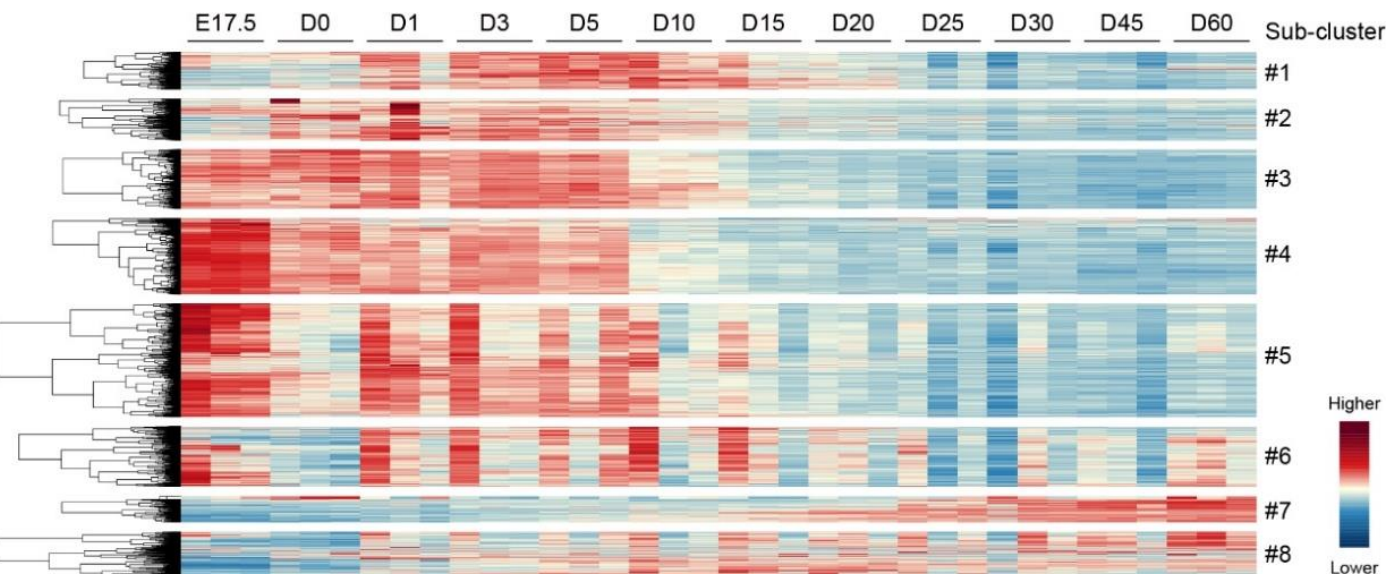

b

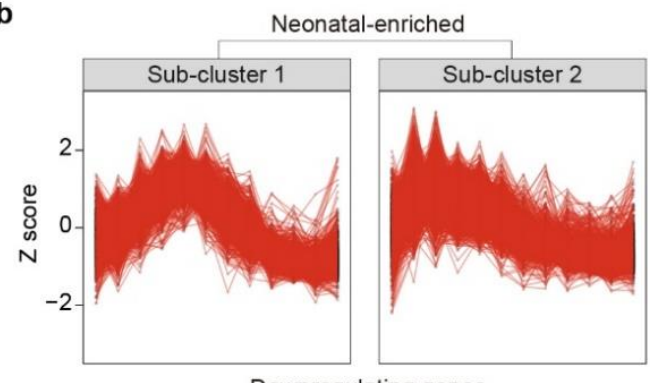

Downregulating genes
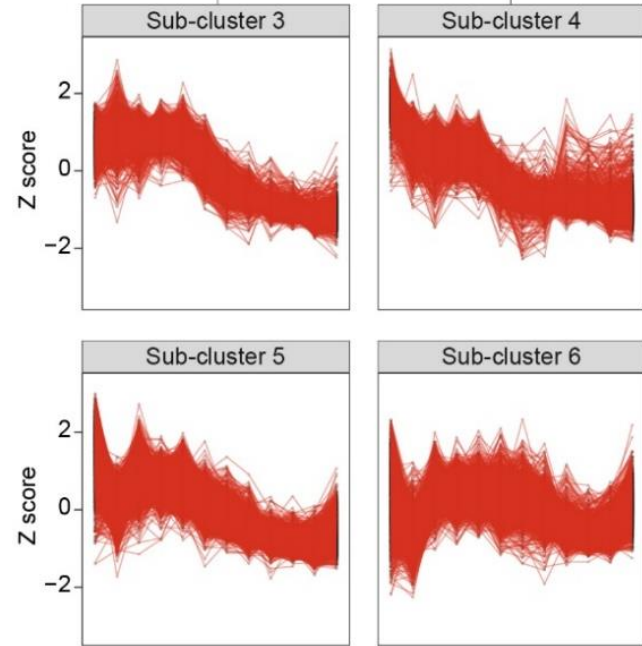

Upregulating genes
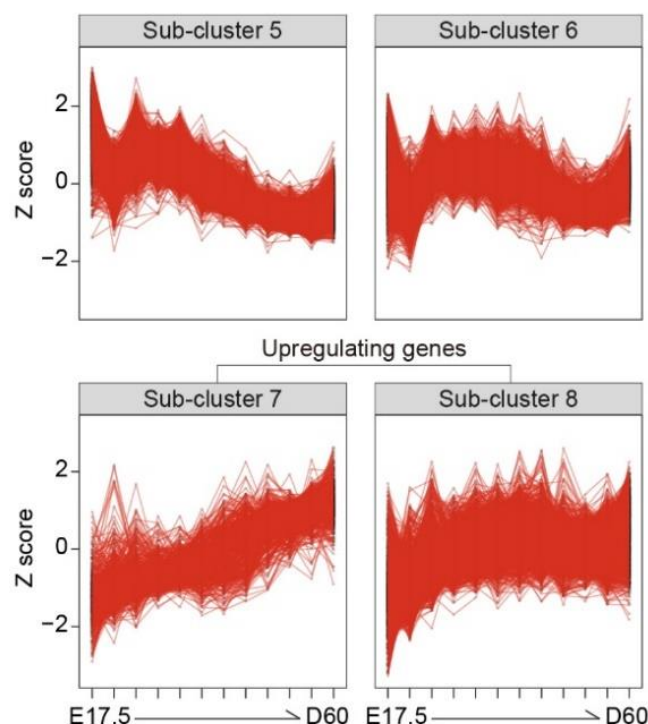

C

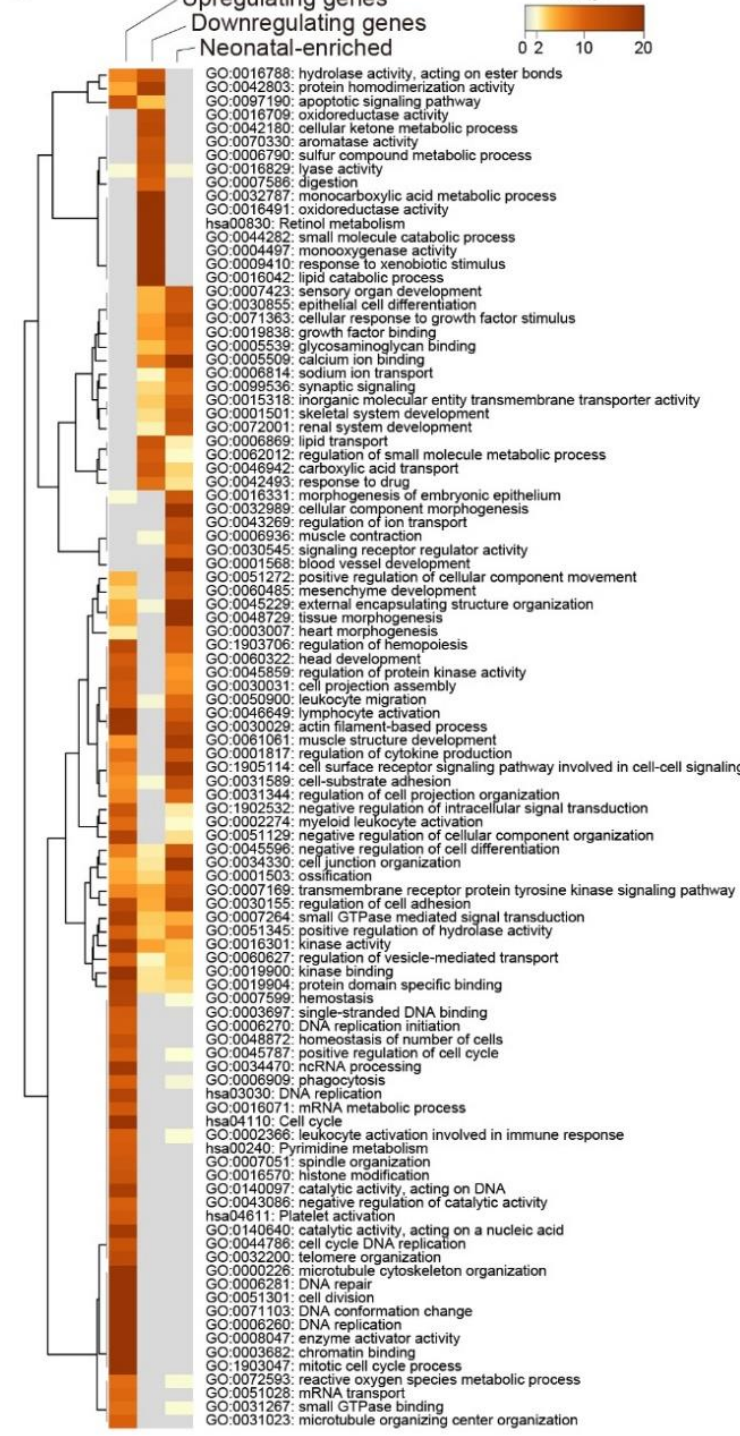

Extended Data Fig. 1: Gene expression patterns in mouse liver development

a, Eight $k$-means clusters showing different expression trends in murine livers at different stages of development determined by the normalized gene expression using the Z-score transformation method. $\mathbf{b}$, The average temporal expression patterns of genes in the eight clusters. c, Heatmap of enriched GO and KEGG terms colored by $p$-values. 
a
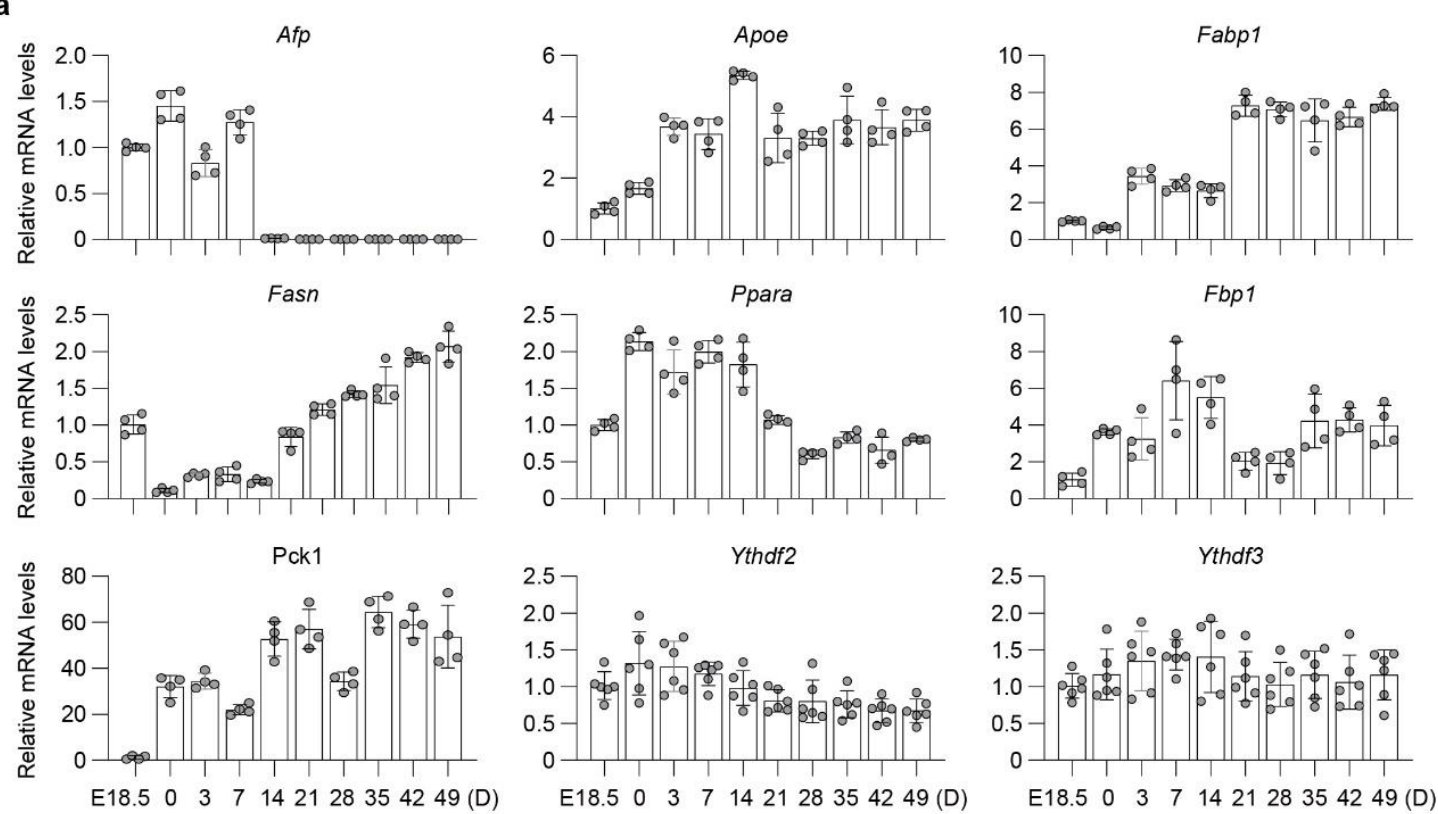

b

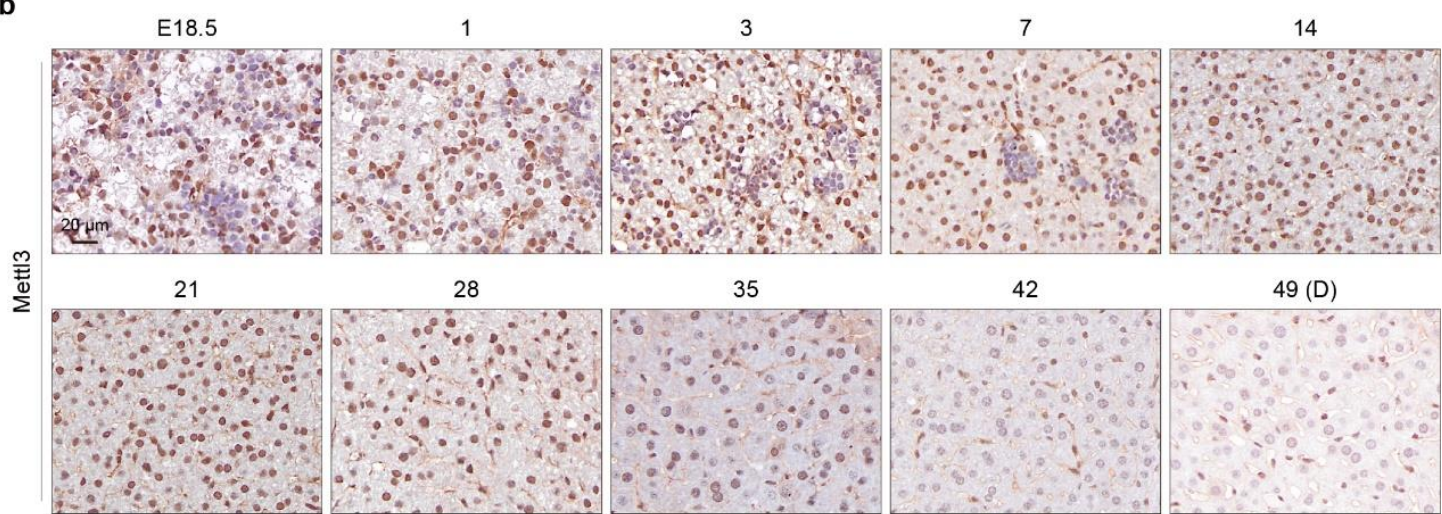

c

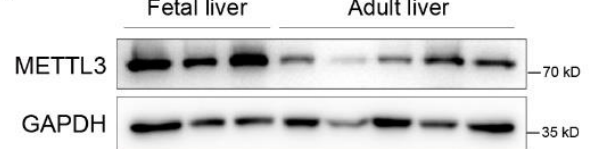

919 Extended Data Fig. 2: Dynamic changes of Mettl3 expression during postnatal liver development

921 a, qRT-PCR of livers from WT C57BL/6J mice at different ages for indicated genes. b, IHC of Mettl3 in livers from WT C57BL/6J mice at different ages as indicated. Scale bar, $20 \mu \mathrm{m}$. c, Immunoblotting of METTL3 in human fatal and adult liver samples. GAPDH was used as a loading control. 
a
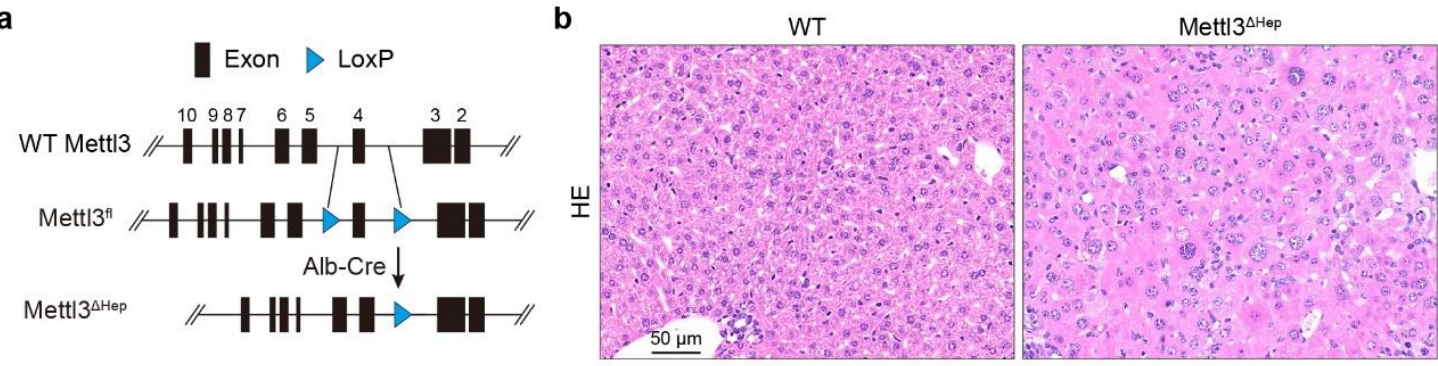

c

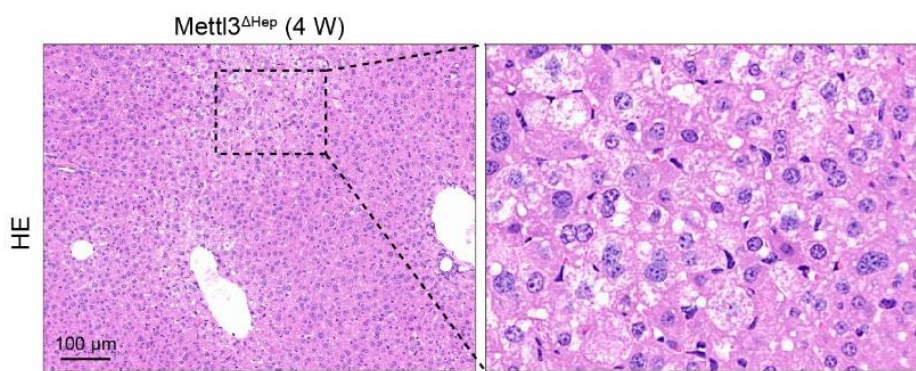

e

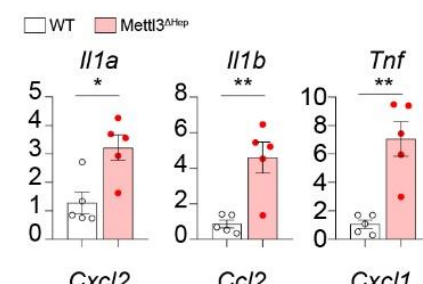

d

CD3

B220

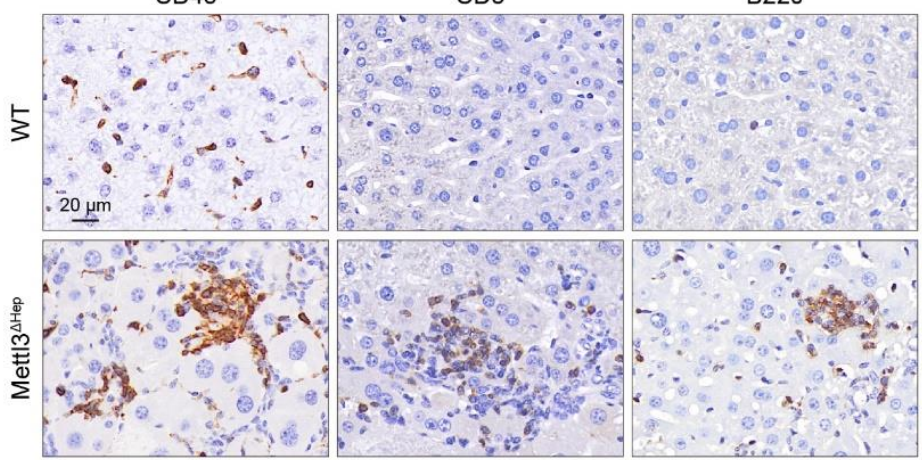

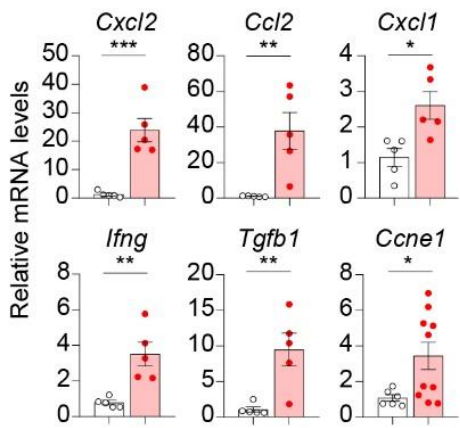
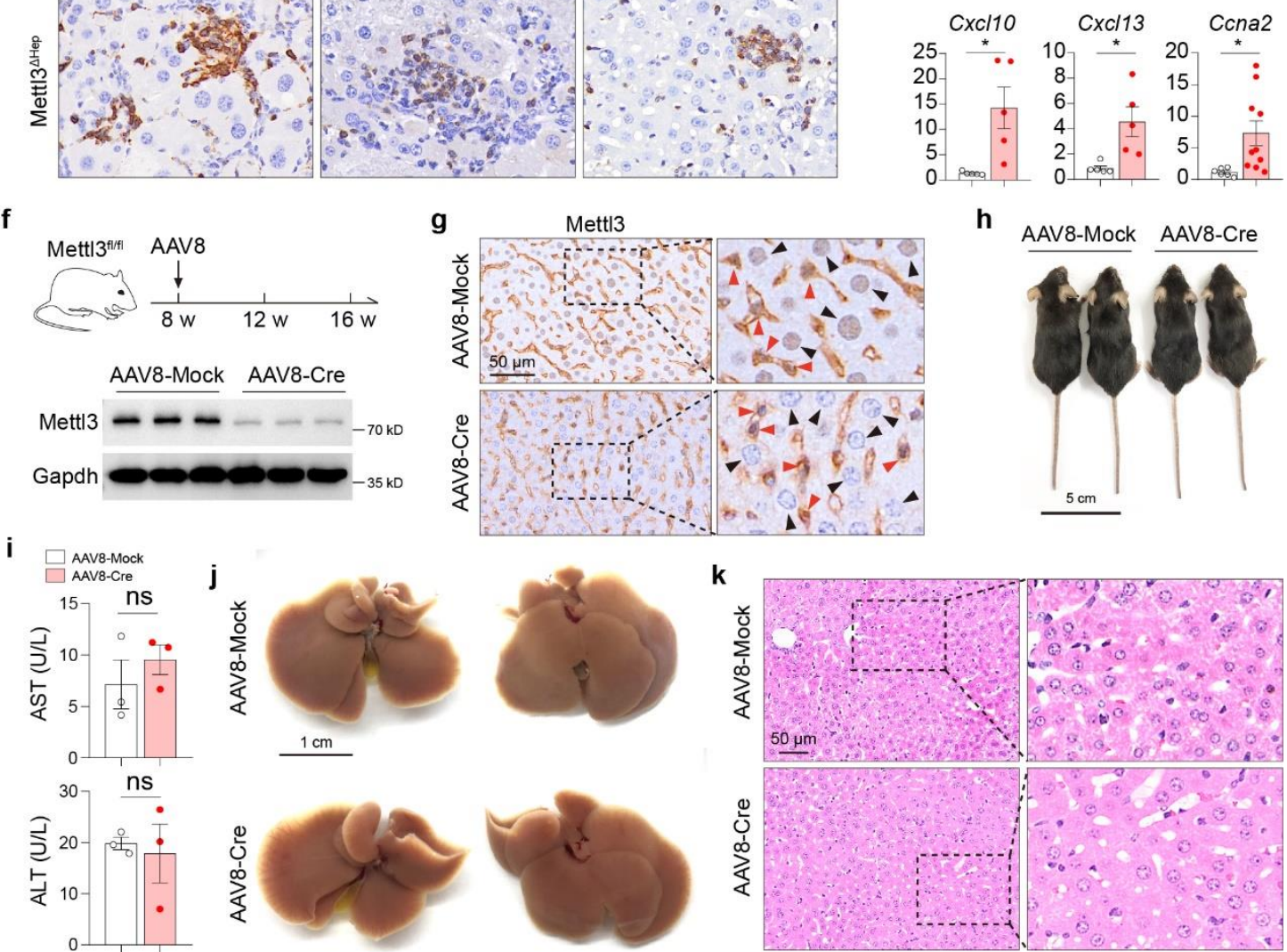

Extended Data Fig. 3: Hepatic Mettl3 deficiency induces hepatocyte hypertrophy 
929 (bottom) alleles. $\mathbf{b}, \mathrm{H} \& \mathrm{E}$ staining of liver sections from 5-week-old WT and Mettl $3^{\text {Ahep }}$ mice.

930 Scale bar, $50 \mu \mathrm{m}$. c, H\&E staining of liver sections from 4-week-old WT and Mettl $3^{\triangle \text { hep }}$ mice.

931 Scale bar, $100 \mu \mathrm{m}$. d, IHC of CD45, CD3 and B220 in livers from WT and Mettl $3^{\text {Lhep }}$ mice.

932 Scale bar, $20 \mu \mathrm{m}$. e, qRT-PCR of livers from WT and Mettl $3^{\text {thep }}$ mice for indicated genes.

933 f, Timeline of AAV8-induced Cre expression in Mett/3/f/fl mice and immunoblotting for Mettl3

934 showing the Knock-out effect. g, IHC of Mettl3 in livers from AAV8-Mock and AAV8-Cre

935 Mett// $3^{\text {fl/fl }}$ mice. Black arrowheads, hepatocytes; Red arrowheads, non-parenchymal cells.

936 Scale bar, $50 \mu \mathrm{m}$. h, Representative picture of AAV8-Mock and AAV8-Cre Mett//fl/fl mice 2

937 months after i.v. injection. Scale bar, $5 \mathrm{~cm}$. i, Serum ALT and AST in AAV8-Mock and AAV8-

938 Cre Mett/ $3^{f / / f l}$ mice. j, Gross appearance of livers in AAV8-Mock and AAV8-Cre Mett/3///fl mice

9392 months after i.v. injection. The scale bar represents $1 \mathrm{~cm}$. $\mathbf{k}$, Representative H\&E staining

940 of livers from AAV8-Mock and AAV8-Cre Mett/3/f/ll mice. Scale bar, $50 \mu \mathrm{m}$. Data are shown

941 in mean \pm SEM; ns, not significant, ${ }^{*} p<0.05,{ }^{* *} p<0.01,{ }^{* * *} p<0.001$ by Student's t test. 
a

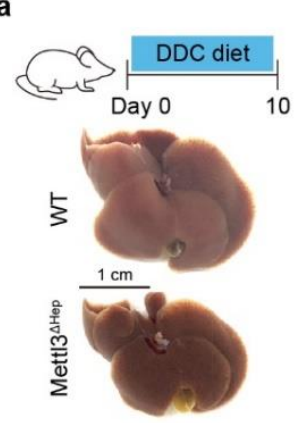

d

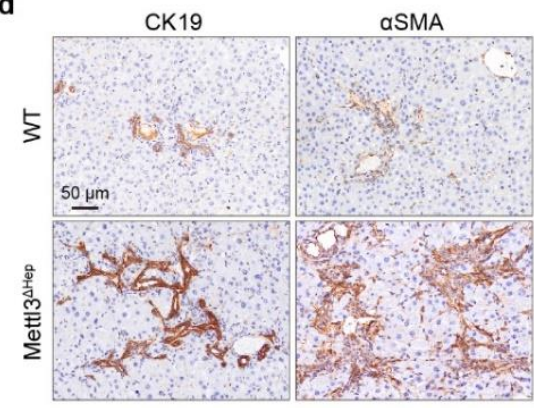

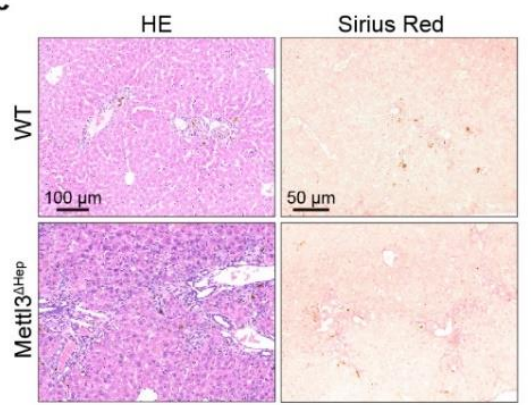

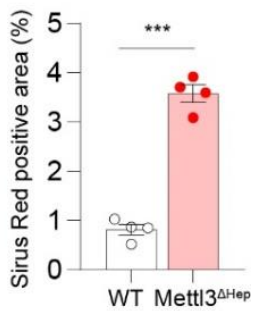

e

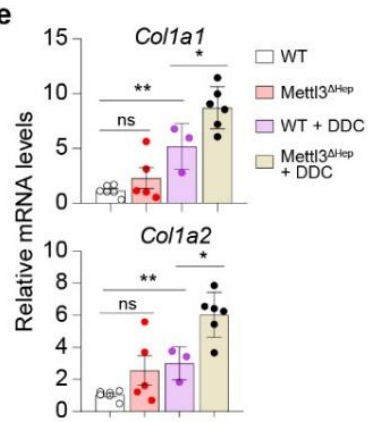

f

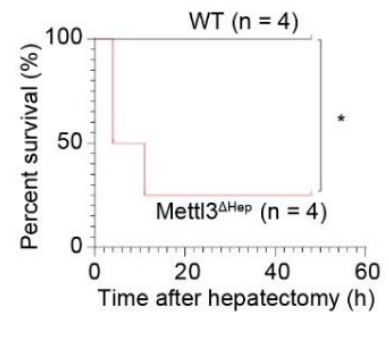

g

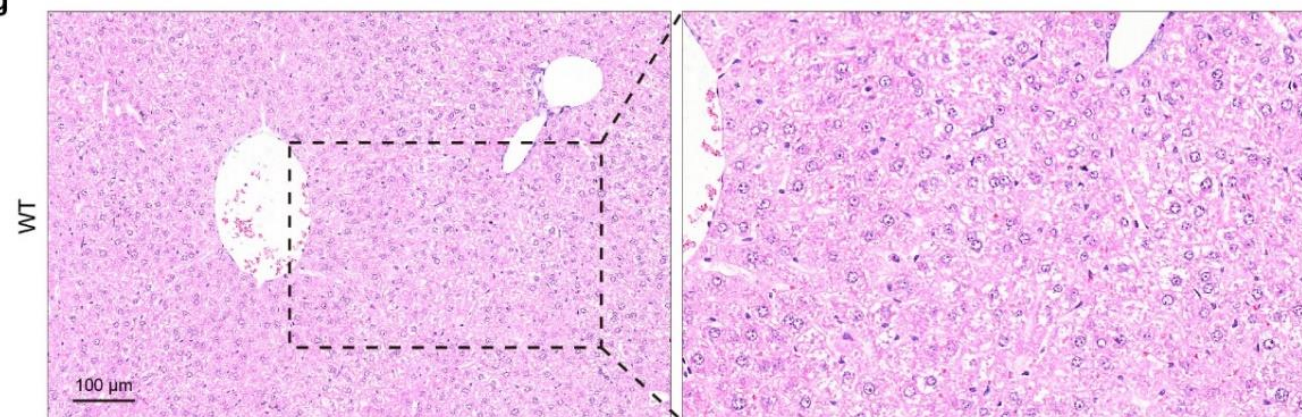

\section{Extended Data Fig. 4: Hepatic Mettl3 deficiency induces liver injury}

a, Representative macroscopy of livers from WT and Mettl $3^{\Delta \text { hep }}$ mice on DDC diet. $\mathbf{b}$, Serum ALT and AP in WT and Mett $3^{\triangle \text { hep }}$ mice on DDC diet. c, H\&E and Sirius Red staining of livers from WT and Mettl $3^{\text {thep }}$ mice on DDC diet. Sirius Red staining was quantified using Image J. Scale bar, $100 \mu \mathrm{m}$ (left), $50 \mu \mathrm{m}$ (right). d, IHC of CK19 and aSMA in livers from WT and Mettl $3^{\text {Ahep }}$ mice on DDC diet. Scale bar, $50 \mu \mathrm{m}$. e, qRT-PCR of livers from WT and Mett| $3^{\triangle \text { hep }}$ mice on Ctrl versus DDC diet for indicated genes. $\mathbf{f}$, Survival curve of WT and Mett $3^{\triangle \text { hep }}$ mice after partial hepatectomy. g, Representative H\&E staining of livers from WT and Mettl $3^{\triangle \text { hep }}$ mice 5 hours after partial hepatectomy. Scale bar, $100 \mu \mathrm{m}$. Data are shown in mean \pm SEM; ns, not significant, ${ }^{*} p<0.05,{ }^{* *} p<0.01,{ }^{* * *} p<0.001,{ }^{* * *} p<0.0001$ by log rank test (f) or Student's t test (b, $\mathbf{c}$ and $\mathbf{e})$. 

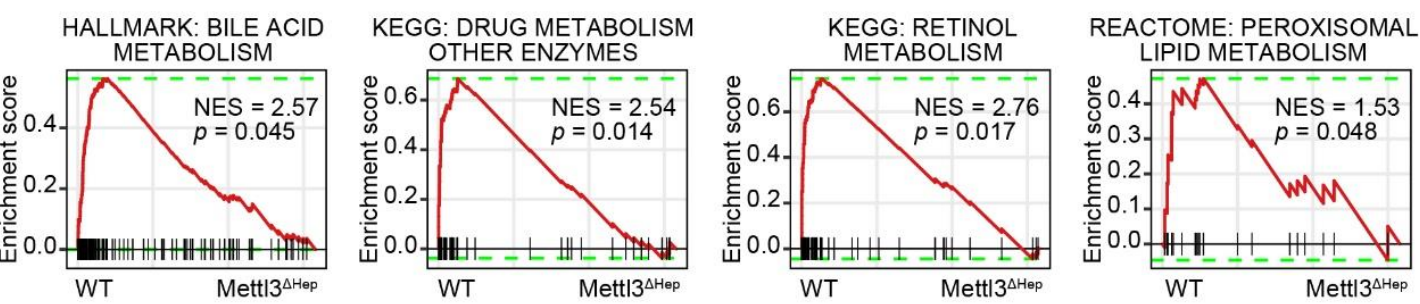

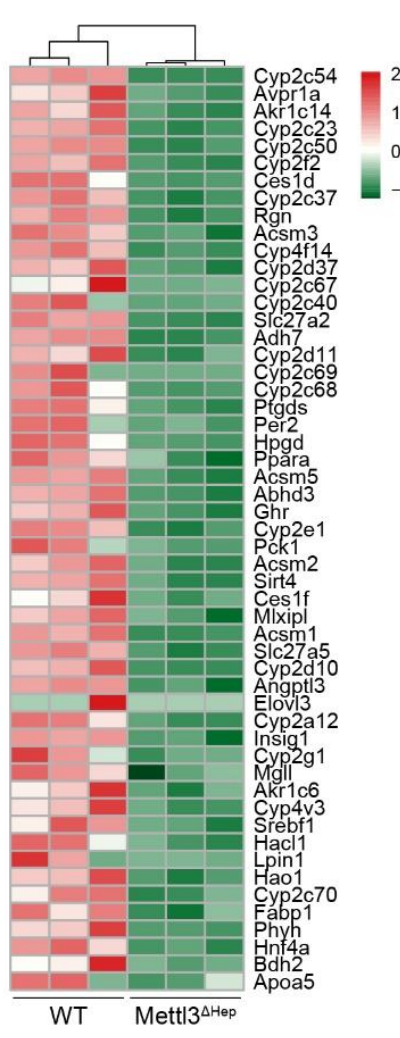

C

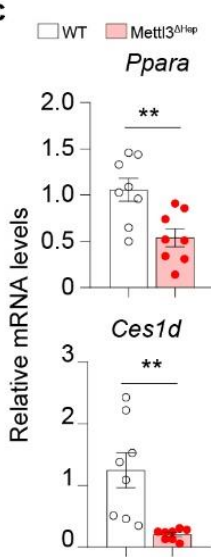

d
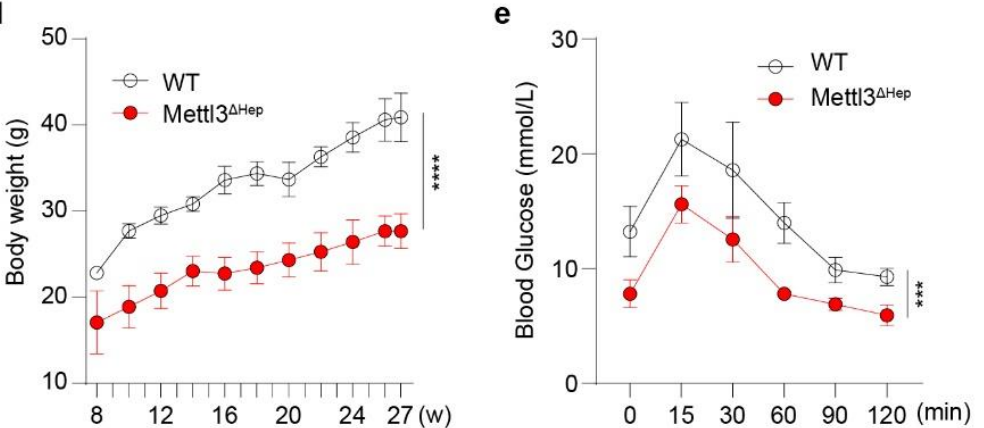

h
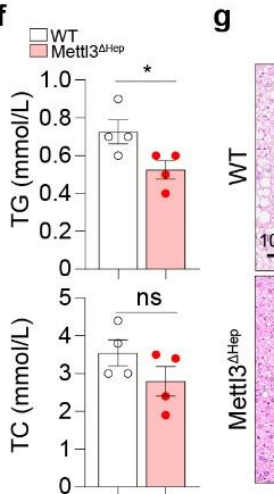

HE
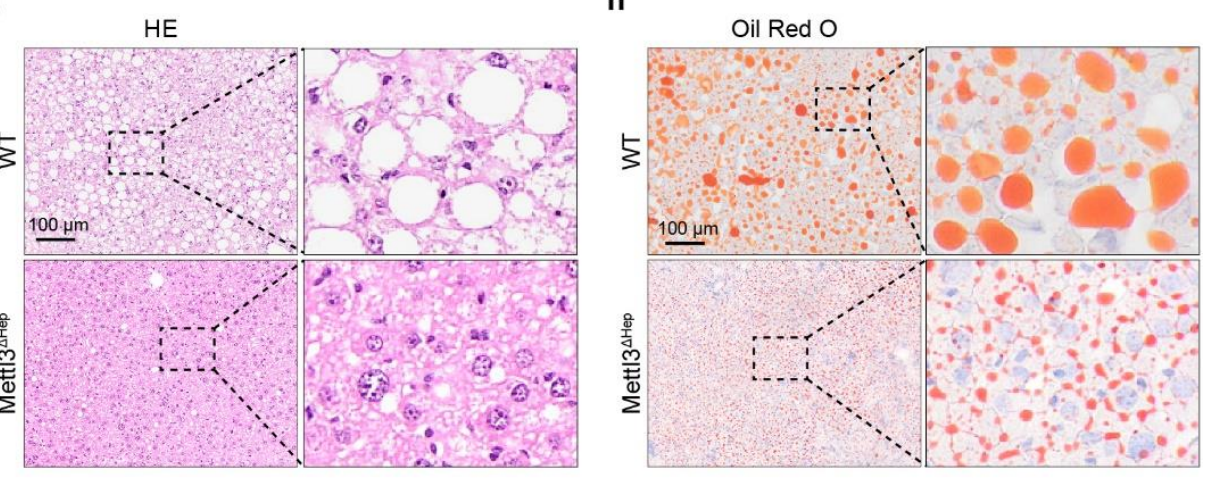

Extended Data Fig. 5: Mettl3 deficiency in hepatocytes leads to metabolic reprogramming

a, GSEA analysis in Mett| $3^{\text {hep }}$ versus WT livers for the indicated gene sets. b, Heat map depicting expression of metabolic genes in WT and Mettl3 $3^{\triangle \text { hep }}$ livers. c, qRT-PCR of WT versus Mett $3^{\triangle \text { hep }}$ livers for indicated genes. d, Weight development in WT and Mett $3^{\triangle \text { hep }}$ mice on HFD. e, Glucose tolerance test in WT and Mettl $3^{\text {hep }}$ mice on HFD for 4 months. $f$, Quantification of serum triglyceride and cholesterol in WT and Mettl $3^{\Delta \text { hep }}$ mice on HFD for 4 months. g, Representative H\&E staining of livers from WT and Mettl $3^{\triangle \text { hep }}$ mice on HFD 
963 for 4 months. Scale bar, $100 \mu \mathrm{m}$. h, Representative Sudan red staining illustrating fat 964 accumulation in livers of WT and Mettl $3^{\triangle \text { hep }}$ mice on HFD for 4 months. Scale bar, $100 \mu \mathrm{m}$.

965 Data are shown in mean \pm SEM; ns, not significant, ${ }^{*} p<0.05,{ }^{* *} p<0.01,{ }^{* * *} p<0.001$, $966{ }^{* * * *} p<0.0001$ by two-way ANOVA statistics ( $\mathbf{d}$ and $\mathbf{e}$ ) or Student's t test (c and $\mathbf{f}$ ). 
Sphingolipid_metabolism obiotics_by_cytochrome_p450 Arachidonic_acid_metabolism Amino_sugar_and_nucleotide_sugar_metabolism
Other_glycan_degradation
N_glycan_biosynthesis Amino_sugar_and_nucleotide_sugar_metabolism
Other_glycan_degradation
N_glycan_biosynthesis Biosynthesis_of_unsaturated_fatty_acids Glycosphingolipid_biosynthesis_globo_series Glycosphingolipid_biosynthesis_lacto_and_neōlacto_series Glycosaminoglycan_biosynthesis__keratan_sulfate Âminoacyl_trna biosynthesis Beta alanine metabolism Glyoxylate_and_dicarboxylate_metabolism Glycosaminoglycan_degradation Gycosaminoglycan_biosynthesis_chondroitin_sulfate Pyrimidine_metabolism One_carbon_pool_by_folate Butanoate_metabolism Purine metabolism Fructose_and_mannose_metabolism Fructose_and mannose_metabolism Pentose phosphate pathway Nicotinate_and nicotinamide metabolism Histidine metabolism Valine leucine and isoleucine-degradation Sēenoāmino acid metabolism Lysine degradation Citrate cycle tca cycle O_glycan biosynthesis O_glycan_biosynthesis olate_biosynthesis Gycosaminoglycan biosynthesis heparan sulfate Glycosylphosphatidylinositol_gpi_anchor_biosynthesis Sulfur_metabolism Rna_polymerase Ether_lipid_metabolism Alpha_linolenic_acid_metabolism Gycerophospholipid_metabolism Galactose metabolism
Phosphatidylinositol signaling system Taurine and hypotaurine metabolism Valine leucine and Gycerolipid metabolism Arginine and proline metabolism Cysteine and methionine metabolism Glycosphingolipid_biosynthesis_ganglio_series Alanine aspartate and Tryptophan_metabolism Alanine_aspartate_and_glutamate_metabolism Gycolysis_gluconeogenesis . Tyrosine_metabolism Glycine serine and threonine metabolism Pantothenate_and_coa_biosynthesis Nitrogen_metabolism Retinol metabolism Steroid_biosynthesis
Terpenoid_backbone_biosynthesis
Primary bile acid_biosynthesis Primary_bile_acid_biosynthesis Ascorbate_and_addarate_metabolism Steroid_hormone_biosynthesis Pentose_and_glucuronate_interconversions
Porphyrin and chlorophyll metabolism Porphyrin_and_chlorophyll_metabolism
Starch_and_sucrose_metabolism

$$
-2.5
$$

Extended Data Fig. 6: Metabolic alterations revealed by transcriptomic profiles of WT and Mett| $3^{\text {Ahep }}$ livers

971 Enrichment of KEGG metabolic signatures in Mett| $3^{\Delta \text { hep }}$ versus WT livers. Signatures that

972 are significantly upregulated (red) or downregulated (blue) are highlighted. 
a

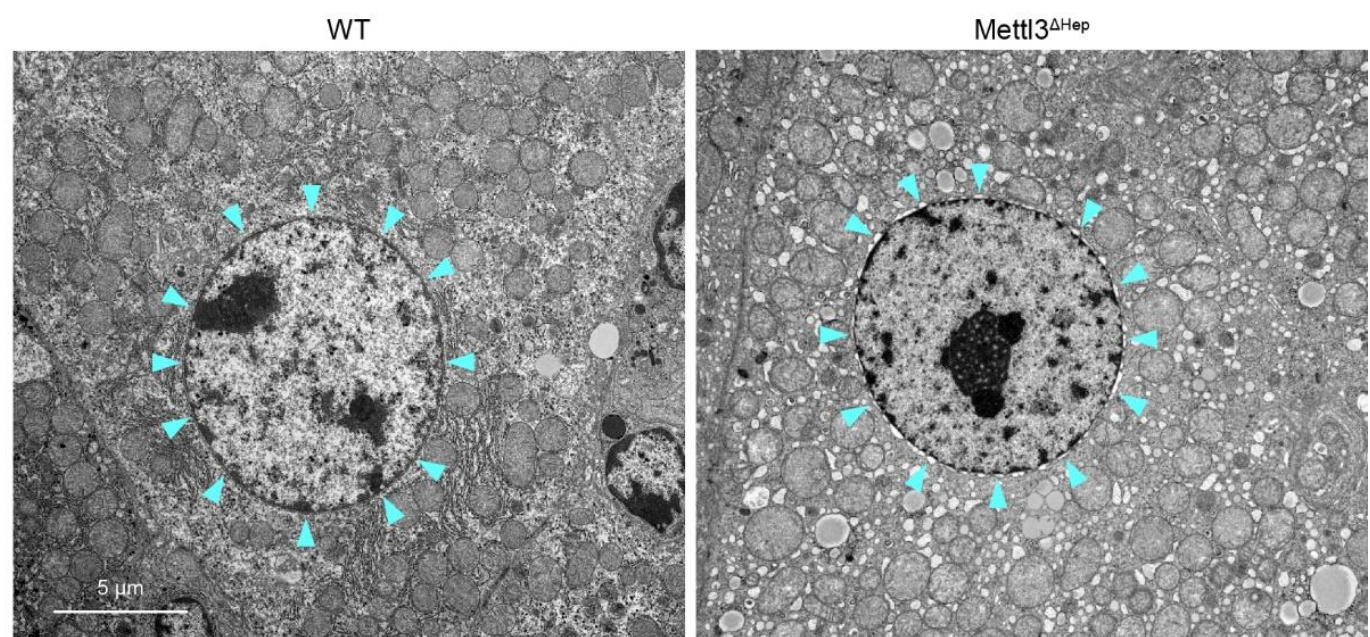

b
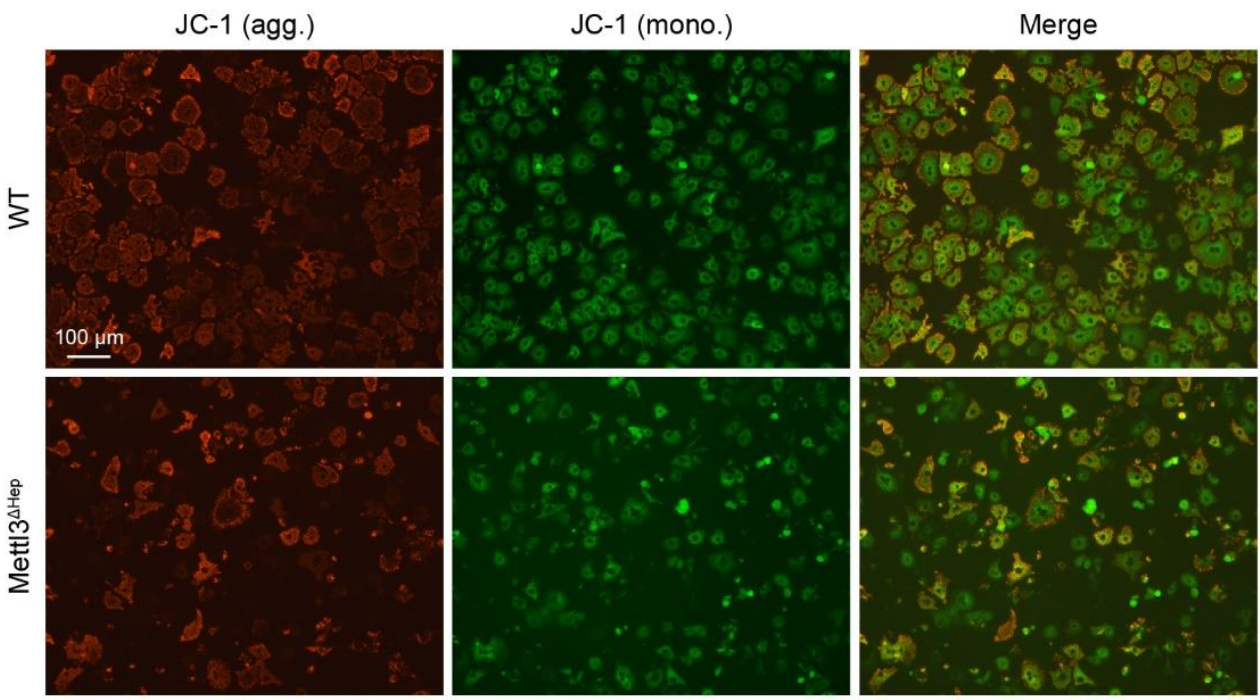

C
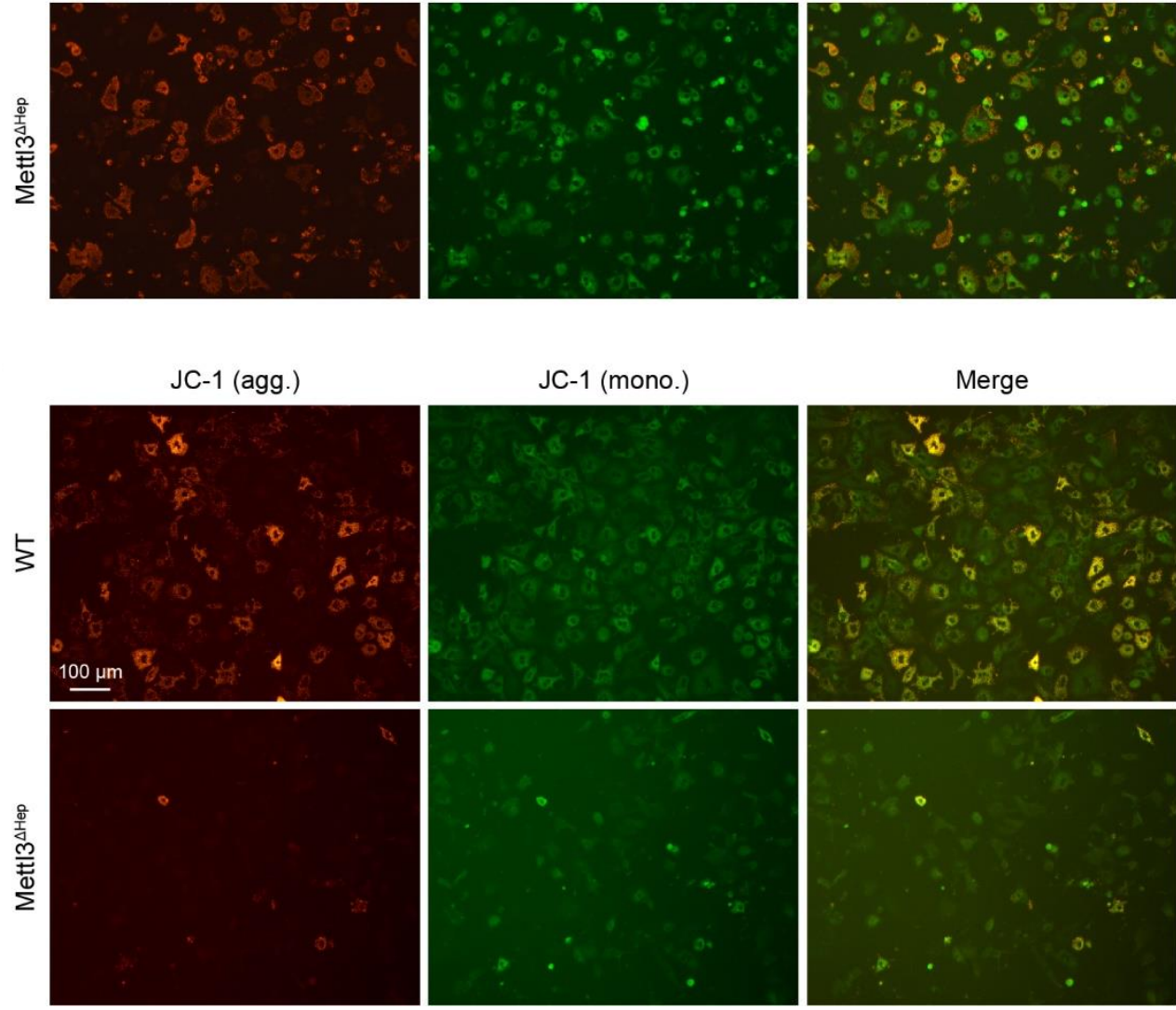

Extended Data Fig. 7: Mett|3 deficiency in hepatocytes causes mitochondrial damage and ER stress

a, Electron microscopy of WT and Mettl $3^{\triangle \text { hep }}$ livers. Arrowheads indicate the perinuclear 
978 space. Scale bar, $5 \mu \mathrm{m}$. b, Mitochondrial membrane potential assessment of primiary 979 hepatocytes from WT and Mettl $3^{\Delta \text { hep }}$ mice 24 hours after isolation with the mitochondria980 specific probe JC-1. Red and green fluorescence indicate J-aggregates and JC-1 981 monomers, respectively. Scale bar, $100 \mu \mathrm{m}$. c, Mitochondrial membrane potential 982 assessment of primiary hepatocytes from WT and Mettl $3^{\Delta \text { hep }}$ mice 48 hours after isolation 983 with the mitochondria-specific probe $\mathrm{JC}-1$. Red and green fluorescence indicate $\mathrm{J}$ 984 aggregates and JC-1 monomers, respectively. Scale bar, $100 \mu \mathrm{m}$.

985 
a

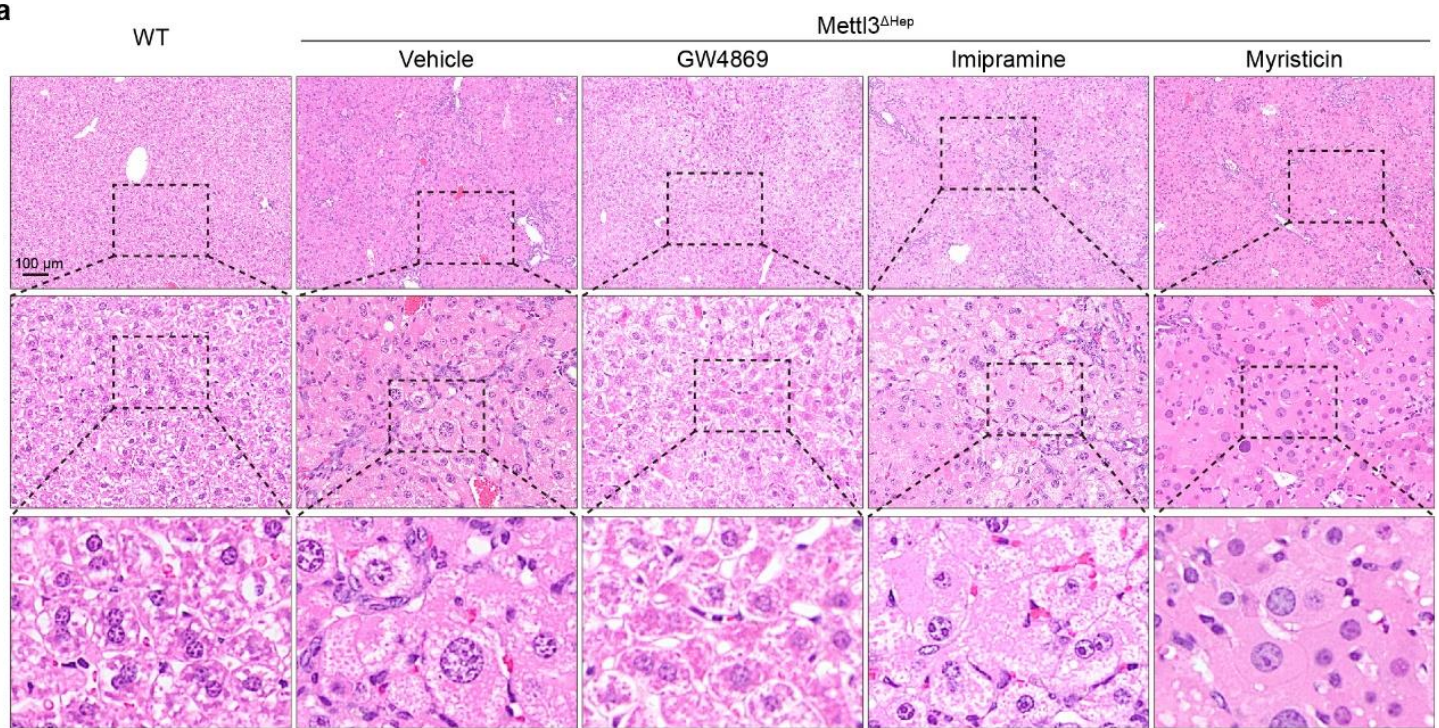

b

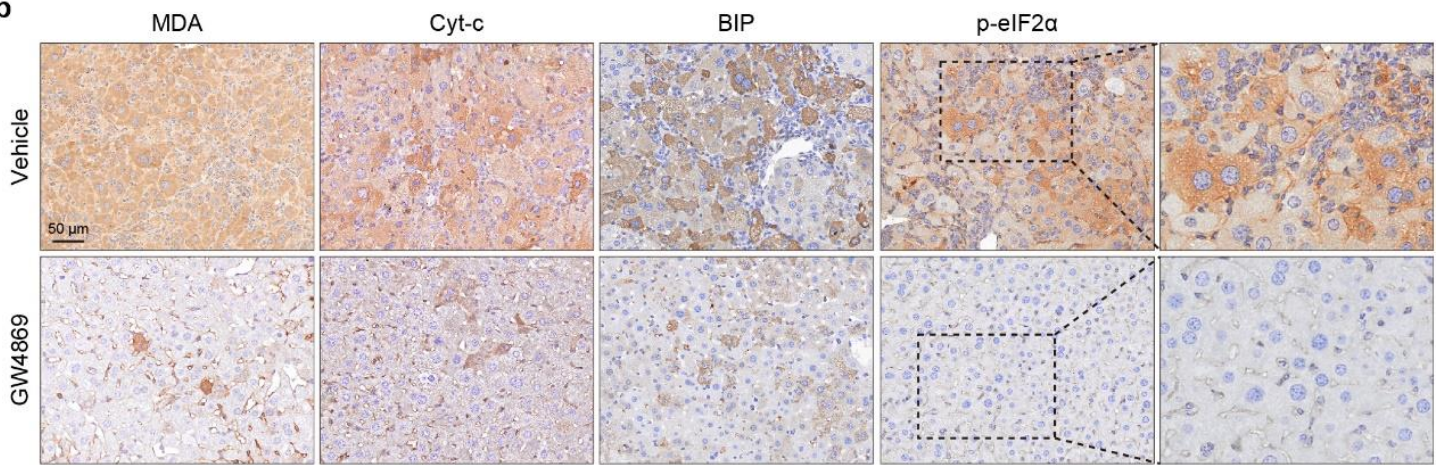

C

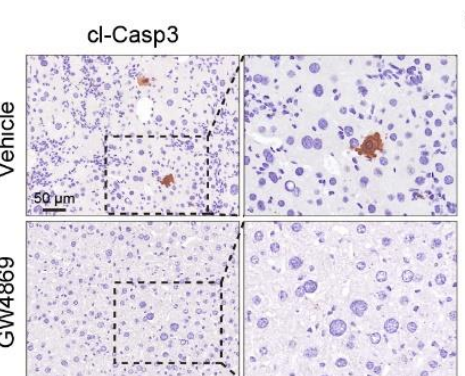

d TUNEL

e $\quad$ CD45

f
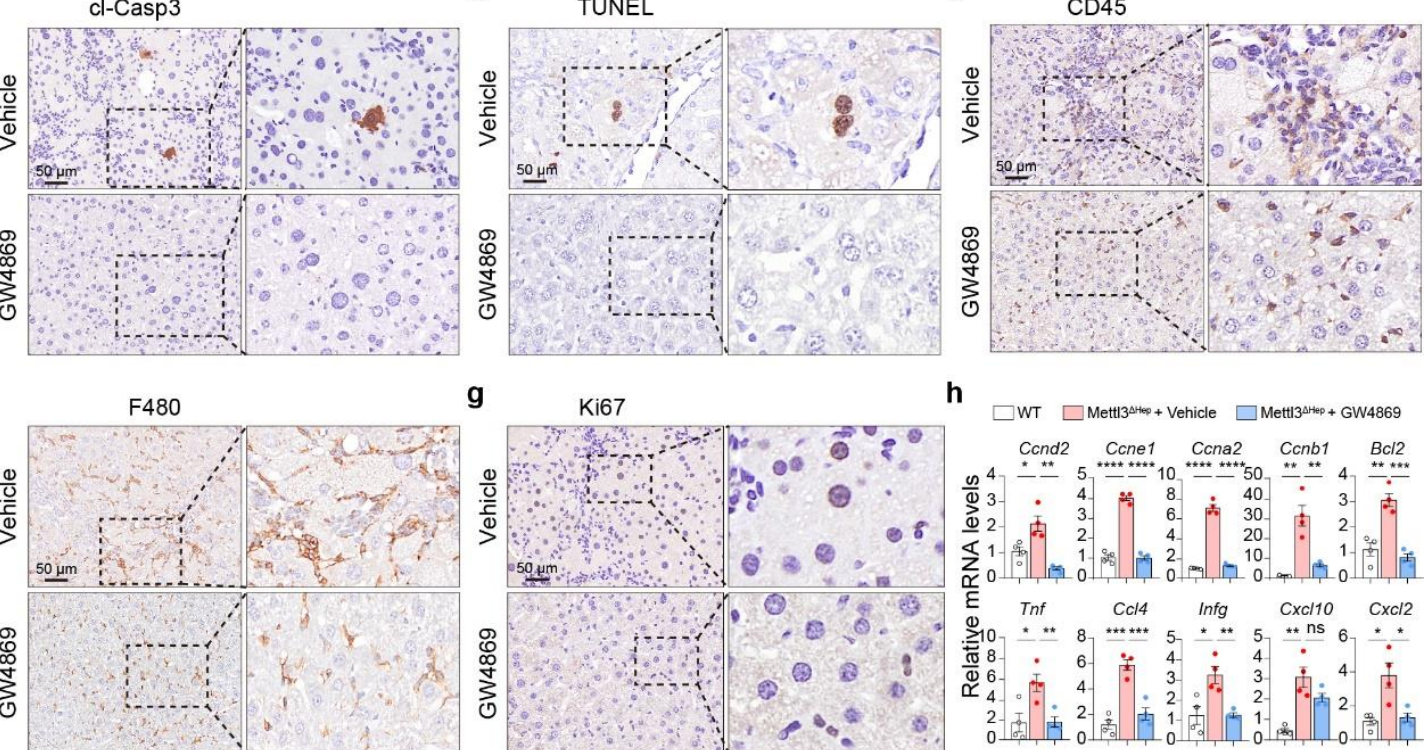

Extended Data Fig. 8: Inhibition of ceramide synthesis by GW4869 ameliorated mitochondrial dysfunction, apoptosis and hepatocyte injury in Mettl $3^{\triangle \text { hep }}$ mice

a, H\&E of WT and Mettl $3^{\text {Ahep }}$ livers treated with vehicle or different inhibitors as indicated. Scale bar, $100 \mu \mathrm{m}$. b, MDA, Cyt-c, BIP and p-elF2 $\alpha$ IHC in Mettl $3^{\triangle \text { hep }}$ livers treated with vehicle versus GW4869. Scale bar, $50 \mu \mathrm{m}$. c-g, IHC of cl-Casp3 (c), TUNEL (d), CD45 (e), F480 (f) and Ki67 (g) in Mettl $3^{\text {Ahep }}$ livers treated with vehicle versus GW4869. Scale bar, 
$99350 \mu \mathrm{m} . \mathbf{h}, \mathrm{qRT}-\mathrm{PCR}$ of Mettl3 ${ }^{\triangle \text { hep }}$ livers treated with vehicle versus GW4869 for indicated

994 genes. Data are shown in mean \pm SEM; ns, not significant, ${ }^{*} p<0.05$, ${ }^{* *} p<0.01$, ${ }^{* * *} p<$ $9950.001,{ }^{* * * *} p<0.0001$ by Student's $t$ test. 
Source Data 1.
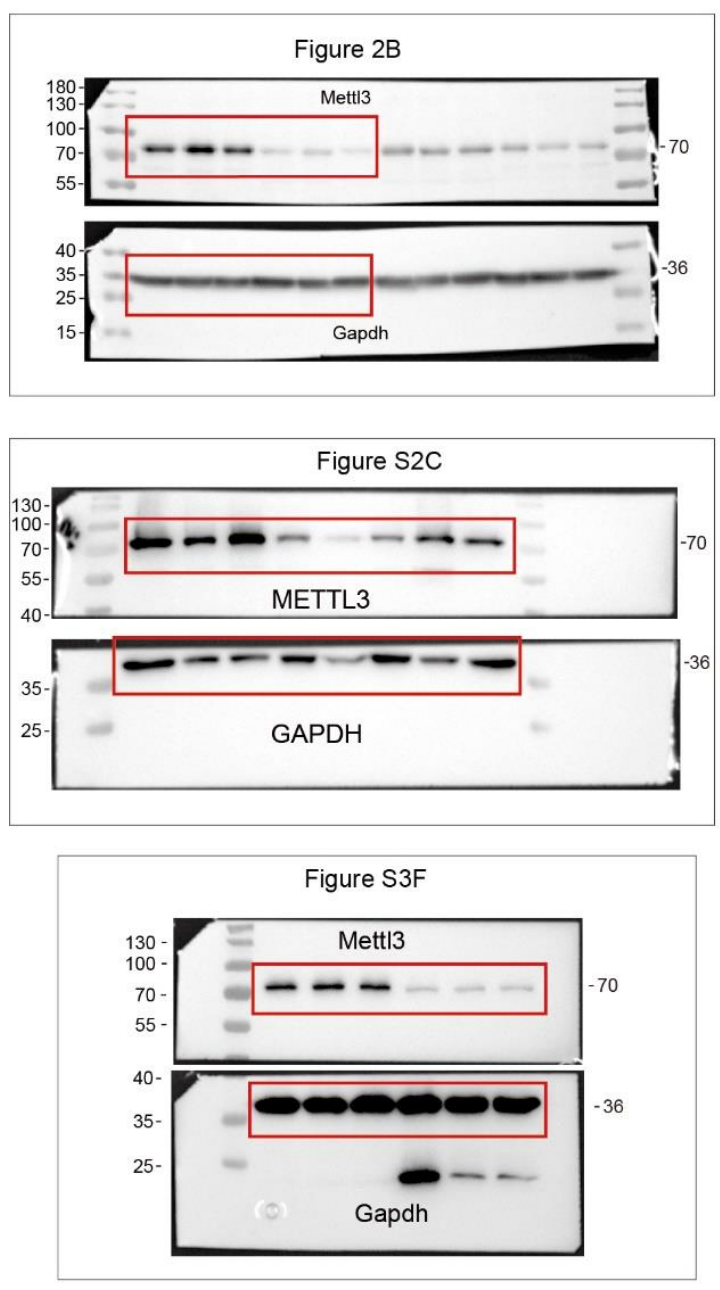

Figure $3 \mathrm{H}$

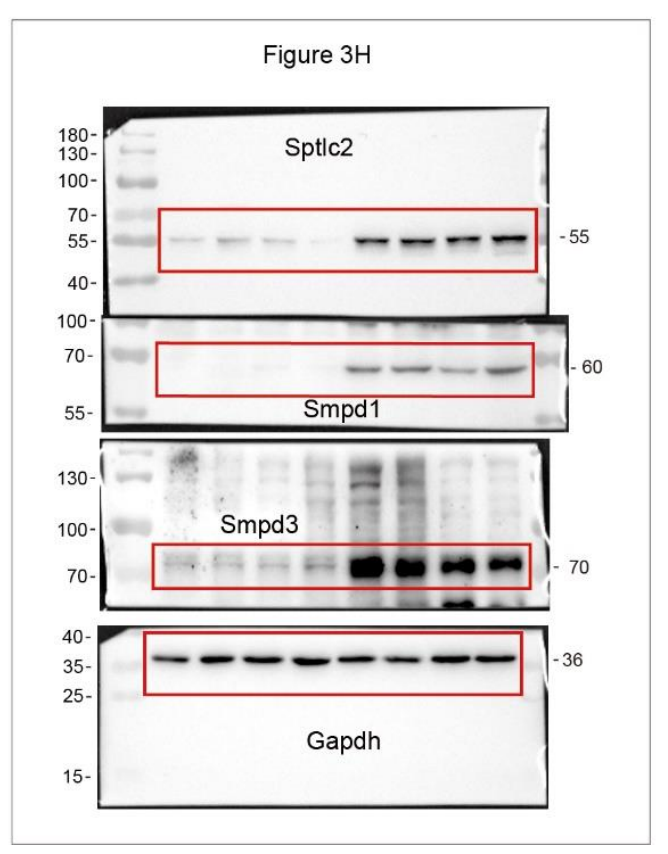

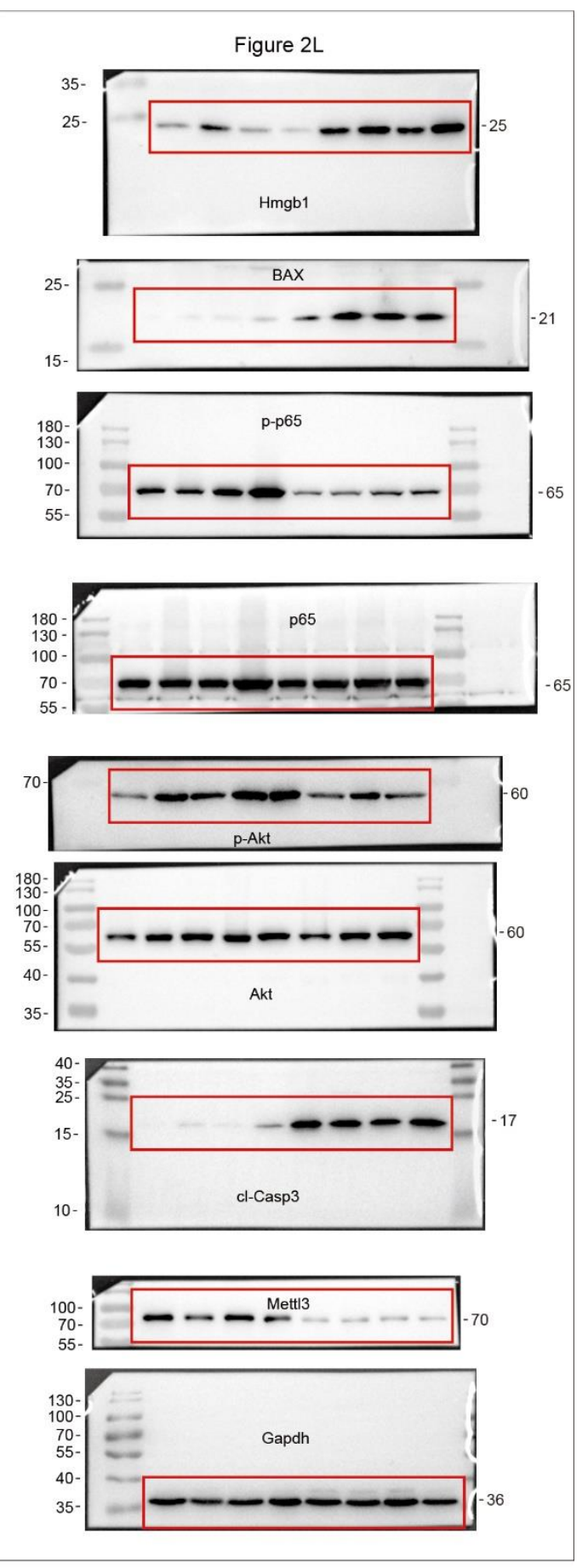


Source Data 2.
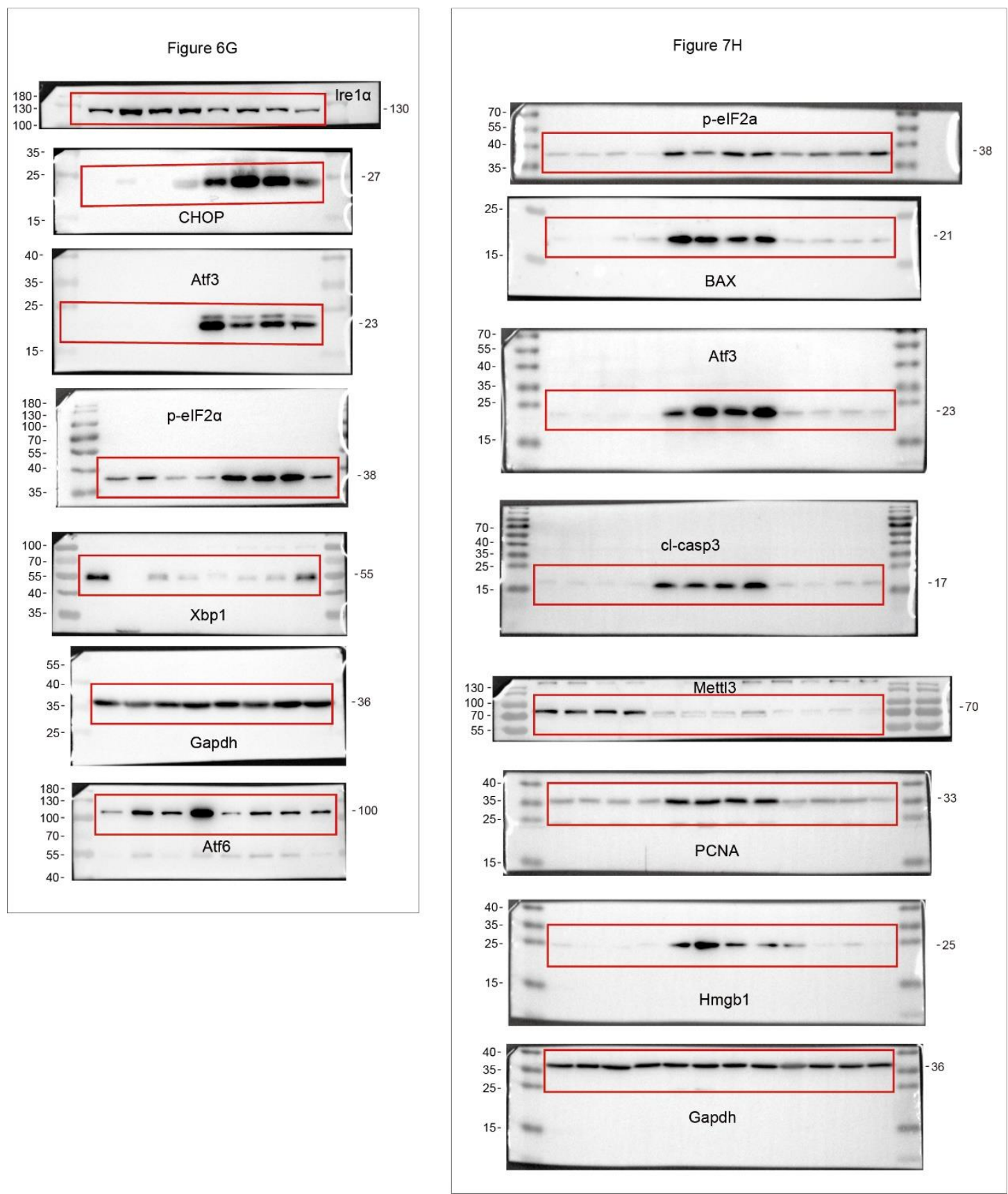


\section{Supplementary Tables}

1002 Supplementary Table 1. Downregulating genes for functional enrichment analysis and network construction

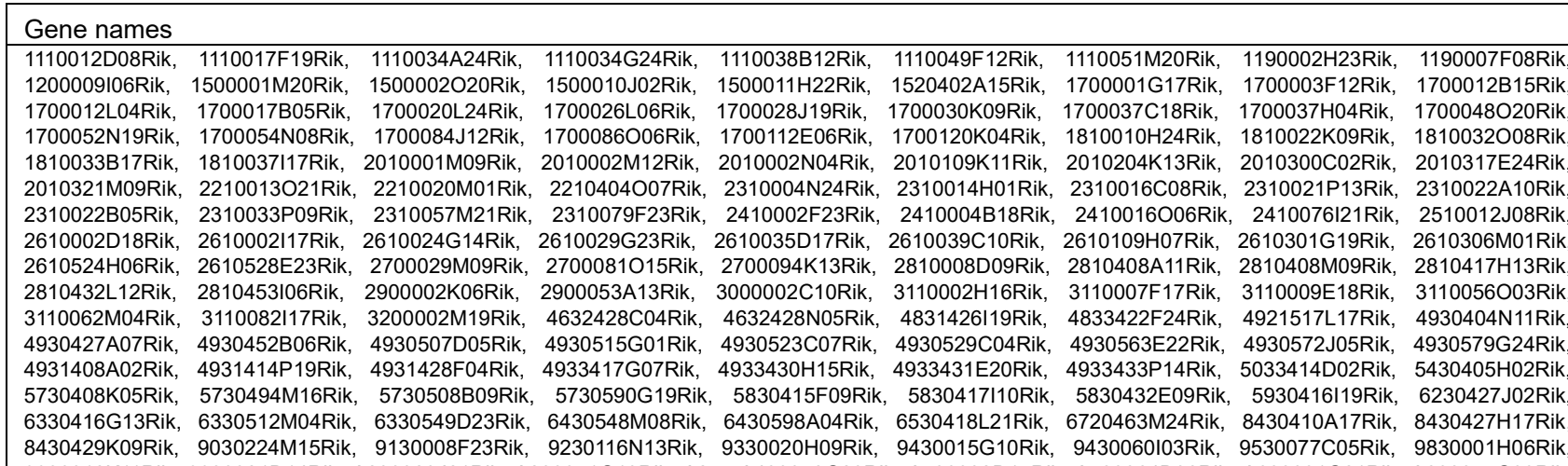
8430429K09Rik, 9030224M15Rik, 9130008F23Rik, 9230116N13Rik, 9330020H09Rik, 9430015G10Rik, 9430060I03Rik, 9530077C05Rik, 9830001H06Rik,
9930012K11Rik, 9930021D14Rik, A230020J21Rik, A230051G13Rik, A2m, A430078G23Rik, A530032D15Rik, A530064D06Rik, A630001G21Rik, A630055G03Rik, A630066F11Rik, A730069N07Rik, A830007P12Rik, AA465934, AA543186, AA986860, Aaas, AB124611, Abca4, Abca7, Abcb10, Abcb9, Abcc1, Abcc5, Abi3, Abl1, Abr, Acap1, Acap3, Ache, Acin1, Acot10, Acot11, Acot9, Acss1, Acta2, Actg2, Actl6a, Acvrl1, Acyp1, Ada, Adam12, Adam15, Adam19, Adam8, Adamts13, Adamts15, Adap1, Adar, Adat1, Adat2, Adat3, Adcy2, Adcy6, Adcy7, Add1, Adora2a, Adora3, Adpgk, Adprh, Adrb1, Adrb2, Adrbk1, Aen, Afap1l1, Afp, Agap2, Agbl5, Agfg1, Agpat1, Agpat4, Agtrap, Ahi1, Al413582, Al427809, Al428936, Al467606, Al662270, Al846148, Aifm3, Ak7, Ak8, Akap8l, Akna, Akr1b10, Akr1b3, Akr1b7, Akr1b8, Alas2, Aldh18a1, Aldh3b1, Aldoart1, Aldoart2, Aldoc, Alkbh1, Alkbh4, Alox12, Alox15, Alox5, Alox5ap, Alpl, Amigo3, Ampd2, Ampd3, Amz1, Anapc1, Anapc11, Ank1, Ankfy1, Ankle1, Ankrd10, Ankrd13a, Ankrd13b, Ankrd13d, Ankrd27, Ankrd35, Ankrd54, Ankrd6, Ankrd9, Anks1, Ano6, Anp32b, Antxr1, Anxa1, Anxa3, Anxa9, Aoah, Aoc2, Ap1g2, Ap2a1, Ap4m1, Apbb1, Apbb3, Apex2, Apitd1, Aplf, Aplp1, Apob48r, Apobec3, Aqp7, Aqp8, Arap3, Arc, Arfgap3, Arfip2, Arhgap1, Arhgap10, Arhgap19, Arhgap22, Arhgap23, Arhgap27, Arhgap33, Arhgap39, Arhgap4, Arhgap9, Arhgdib, Arhgef1, Arhgef17, Arhgef2, Arhgef25, Arhgef4, Arhgef6, Arid3b, Arid5a, Arl10, Arl11, Arl2, Arl2bp, Arl4a, Arl5c, Armc2, Armc6, Armc7, Armc9, Armcx2, Armcx6, Arrb1, Arrb2, Arrdc1, Arrdc2, Art4, Arv1, Asb1, Asb2, Asb3, Asb6, Asf1b, Asns, Asprv1, Atf3, Atg4a, Atg4d, Atp13a2, Atp1a3, Atp1a4, Atp1b2, Atp2a3, Atp2b4, Atp4a, Atp8a2, Atp8b2, Atp8b4, Atpif1, Atxn2l, Atxn7l1, Atxn7l2, Atxn7l3, AU023871, Aurka, Aurkb, AW551984, Axl, Azi1, B230208H17Rik, B230217C12Rik, B230312A22Rik, B3galt4, B3galt6, B3gnt4, B3gnt7, B3gnt8, B430306N03Rik, B4galt2, B4galt3, B9d1, B9d2, Bag2, Bahd1, Baiap3, Bambi-ps1, Bard1, Bat1a, Batf, Bbc3, BC005764, BC017643, BC018242, BC019943, BC021614, BC026590, BC030867, BC037034, BC046331, BC046404, BC048546, BC049762, BC055324, BC057079, BC068157, BC068281, BC088983, Bcas1, Bckdk, Bcl11a, Bcl211, Bcl2111, Bcl2/12, Bcl7c, Bcorl1, Bex1, Bhlha15, Bid, Birc5, Bpgm, Brf1, Brp16, Brpf1, Brpf3, Brsk1, Bsdc1, Bst1, Btbd19, Btf3, Btg2, Btg3, Btk, Btrc, Bub1b, Bud13, BY080835, Bysl, Bzrap1, Bzw2, C130026l21Rik, C130050018Rik, C1qtnf4, C1qtnf6, C230052I12Rik, C2cd3, C330006K01Rik, C330021F23Rik, C5ar1, C630004H02Rik, C85492, C920009B18Rik, Cabin1, Cachd1, Cacna1g, Cacna1h, Cacnb2, Cacnb3, Cad, Camk2a, Camkk1, Camsap1, Camta1, Cand2, Capg, Capn1, Capn12, Capn3, Capn5, Capn6, Car1, Car13, Car2, Car7, Car9, Card14, Card9, Carm1, Casc3, Caskin2, Casp2, Casz1, Cav1, Cblb, Cbx6, Ccdc102a, Ccdc103, Ccdc109b, Ccdc114, Ccdc120, Ccdc123, Ccdc134, Ccdc163, Ccdc23, Ccdc24, Ccdc28b, Ccdc3, Ccdc32, Ccdc40, Ccdc57, Ccdc61, Ccdc69, Ccdc8, Ccdc86, Ccdc88b, Ccdc88c, Ccdc94, Cchcr1, Ccl3, Ccna2, Ccnb1, Ccnb2, Ccnd3, Ccndbp1, Ccne1, Ccnf, Ccnjl, Ccnk, Ccr1, Ccrl2, Ccrn4l, Cd177, Cd226, Cd248, Cd24a, Cd27, Cd2bp2, Cd300a, Cd300lb, Cd300lf, Cd320, Cd33, Cd34, Cd37, Cd3eap, Cd40, Cd47, Cd52, Cd59b, Cd63, Cd68, Cd79a, Cd79b, Cd9, Cd97, Cdan1, Cdc20, Cdc23, Cdc25a, Cdc25b, Cdc42ep2, Cdc42ep3, Cdc42se1, Cdc45, Cdc6, Cdc7, Cdca2, Cdca3, Cdca4, Cdca5, Cdca7, Cdca7l, Cdca8, Cdh24, Cdh26, Cdhr2, Cdk1, Cdk16, Cdk2, Cdk4, Cdk5r1, Cdkn2aipnl, Cdkn2c, Cdkn2d, Cdkn3, Cdr2, Cdt1, Cdyl, Cebpd, Cebpe, Celsr3, Cenpa, Cenph, Cenpi, Cenpl, Cenpm, Cenpn, Cenpo, Cenpp, Cenpt, Cenpw, Cep164, Cep250, Cep72, Cep76, Cercam, Cerk, Chaf1a, Chaf1b, Chchd6, Chd1l, Chd5, Chek2, Cherp, Chi3l1, Chi3l3, Chic2, Chpf, Chrna4, Chst11, Chst12, Chst14, Chtf18, Cic, Cinp, Cirh1a, Cited1, Cited4, Ciz1, Ckap2l, Ckap4, Ckb, Cklf, Ckmt1, Cks1b, Cks2, Clasrp, Cldn15, Cldn23, Clec12a, Clec4a1, Clec4a2, Clec4a3, Clec4d, Clic1, Clip2, Clk2, Clk3, Cln6, Cln8, Clp1, Clspn, Clstn1, Clstn3, Cmas, Cmpk2, Cmtm3, Cmtm7, Cnih2, Cnn2, Cnot3, Cnr2, Cntrob, Col16a1, Col17a1, Col1a1, Col1a2, Col5a1, Col5a2, Col6a1, Col6a2, Col9a3, Comtd1, Cops7b, Coro1a, Coro1c, Coro2b, Coro7, Cotl1, Cox6b2, Cpt1c, Crat, Creb311, Crhr2, Crip1, Crispld2, Crlf3, Crocc, Crtap, Crtc1, Cryba4, Csda, Csdc2, Csf1, Csf2ra, Csf2rb, Csf2rb2, Csf3r, Csnk1e, Csnk2a2, Csrp1, Cst7, Ctbp1, Ctdp1, Ctps, Ctps2, Ctse, Ctsg, Ctsk, Ctu1, Cul7, Cx3cr1, Cxcr2, Cxcr4, Cxcr7, Cxx1c, Cyb561d1, Cyb5r1, Cyb5r4, Cyba, Cybrd1, Cyfip2, Cyp4f16, Cyp4f18, Cyp51, Cyth3, Cyth4, Cytip, D030056L22Rik, D10Wsu102e, D11Wsu47e, D11Wsu99e, D2Ertd750e, D330028D13Rik, D330041H03Rik, D5Ertd605e, D730005E14Rik, D8Ertd82e, D930015E06Rik, Dact3, Dand5, Dapk2, Dapp1, Darc, Dazap1, Dbil5, Dbn1, Dbndd1, Dcaf12, Dcaf15, Dcaf4, Dcbld1, Dchs1, Dck, Dclk2, Dclre1b, Dcp1b, Dctd, Ddah2, Ddb2, Ddit3, Ddit4, Ddx11, Ddx18, Ddx25, Ddx27, Ddx39, Ddx51, Dedd2, Def6, Degs2, Dennd1c, Dennd2c, Dennd4b, Depdc1b, Depdc5, Det1, Dffb, Dfna5, Dgcr14, Dgkd, Dhcr7, Dhodh, Dhrs11, Dhx35, Dhx37, Dhx8, Diras2, Dlg4, Dlg5, Dlgap3, Dlgap5, Dlk1, Dmpk, Dmrt2, Dna2, Dnahc8, Dnaja4, Dnajb1, Dnajc17, Dnajc27, Dnajc8, Dnajc9, Dnase1l1, Dnmt1, Dnmt3b, Dnttip1, Doc2a, Dock2, Dok1, Dok2, Dok3, Dok4, Donson, Dos, Dpf1, Dpf2, Dph2, Dpys12, Dpys15, Dram1, Drosha, Dscc1, Dsn1, Dtwd1, Dtx2, Dtx3, Dusp2, Dusp5, Dusp8, Dusp9, Dut, Dvl2, Dynll1, Dynlt1a, Dynlt1b, Dyrk3, Dysf, E030024N20Rik, E130203B14Rik, E130303B06Rik, E130306D19Rik, E130309D14Rik, E130317F20Rik, E2f1, E2f2, E2f4, E2f8, E330016A19Rik, E4f1, E530011L22Rik, Ear10, Ear12, Ear2, Ebf1, Ebf4, Ebi3, Ecscr, Edc3, Edc4, Eef1e1, Eef2k, Efcab4a, Efemp2, Efhc1, Efna2, Eftud2, Egflam, Ehbp1/1, Ehd2, Eid2, Eif2ak1, Eif2b3, Eif2c2, Elane, Elf4, Elk1, Ell3, Elmo1, Elmo2, Elovl6, Elovl7, Eme1, Emilin1, Emilin2, Emp1, Emp3, Emr1, Endod1, Engase, Entpd1, Entpd7, Ep400, Epb4.1, Epb4.2, Epb4.9, Epdr1, Ephb2, Ephb3, Epor, Eps15l1, Eps813, Ercc2, Ercc4, Ercc8, Espl1, Espn, Esyt1, Evc, Evc2, Evl, Exoc3l, Exog, Exosc1, Exosc3, Exosc6, Exosc8, Eya3, Ezr, F13a1, F2rl1, F2rl2, F2rl3, F630028010Rik, F630043A04Rik, F830116E18Rik, Fads3, Fam100b, Fam101b, Fam105a, Fam109b, Fam110a, Fam117a, Fam118b, Fam125b, Fam129a, Fam129b, Fam131a, Fam132a, Fam132b, Fam160b2, Fam171a2, Fam176b, Fam189b, Fam198b, Fam38a, Fam43a, Fam46c, Fam53b, Fam54a, Fam55b, Fam55c, Fam57a, Fam64a, Fam65a, Fam65b, Fam65c, Fam69b, Fam78a, Fam83d, Fam83g, Fanca, Fancc, Fancd2, Fancg, Fanci, Far2, Fastkd3, Fbf1, Fbl, Fbn1, Fbrs, Fbxl12, Fbxl15, Fbxl19, Fbxl2, Fbxo10, Fbxo34, Fbxo42, Fbxo5, Fbxo7, Fbxw17, Fcer1g, Fcf1, Fcgr1, Fcgr3, Fcgr4, Fcho1, Fcrl1, Fcrls, Fdps, Fdxacb1, Fech, Fen1, Fermt3, Fes, Fev, Fgd2, Fgd3, Fgr, Fhdc1, Fhl1, Fhl3, Fhod1, Fignl1, Fkbp10, Fkbp1b, Flna, Fmnl1, Fmnl3, Fmod, Fn3k, Fn3krp, Fnbp1, Fndc8, Fntb, Fosl2, Foxj1, Foxm1, Foxo3, Foxo4, Foxp4, Foxred2, Fpr1, Fpr2, Frat1, Frat2, Fuca2, Fundc2, Fus, Fut11, Fut7, Fxyd5, Fyn, Fzr1, G3bp1, G6pc3, G6pd2, G6pdx, Gab2, Gabpb1, Gadd45a, Gale, Galnt10, Galnt11, Galnt12, Galnt6, Gapt, Gar1, Garnl3, Gas7, Gas8, Gata1, Gata5, Gatsl3, Gba2, Gcet2, Gcnt1, Gdf10, Gdf3, Gdpd5, Gemin4, Gemin5, Gemin8, Gfap, Gfi1, Gga2, Gga3, Ggt1, Gimap1, Gimap5, Gins1, Gins2, Gins3, Gipc1, Gipc2, Git1, Gja5, Gli1, Gli2, Glipr1, Glipr2, Glt8d2, Gltp, Gltscr1, Gm10052, Gm10069, Gm10136, Gm10345, Gm1060, Gm10653, Gm10857, Gm10941, Gm11517, Gm11545, Gm11968, Gm12034, Gm12657, Gm12839, Gm13139, Gm13298, Gm13315, Gm13476, Gm13498, Gm13889, Gm14005, Gm14085, Gm14501, Gm15455, Gm15645, Gm16381, Gm16515, Gm16516, Gm166, Gm1673, Gm4535, Gm4987, Gm5069, Gm5088, Gm5434, Gm5766, Gm5803, Gm600, Gm6194, Gm6225, Gm6498, Gm6525, Gm6578, Gm6642, Gm6654, Gm6682, Gm6724, Gm6787, Gm6788, Gm71, Gm7104, Gm711, Gm7334, Gm7694, Gm8801, Gm885, Gm8994, Gm9104, Gm962, Gm9790, Gm996, Gmds, Gmeb2, Gmfg, Gmip, Gmnn, Gmpr, Gna15, Gnal, Gnao1, Gnat2, Gnb11, Gnb4, Gng11, Golm1, Gp1ba, Gp1bb, Gp5, Gp9, Gpc1, Gpc3, Gpc6, Gpnmb, Gpr114, Gpr124, Gpr132, Gpr137b, Gpr137b-ps, Gpr153, Gpr176, Gpr183, Gpr35, Gpr44, Gpr56, Gpr84, Gpr97, Gprasp2, Gps2, Gpsm1, Gpsm2, Gpsm3, Gpx3, Gpx7, Gramd1a, Gramd4, Grap2, Grb10, Grhl3, Grik4, Grik5, Grin2c, Grin2d, Gripap1, Grk6, Grwd1, Gse1, Gsg2, Gsn, Gstm5, Gtf2b, Gtf2f1, Gtf2f2, Gtf2ird2, Gtf3c5, Gtpbp1, Gtpbp2, Gtpbp6, Gtse1, Gucy2g, Gyg, Gypa, Gypc, Gys1, H19, H1fx, H2-T24, H2afx, Hace1, Hat1, Haus1, Haus4, Haus5, Haus8, Hba-a2, Hbb-b1, Hbb-b2, Hbq1, Hcfc1, Hck, Hcls1, Hcst, Hdac1, Hdac6, Hdac7, Hdac8, Hdc, Hddc2, Hddc3, Heatr1, Heatr2, Heatr6, Helq, Hemgn, Hexdc, Hexim2, Hey1, Hic1, Hic2, Hif3a, Hinfp, Hip1r, Hipk2, Hira, Hirip3, Hist1h1d, Hist1h1e, Hist1h2ab, Hist1h2ac, Hist1h2ad, Hist1h2ae, Hist1h2af, Hist1h2ag, Hist1h2ah, Hist1h2ai, Hist1h2ak, Hist1h2an, Hist1h2bb, Hist1h2be, Hist1h2bf, Hist1h2bg, Hist1h2bh, Hist1h2bj, Hist1h2bk, Hist1h2bl, Hist1h2bm, Hist1h2bn, Hist1h2bp, Hist1h2br, Hist1h3a, Hist1h3b, Hist1h3c, Hist1h3d, Hist1h3e, Hist1h3f, Hist1h3h, Hist1h3i, Hist1h4a, Hist1h4b, Hist1h4c, Hist1h4d, Hist1h4f, Hist1h4h, Hist1h4i, Hist1h4j, Hist1h4k, Hist1h4m, Hist2h3b, Hist2h3c1, Hist3h2a, Hk1, Hk2, Hk3, Hkdc1, Hlx, Hmbs, Hmga1, Hmgb2, Hmgb3, Hmgcr, Hmgn2, Hmgxb3, Hmha1, Hmox1, Hn1, Homer3, Hoxa1, Hoxa5, Hps1, Hscb, Hsd3b1, Hsh2d, Hspa12b, Hspbap1, Hsph1, Htra2, Htt, Hunk, Hvcn1, Hyal3, I830077J02Rik, Ica1, Icam1, Icam2, Icam4, Idi1, ler3, Ier5, Ier5I, Iffo1, Ifitm6, Ift140, Ift57, Igdcc4, Igf1r, Igf2, Igf2bp2, Ighmbp2, Igsf1, Igsf3, Igsf8, Ihh, Ikbkap, Ikbkg, Ikzf1, II0ra, II10rb, II12rb2, II16, II17d, II17ra, II18rap, II1r2, II1rl2, II1rn, II21r, II28ra, II2rg, II3ra, IIf2, Immp1I, Impa2, Impdh1, Impdh2, Incenp, Ino80e, Inpp5d, Inpp5e, Inpp5j, Inpp5k, Insl3, Ints1, Ints10, Ints5, Ints7, Ints9, Ipo9, Iqcd, Iqce, Iqgap1, Iqgap3, Irak3, Irf8, Isg20, Itga2b, Itga5, Itgam, Itgb2, Itgb3, Itgb7, Itm2a, Itpka, Itpkc, Itpr3, Itprip, Jak3, Jakmip1, Jdp2, Jmjd5, Jmjd7, Josd2, Jrk, Jub, Kank3, Katnb1, Kbtbd12, Kcnab2, Kcng2, Kcnh2, Kcnip3, Kcnj2, Kcnj5, Kcnk13, Kcnmb1, Kcnn4, Kctd10, Kctd11, Kctd13, Kctd14, Kctd20, Kctd5, Kdelc1, Kdelr3, Kdm1a, Kdm2b, Kdm4a, Kdm5c, Kif12, Kif17, Kif18b, Kif20a, Kif21b, Kif22, Kif23, Kif24, Kif2c, Kif3c, Kifc1, Kifc5b, Klc3, Klf1, Klf13, Klf16, Klhl12, Klhl15, Klhl17, Klhl23, Klhl25, Klhl26, Klhl6, Klk8, Klrb1b, Kntc1, Kpna2, Kri1, Krt19, 
Krt80, Krtcap3, Ksr1, Lamb1, Laptm5, Lass5, Lat, Lat2, Lax1, Layn, Lbr, Lca5I, Lck, Lcn2, Lcp2, Ldb1, Ldoc1l, Lefty1, Lenep, Leprel1, Leprel2, Letm2, Lfng, Lgals1, Lgals2, Lgals3, Lgals4, Lgals6, Lgi2, Lhx2, Lig1, Lilrb3, Limd2, Limk1, Limk2, Lin37, Lingo1, Liph, Lipt2, Llgl1, Lmna, Lmnb1, Lmnb2, Lmo2, Loxl2, Loxl3, Lpar2, Lpar5, Lpcat1, Lpcat2, Lpxn, Lrch4, Lrdd, Lrfn4, Lrmp, Lrrc20, Lrrc24, Lrrc25, Lrrc29, Lrrc32, Lrrc33, Lrrc39, Lrrc4, Lrrc47, Lrrc50, Lrrc56, Lrwd1, Lsm3, Lsm7, Lsp1, Lss, Lst1, Ltb4r1, Ltf, Lxn, Ly6c2, Ly6g5b, Lyl1, Lypd1, Lyz1, Lyz2, Lzic, Lzts2, Mad111, Mad2I1, Mad211bp, Madd, Maf1, Maff, Mafk, Mag, Maged2, Mamdc4, Mamstr, Man1c1, Map3k6, Map3k9, Map4k1, Map4k2, Map4k4, Mapk12, Mapk13, Mapk7, Mapkapk3, March2, March3, March8, Marcksl1, Mark2, Mast2, Mast3, Matk, Mau2, Max, Mbd6, Mblac1, Mbnl3, Mboat2, Mbp, Mcam, Mcart1, Mcm10, Mcm2, Mcm3, Mcm3ap, Mcm4, Mcm5, Mcm6, Mcm7, Mcoln2, Mcph1, Mcrs1, Mdfi, Mdga1, Mdm1, Me2, Meaf6, Med12, Med17, Med20, Med22, Med24, Med26, Med30, Med31, Med9, Mef2d, Meg3, Megf6, Megf8, Meis3, Melk, Mesdc1, Mest, Mett111a, Mettl14, Mettl3, Mex3d, Mfap2, Mff, Mfge8, Mfi2, Mfng, Mfsd10, Mfsd2b, Mgat3, Mgl2, Mgst2, Mgst3, Mical1, Micall2, Mid1, Mier2, Miip, Miox, Mir686, Mir715, Mki67, Mkl1, Mknk1, Mkrn1, Mllt1, Mlst8, Mlxip, Mmgt2, Mmp17, Mmp23, Mmp25, Mmp28, Mmp8, Mmp9, Mms22l, Mns1, Mnt, Mobkl2a, Mogat2, Morc2a, Morn4, Mpg, Mphosph6, Mpl, Mpo, Mpp1, Mpp2, Mpzl1, Mrc2, Mrgpre, Mrm1, Mrpl19, Mrps36, Mrto4, Ms4a4c, Ms4a6b, Msh5, Msl3l2, Mt1, Mt2, Mta3, Mtap1s, Mtap6, Mthfd11, Mthfd2, Mtmr11, Mtmr3, Mtr, Mtss1l, Muc4, Mum1, Mutyh, Mvd, Mxd1, Mxd3, Myadm, Mybbp1a, Mybl2, Myc, Myef2, Myh10, Myl6b, Myl7, Myl9, Mylk3, Myo1d, Myo1f, Myo1g, Myo1h, Myo7a, Myo9b, Myof, Mzf1, Naca, Nacad, Nacc1, Naip5, Napsa, Narf, Nat10, Nat14, Nat8l, Nbeal2, Ncapd2, Ncaph, Ncdn, Ncf1, Ncf2, Ncf4, Nckap1l, Nckap5, Nckap5I, Ncoa4, Ncoa5, Ncrna00086, Nde1, Ndn, Ndor1, Ndrg1, Ndrg3, Ndufa4I2, Necab3, Necap2, Nedd9, Neil3, Nek2, Nek3, Nek8, Neu3, Neurl1a, Neurl4, Nfam1, Nfatc1, Nfatc2, Nfatc4, Nfe2, Nfkb2, Nfrkb, Nfya, Nfyc, Ngfrap1, Ngp, Nhej1, Nip7, Nipa1, Nipal2, Nipal3, Nkg7, Nkx2-3, Nle1, Nlgn2, Nlrp1a, Nmb, Nme4, Nmnat3, Nmral1, Nnat, Nob1, Noc4l, Nol9, Nop2, Nop56, Nos3, Notch4, Nova2, Noxa1, Npas1, Npb, Npcd, Npm3, Nprl3, Nrap, Nrarp, Nrf1, Nrgn, Nrip2, Nrm, Nrp, Nsdhl, Nsg1, Nsl1, Nsmce1, Nt5c2, Nt5c3, Nt5c3l, Nt5dc2, Nudt21, Nudt5, Numa1, Numbl, Nup107, Nup133, Nup155, Nup188, Nup205, Nup210, Nup214, Nup37, Nup43, Nup62, Nup85, Nup93, Nupl2, Nusap1, Nutf2, Nxf1, Nxf7, Nxn, Nxt1, Nynrin, Oaz3, Obfc1, Obsl1, Odc1, Odf2, Odz4, Ogfrl1, Oip5, Olfml2b, Omp, Opn3, Orai2, Orc1, Orc5, Orc6, Osbpl5, Osbpl7, Oscp1, Otub2, Otud5, P2rx1, P2ry13, P2ry6, P4ha2, P4htm, Pabpc4, Pacs1, Pacs2, Padi2, Padi4, Pafah1b3, Pak1, Pak4, Pak6, Palm, Pam16, Pan2, Pank4, Panx1, Papln, Paqr6, Parm1, Parp2, Pars2, Parvb, Parvg, Pask, Patz1, Pbk, Pbx2, Pbx4, Pbxip1, Pcbp3, Pcbp4, Pcdha2, Pcdha4, Pcdha9, Pcdhac2, Pcdhga10, Pcdhga12, Pcdhga7, Pcdhgb6, Pcdhgb7, Pcdhgc4, Pcgf1, Pcgf2, Pcid2, Pcif1, Pck2, Pcna, Pcolce2, Pcsk9, Pcyt1b, Pdcd11, Pdcd5, Pdcd7, Pde10a, Pde4a, Pde6d, Pdgfb, Pdk3, Pdlim2, Pdlim4, Pdlim7, Pdrg1, Pdss1, Pdxp, Pdzd4, Pdzk1ip1, Pear1, Peg13, Pelp1, Per1, Pes1, Pf4, Pfkfb3, Pfkfb4, Pfkl, Pfkm, Pfkp, Pgap3, Pglyrp1, Pgs1, Phc1, Phf10, Phf12, Phf19, Phgdh, Phlda2, Phlda3, Phldb1, Phospho1, Phrf1, Phyhip, Pi16, Pias3, Pif1, Piga, Pigf, Pigq, Pih1d2, Pik3cd, Pik3ip1, Pik3r5, Pik3r6, Pilra, Pilrb1, Pim1, Pin1, Pin1-ps1, Pin4, Pip4k2c, Pira4, Pira7, Pitpnm1, Pitrm1, Pkd113, Pkm2, Pkmyt1, Pkn3, Pla2g12a, Pla2g16, Pla2g7, Plac8, Plagl1, Plagl2, Plaur, Plcb2, Plcg2, Pld4, Plek2, Plekha2, Plekhg2, Plekhg5, Plekho1, Plekho2, Plk1, Plp2, Plscr3, Plxdc1, Plxna1, Plxnb3, Plxnd1, Pml, Pmm1, Pmp22, Pms2, Pnck, Pnpla6, Poc1a, Pola2, Pold1, Pold3, Pole, Pole2, Polh, Polm, Polr1a, Polr1b, Polr1e, Polr2a, Polr2c, Polr2d, Polr2h, Polr3d, Polr3gl, Polr3h, Pom121, Pop1, Pop7, Popdc2, Pot1a, Ppan, Ppapdc1b, Ppapdc3, Ppbp, Ppfia4, Ppic, Ppih, Ppil1, Ppil5, Ppip5k1, Ppm1d, Ppm1h, Ppox, Ppp1r10, Ppp1r13l, Ppp1r15a, Ppp1r3d, Ppp1r3f, Ppp1r3g, Ppp1r8, Ppp1r9b, Ppp2r1b, Pprc1, Pqlc3, Praf2, Pram1, Prc1, Prcp, Prdm11, Prdm6, Prdx2, Prelid2, Prex1, Prg2, Prickle3, Prim1, Prim2, Prkar1b, Prkar2b, Prkcd, Prkcdbp, Prkcq, Prkd1, Prkrip1, Prmt1, Prmt2, Prmt3, Prmt5, Prmt6, Procr, Prpf3, Prpf31, Prpf38a, Prr12, Prr15, Prr24, Prr3, Prr5, Prr5l, Prss30, Prss50, Prss8, Prssl1, Prtn3, Prx, Psat1, Psd, Psd4, Psma8, Psmc3ip, Psmd10, Psme2, Psme3, Psph, Psrc1, Pstpip1, Ptcd1, Ptdss2, Ptger1, Ptger3, Ptgr1, Ptgs1, Ptk7, Ptp4a3, Ptpla, Ptpn1, Ptpn18, Ptpn23, Ptpn6, Ptpn7, Ptprcap, Ptprn, Ptprr, Ptpru, Ptrh1, Pvt1, Pwp2, Pycard, Pycr1, Pycr2, Pygo2, Qtrt1, R3hcc1, R74862, Rab11fip5, Rab2b, Rab31, Rab34, Rab35, Rab36, Rab37, Rab38, Rab3d, Rab3gap2, Rab3il1, Rabep2, Rabl2, Rac2, Rac3, Racgap1, Rad1, Rad23a, Rad51, Rad51ap1, Rad5111, Rad52, Rad54l, Rad9, Rae1, Ralgds, Ranbp1, Ranbp10, Rap1gap2, Rapgefl1, Rarg, Rasa3, Rasa4, Rasal3, Rasd1, Rasgrp2, Rasgrp4, Rasip1, Rasl10a, Rassf1, Rassf4, Rassf5, Raver1, Rbbp5, Rbm10, Rbm14, Rbm19, Rbm22, Rbm38, Rbm43, Rbm8a, Rbmxrt, Rbp2, Rcan3, Rcc1, Rcc2, Rcn3, Rcor2, Rcsd1, Rdh11, Rdh12, Rec8, Recql4, Reep1, Reep2, Reep4, Reep5, Relt, Rem2, Renbp, Rere, Retnlg, Rex01, Rfc3, Rfc4, Rfc5, Rftn1, Rfx1, Rfx2, Rg9mtd3, Rgag4, Rgl2, Rgp1, Rgs10, Rgs12, Rgs14, Rgs2, Rhbdf2, Rhd, Rhebl1, Rhoc, Rhof, Rian, Ribc1, Ric8, Rinl, Ripk3, Ripk4, Rltpr, Rmrp, Rn4.5s, Rnaseh2b, Rnf122, Rnf123, Rnf220, Rnf26, Rnf41, Rnps1, Robo3, Rogdi, Rpa1, Rpa2, Rpa3, Rpap1, Rpia, Rpl10, Rpl12, Rpl13, Rpl14, Rpl17, Rpl18a, Rpl21, Rpl2211, Rpl23a, Rpl26, Rpl27a, Rpl3, Rpl30, Rpl31, Rpl34, Rpl35, Rpl36a, Rpl37, Rpl38, Rpl5, Rpl9, Rplp2, Rpp25, Rpph1, Rps15a, Rps16, Rps19-ps3, Rps23, Rps27a, Rps28, Rps29, Rps6ka1, Rps6ka2, Rps6kl1, Rps7, Rps8, Rpusd1, Rpusd2, Rrm1, Rrm2, Rrp12, Rrp1b, Rrp8, Rrp9, Rrs1, Rsad2, Rtcd1, Rtel1, Rtkn, Runx1, Runx2, Runx3, Rusc1, Ruvbl1, Ruvbl2, Rwdd2a, S100a11, S100a6, S100a8, S100a9, S1pr2, S1pr4, Saal1, Sac3d1, Sall2, Samd1, Samd14, Samhd1, Samsn1, Sap25, Sap30, Sarnp, Sart1, Sart3, Sash3, Sbno2, Sc4mol, Scd2, Scmh1, Scml4, Scrib, Sdcbp2, Sec14l1, Sec61a2, Sec61g, Sell, Selm, Selplg, Sema4b, Sema4c, Sema6b, Sema6c, Sema7a, Senp1, Sepn1, Sept1, Sept5, Sept6, Sept8, Serp2, Serpinb1a, Sertad1, Sertad3, Sesn1, Sesn2, Setd1a, Sf3a1, Sf3a2, Sf3a3, Sf3b3, Sf3b4, Sfn, Sfpi1, Sfswap, Sfxn3, Sfxn4, Sgce, Sgol1, Sgsm1, Sh2b2, Sh2b3, Sh2d3c, Sh2d4a, Sh3bgrl3, Sh3bp1, Sh3pxd2b, Sh3tc2, Shank3, Shisa2, Shisa4, Shkbp1, Shq1, Siah1b, Siglec5, Siglece, Sipa1, Sipa112, Sipa1l3, Sirpa, Sirt6, Siva1, Six5, Ska1, Sla, Sla2, Slamf9, Slbp, Slc10a3, Slc11a1, Slc12a9, Slc14a1, Slc15a3, Slc16a10, Slc16a13, Slc16a3, Slc17a9, Slc19a1, Slc1a4, Slc1a5, Slc1a6, Slc20a1, Slc22a4, Slc24a3, Slc25a14, Slc25a37, Slc25a38, Slc25a4, Slc25a43, Slc27a3, Slc28a1, SIc28a2, Slc29a2, Slc2a1, Slc2a3, Slc2a4, Slc2a6, SIc35a4, Slc36a2, Slc37a2, Slc38a1, Slc38a5, Slc38a9, Slc39a5, SIc39a6, SIc41a1, Slc43a1, Slc43a3, Slc44a2, Slc44a3, Slc44a4, Slc4a1, Slc4a5, Slc6a4, Slc6a9, Slc7a1, Slc7a10, Slc7a11, Slc7a5, Slc7a6, Slc7a7, Slc7a8, Slc9a1, Slc9a5, Slco4a1, Slfn1, Slfn14-ps, Slfn2, Slpi, Slx4, Smad3, Smad6, Smarcd1, Smg5, Smn1, Smo, Smox, Smpd4, Smpdl3b, Sms, Smtn, Smyd3, Snapc4, Snca, Sncg, Snhg10, Snip1, Snn, Snrnp25, Snrnp40, Snrpa1, Snrpd1, Snrpe, Snrpf, Snrpg, Snrpn, Snx1, Snx11, Snx12, Snx15, Snx17, Snx20, Snx22, Snx32, Socs1, Solh, Sorcs2, Sorl1, Sox12, Sox13, Sp110, Sp2, Spa17, Spag5, Spata2, Spata24, Spc24, Spc25, Specc1, Spef1, Speg, Sphk1, Spink3, Spire1, Spire2, Spn, Spna1, Spnb1, Spns3, Spon2, Spred3, Sqle, Src, Srcrb4d, Srf, Srgn, Srpk1, Srrt, Srsf3, Srsf7, Ssbp2, Ssbp4, Sstr2, Ssx2ip, St3gal2, St3gal5, St3gal6, St6galnac4, St8sia3, Stag3, Stambpl1, Stat4, Stc2, Steap1, Stfa2l1, Stk10, Stk11ip, Stk19, Stk24, Stk32c, Stmn1, Stom, Strada, Stx11, Stx1a, Stx2, Stxbp1, Sufu, Sugp2, Sult2b1, Supt4h1, Supt7I, Susd1, Suv39h1, Suv420h2, Svep1, Syce2, Syde1, Sykb, Syngr1, Syngr4, Syt5, Szt2, Tab1, Tacc3, Taf1c, Taf2, Taf5, Taf5I, Taf6I, Taf8, Tagap, Tagln, Tagln2, Tal1, Taok2, Tarbp2, Tbc1d1, Tbc1d10b, Tbc1d10c, Tbc1d2, Tbc1d22b, Tbc1d8, Tbce, Tbkbp1, Tbl2, Tbx2, Tbxa2r, Tbxas1, Tcf19, Tcf3, Tcfl1, Tchp, Tcof1, Tcp1112, Tctn3, Tdg, Tdp1, Tead2, Tead4, Telo2, Tes, Tesc, Tfdp1, Tfdp2, Tgfb1, Tgfb1i1, Tgfb3, Tgif2, Tgm4, Thada, Thg1l, Thoc6, Thop1, Ticam2, Tie1, Tifab, Tigd3, Tigd5, Timp1, Tipin, Tjap1, Tk1, Tle6, TIn1, TIr13, Tm4sf5, Tmc4, Tmc6, Tmc8, Tmcc2, Tmco6, Tmem107, Tmem108, Tmem119, Tmem120b, Tmem132a, Tmem138, Tmem149, Tmem150b, Tmem154, Tmem164, Tmem17, Tmem173, Tmem180, Tmem189, Tmem191c, Tmem199, Tmem206, Tmem216, Tmem22, Tmem229b, Tmem231, Tmem26, Tmem39b, Tmem40, Tmem50b, Tmem51, Tmem88, Tmod1, Tmsb10, Tnc, Tnfaip2, Tnfaip8I2, Tnfrsf14, Tnfrsf18, Tnfrsf21, Tnfrsf4, Tnfsf13b, Tnfsf14, Tnfsf9, Tnip2, Tnk1, Tnk2, Tnnc1, Tomt, Tonsl, Top2a, Top3a, Tprn, Tpt1, Tpx2, Traf5, Traf7, Traip, Trem3, Treml1, Treml2, Trim10, Trim27, Trim35, Trim41, Trim45, Trim47, Trim56, Trim59, Trim6, Triobp, Trip10, Trip13, Trmt12, Trmt2a, Trmt61a, Trmt61b, Tro, Troap, Trp53, Trp53bp1, Trp53i13, Trp53rk, Trpm2, Trpm5, Trpv2, Tsc22d4, Tsen15, Tsen54, Tspan11, Tspan13, Tspan15, Tspan32, Tspan33, Tspan5, Tspan6, Tspan8, Tspo2, Tspyl2, Tspyl3, Tssk6, Ttc39a, Ttf2, Tti1, Ttll1, Ttll11, Ttll12, Ttyh3, Tuba8, Tubb1, Tubb5, Tubb6, Tubd1, Tube1, Tubgcp6, Tuft1, Tulp3, Tusc1, Tusc3, Twf2, Twist1, Txlna, Tyms, Tyms-ps, Tyro3, Tyrobp, U2af1, Uap1l1, Uba7, Ubac1, Ubap2, Ubash3a, Ube2c, Ube2e3, Ube2j2, Ube2o, Ube2ql1, Ube2s, Ube2t, Ubiad1, Ubox5, Ubtf, Ubxn11, Uchl1, Uck2, Ucp2, Ufsp1, Uhrf1, Unc119, Unc13a, Unc13d, Unc5a, Unc5cl, Unk, Urb1, Uros, Usmg5, Usp11, Usp20, Usp36, Usp49, Uxt, Vamp5, Vangl1, Vars, Vash1, Vasp, Vav1, Vhl, Vill, Vim, Vopp1, Vpreb1, Vpreb3, Vrk1, Vrk2, Vwce, Vwf, Was, Wbp11, Wbp7, Wdfy4, Wdhd1, Wdr12, Wdr31, Wdr34, Wdr4, Wdr55, Wdr62, Wdr85, Wdr86, Wdr90, Wdyhv1, Wfdc3, Whrn, Whsc2, Wiz, Wnk4, Wnt6, Wrap53, Wtip, Xab2, Xkr5, Xpo7, Xrcc1, Xrcc3, Ydjc, Ypel3, Zbtb3, Zbtb40, Zbtb8b, Zc3h3, Zc3h4, Zc3hc1, Zcchc3, Zcchc5, Zdhhc14, Zdhhc2, Zdhhc24, Zdhhc3, Zdhhc8, Zfat, Zfp13, Zfp142, Zfp212, Zfp213, Zfp276, Zfp282, Zfp296, Zfp335, Zfp341, Zfp346, Zfp362, Zfp382, Zfp384, Zfp408, Zfp414, Zfp446, Zfp498, Zfp511, Zfp521, Zfp526, Zfp553, Zfp57, Zfp593, Zfp628, Zfp647, Zfp651, Zfp653, Zfp668, Zfp687, Zfp688, Zfp689, Zfp7, Zfp703, Zfp740, Zfp775, Zfp777, Zfp783, Zfp82, Zfp821, Zfp827, Zfp865, Zfp94, Zfp956, Zfpm1, Zfyve26, Zfyve27, Zkscan6, Zmynd19, Znhit3, Zpbp2, Zscan2, Zscan22, Zswim1, Zswim3, Zswim4, Zw10, Zyx 


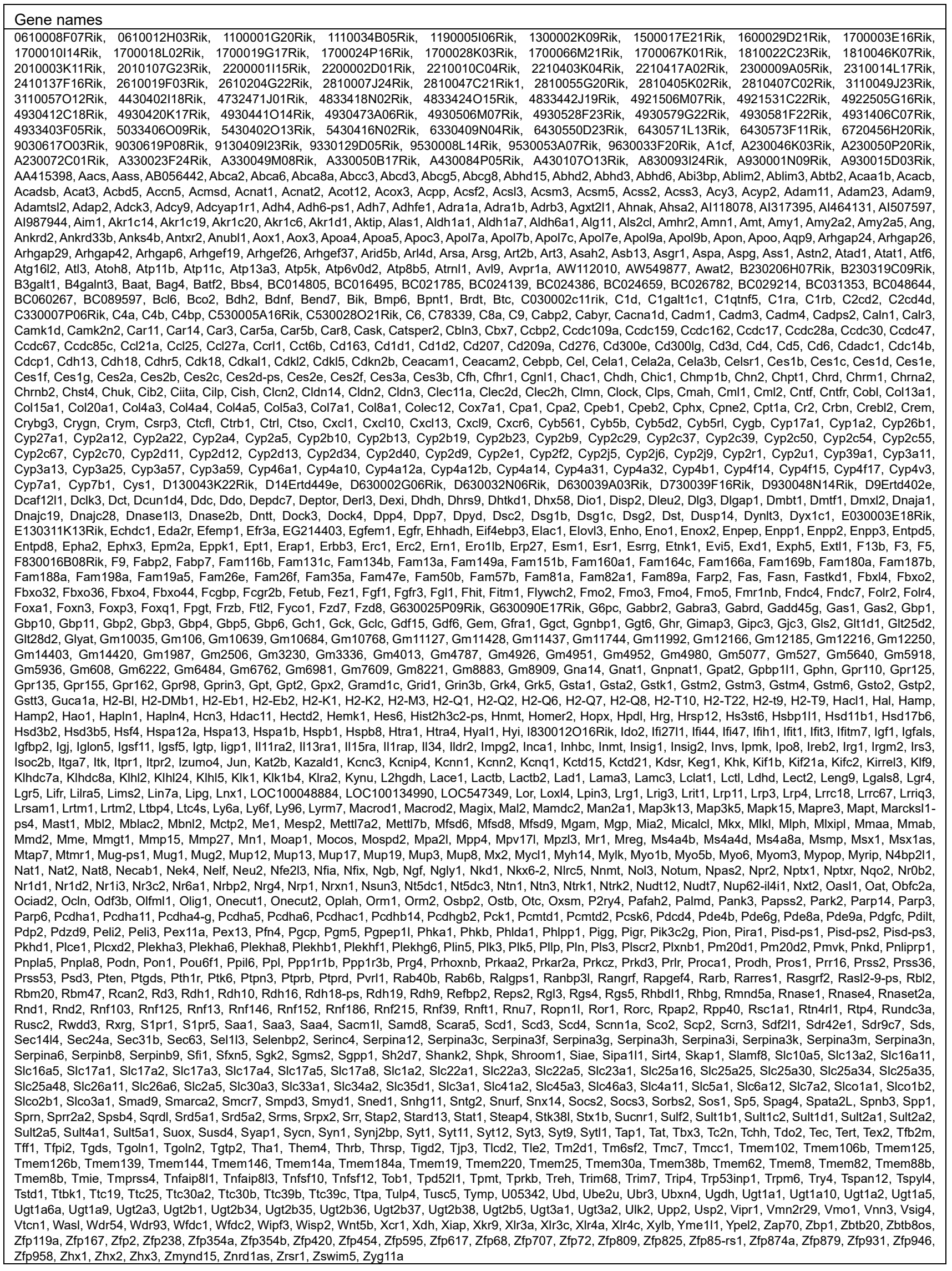



9130206l24Rik, 9230110C19Rik, 9330133014Rik, 9330182L06Rik, 9430020K01Rik, 9430083A17Rik, A330069E16Rik, A430105I19Rik, A430110N23Rik, A530016L24Rik, A530023014Rik, A530088E08Rik, A530099J19Rik, A730011L01Rik, A930018P22Rik, AA474331, Aard, Aatk, AB099516, Abca17, Abca8b, Abca9, Abcc9, Abcd2, Abcg1, Abp1, Accn2, Accs, Ace, Ace2, Acer2, Acot1, Acot2, Acot3, Acot4, Acot5, Acot6, Acsbg1, Actn1, Actn3, Actr3b, Acvr1, Adam22, Adam33, Adamts10, Adamts12, Adamts14, Adamts15, Adamts16, Adamts17, Adamts2, Adamts4, Adamts5, Adamts7, Adamts8, Adamts9, Adamts14, Adarb1, Adc, Adck2, Adcy1, Adcy3, Adcy4, Adcy5, Adm, Adora1, Adrbk2, Afap112, Aff3, Agpat9, Ahdc1, Ahrr, Al429214, Al480653, Al646023, Al661453, Aif1l, Aim1l, Ajap1, Ak1, Ak4, Ak5, Akr1c18, Aldh1a2, Aldh1a3, Aldh112, Aloxe3, Alpk2, Alpk3, Amica1, Amigo2, Amn, Amot11, Anapc4, Angpt17, Ankmy1, Ankrd1, Ankrd23, Ankrd24, Ankrd37, Ankrd39, Ankrd50, Ankrd55, Ankrd56, Anks6, Ano1, Anxa8, Aoc3, Ap1m2, Ap3m2, Apba1, Apc2, Apcdd1, Apcs, Aph1c, Apln, Aplnr, Aptx, Arf2, Arg2, Arhgap28, Arhgap31, Arhgap36, Arhgap44, Arhgap8, Arhgef10, Arhgef15, Arhgef16, Arhgef3, Arl4c, Arrdc4, Arvcf, Asap3, Asb5, Atg9b, At11, Atp11a, Atp13a5, Atp1a2, Atp6v0a4, Atp6v0e2, Atp6v1g2, AU021092, Auts2, AV249152, AW011738, B3gat2, B4galt4, B630019K06Rik, B930041F14Rik, Baalc, Bace1, Bace2, Bambi, BC013712, BC020535, BC022687, BC030307, BC037703, BC057022, BC064078, BC065397, BC090627, BC096441, Bcam, Bcl2a1a, Bcl2a1b, Bcl2a1d, Bcl2114, Bcl6b, Bcmo1, Bcor, Bend5, Bfsp1, Bhlhe22, Blk, Blnk, Bmf, Bmp10, Bmp4, Bmyc, Bnc1, Boc, Bspry, Btbd9, Btg1, C030039L03Rik, C1ql3, C1qtnf2, C1qtnf7, C230081A13Rik, C2cd4c, C3ar1, C630043F03Rik, Cables1, Cacna1a, Cacna1c, Cacna1f, Cacna1s, Cacnb1, Cacng1, Cacng7, Calhm2, Calml4, Camk2b, Cap2, Capsl, Car15, Card10, Card11, Carns1, Casp8, Casq1, Casq2, Cbr2, Cbr3, Cbx2, Cbx4, Ccdc106, Ccdc136, Ccdc160, Ccdc48, Ccdc62, Ccdc64, Ccdc64b, Ccdc78, Ccdc80, Ccdc85b, Ccdc89, Ccdc92, Ccl2, Ccl22, Ccl24, Ccl4, Ccl5, Ccl7, Ccnd1, Ccno, Ccr7, Cd101, Cd14, Cd160, Cd16412, Cd19, Cd2, Cd209f, Cd22, Cd244, Cd28, Cd3g, Cd7, Cd83, Cd8a, Cd96, Cdc42bpg, Cdc42ep5, Cdh11, Cdh3, Cdh4, Cdk20, Cdkn1a, Cdnf, Cdon, Cdr2l, Cds1, Cep68, Chad, Chadl, Chn1, Chrnb1, Chst1, Chst10, Chst15, Chst2, Chst3, Chst7, Chst8, Chsy3, Cidec, Cirbp, Clcf1, Cldn4, Cldn6, Cldn7, Cldn8, Clec10a, Clec14a, Clec3b, Clec9a, Clic3, Clic5, Clic6, Clip3, Clip4, Clstn2, Cma1, Cmklr1, Cnksr1, Cnksr3, Cnn1, Cnnm1, Cnrip1, Cntn2, Col11a2, Col12a1, Col14a1, Col23a1, Col24a1, Col27a1, Col3a1, Col4a1, Col4a6, Col6a6, Col8a2, Corin, Cox4i2, Cox6a2, Cpe, Cpne1, Cpne8, Cpt1b, Cpxm1, Cpxm2, Cpz, Crb2, Creb312, Cry1, Cry2, Cryab, Cspg4, Cspg5, Csrnp1, Csrnp2, Ctbs, Ctf1, Ctgf, Cthrc1, Ctsw, Ctxn1, Cuedc1, Cul9, Cux2, Cx3cl1, Cxcr3, Cxcr5, Cym, Cyp1a1, Cyp2s1, Cyp3a16, Cyp3a41a, Cyp3a44, Cyp4f39, Cysltr2, D030025P21Rik, D0H4S114, D14Ertd668e, D17H6S56E-3, D18Ertd653e, D330050l16Rik, D430019H16Rik, D630003M21Rik, Daam2, Dab1, Dab2, Dab2ip, Dach1, Dact1, Dagla, Dbndd2, Dbp, Dcaf6, Dclk1, Ddc8, Ddit4l, Ddr1, Defb1, Dennd2a, Dennd3, Des, Dgkh, Dhh, Dio3, Dio3os, Disc1, Dkk3, Dlec1, DIl4, Dmkn, Dnahc1, Dnahc17, Dnahc2, Dnaic1, Dnm1, Doc2b, Dpep1, Dpep2, Dpt, Dpy19|3, Dpysl4, Dqx1, Dtna, Dtx1, Dusp1, Dusp18, Dusp4, Dusp6, Dync2li1, Dzip1l, E030010A14Rik, E130012A19Rik, E130309F12Rik, Eaf2, Ecm2, Edn1, Ednra, Efhd1, Efna4, Efna5, Efnb3, Efr3b, Efs, Egfl6, Egfl8, Egln3, Egr1, Egr2, Eid2b, Elf3, Elk3, Eln, Emcn, Emid1, Eml1, Enc1, Endou, Eno2, Eno3, Entpd2, Epb4.112, Epb4.114a, Epcam, Epha1, Epha3, Ephb1, Ephb6, Epn2, Epn3, Eps811, Erbb2, Ereg, Erg, Esrp1, Esrrb, Etv4, Evpl, Exoc6b, Extl3, Eya2, F930015N05Rik, Fabp12, Faim3, Fam101a, Fam102a, Fam107a, Fam110b, Fam110c, Fam113b, Fam115a, Fam115c, Fam123a, Fam129c, Fam13c, Fam151a, Fam161a, Fam164a, Fam167a, Fam171a1, Fam174b, Fam181b, Fam184b, Fam189a1, Fam189a2, Fam46a, Fam46b, Fam71e1, Fam78b, Fam83a, Fam83f, Fam84a, Fam84b, Fam98c, Fblim1, Fbln1, Fbln2, Fbln5, Fbln7, Fbp2, Fbxl16, Fbxl22, Fbxl7, Fbxo17, Fchsd1, Fcrla, Fermt1, Fgd5, Fgf12, Fgf21, Fgf9, Fgfr1, Fhad1, Fhl2, Fhod3, Fibin, Figf, Fjx1, Fkbp5, Flnc, Flt1, Fndc1, Fndc5, Folr1, Fos, Fosb, Fosl1, Foxc1, Foxc2, Foxd2, Foxo6, Foxs1, Fras1, Frem2, Frmd4b, Frmd6, Frs3, Fsd2, Fst, Fst13, Fxyd2, Fxyd3, Fxyd6, Fzd1, Fzd2, Fzd6, Fzd9, G530011006Rik, Gabrp, Gadd45b, Gal3st1, Gal3st2, Galnt11, Galntl4, Gata3, Gatsl2, Gbgt1, Gcc1, Gcom1, Gdf9, Gfpt2, Ggn, Ggt5, Ggt7, Gimap4, Gimap7, Gjb5, Gjc2, Glb11, Glis2, Gm1027, Gm10451, Gm10565, Gm10584, Gm10638, Gm11818, Gm12359, Gm12824, Gm129, Gm13375, Gm13718, Gm14137, Gm14207, Gm14446, Gm14548, Gm3435, Gm347, Gm4902, Gm4925, Gm5113, Gm5523, Gm6644, Gm684, Gm6907, Gm7455, Gm88, Gm9199, Gm9705, Gm9992, Gnb5, Gng7, Gp49a, Gpr120, Gpr133, Gpr157, Gpr18, Gpr30, Gpr39, Gpr4, Gpr55, Gpr68, Gpr81, Gprc5a, Gprc5b, Gpx8, Grap, Grasp, Grhl1, Grhl2, Gria1, Gria3, Grrp1, Gtt2ird1, Gucy2c, Gylt11b, Gzma, Gzmb, Gzmm, H2-DMb2, H2-Oa, H2-Ob, H2afy2, H2afy3, Hand2, Hao2, Hap1, Has3, Hbegf, Hbs1l, Hcn2, Heatr7b1, Hebp2, Heg1, Hes1, Heyl, Hhat, Hist1h2ba, Hoxa2, Hoxa3, Hoxa4, Hoxb2, Hoxb4, Hoxb5, Hoxb7, Hps5, Hpse, Hr, Hrc, Hrct1, Hrh2, Hs2st1, Hs3st1, Hs3st3a1, Hsd11b2, Hsd3b6, Hsf2bp, Hspa1a, Hspb2, Hspb7, Hspg2, Htra3, Id1, Id4, Idua, Iffo2, Ifi27|2a, Ifnar1, Ifngr1, Ift122, Ift172, Igf2bp3, Igfbp1, Igfbp5, Igfbp6, Igsf10, Igsf9, Ikzf3, Ikzf4, II12rb1, II15, II17rb, II17rd, II17re, II18r1, IIa, II1b, II20rb, II22ra1, II27ra, II2rb, II4i1, Ildr1, Inadl, Inhbb, Inhbe, Inpp1, Insl6, Ip6k2, Iqcg, Iqcj-schip1, Iqsec2, Irak1 bp1, Irf4, Irf6, Irgq, Irs2, Isg15, IsIr, Ism1, Ispd, Itga11, Itga9, Itgad, Itgae, Itgal, Itgax, Itgb4, Itpkb, Jag2, Jam2, Jam3, Jph2, Kank4, Kbtbd11, Kbtbd13, Kcna5, Kcna6, Kcnd3, Kcne3, Kcne4, Kcnj10, Kcnj15, Kcnj16, Kcnj8, Kcnk1, Kcnk3, Kcnq4, Kcns3, Kcp, Kctd17, Kif19a, Kif1a, Kif26a, Kif26b, Kin, Kiss1, Kiss1r, Klb, Klf2, Klhl18, Klhl29, KIhl8, Klrb1c KIrd1, Klre1, Klrk1, Krt14, Krt17, Krt222, Krt23, Krt7, L1cam, L3mbtl3, Lag3, Lair1, Lama4, Lama5, Lamb3, Lamc1, Lamc2, Large, Larp6, Lass4, Lass6, Lbh, Lbx2, Ldb2, Ldb3, Ldhb, Lepr, Leprel4, Lgi3, Lgi4, Lhfp, Lhfpl2, Lhx6, Lilra6, Lingo4, Lipo1, Lmcd1, Lmln, Lmod1, Lmtk3, LOC100272221, LOC100499420, LOC100503704, Loxl1, Lpcat4, Lphn1, Lpin1, Lpl, Lrfn1, Lrp12, Lrp2, Lrrc1, Lrrc16a, Lrrc17, Lrrc27, Lrrc46, Lrrc48, Lrrc49, Lrrk1, Lrrn3, Lrrn4, Lrrn4cl, Ltb, Ltb4r2, Ltbp1, Ltbp2, Ltbp3, Ltk, Ly6c1, Ly6d, Ly6g6e, Lynx1, Lysmd2, Mafb, Magee1, Mageh1, Magi2, Mal, Mall, Mamld1, Mansc1, Map3k3, Mapk11, Mapk8ip1, Mapkbp1, March9, Mark1, Marveld2, Marveld3, Mas1, Mcart6, Mdk, Me3, Meis1, Meox1, Mep1b, Metrnl, Mex3a, Mfap4, Mfap5, Mfsd6l, Mfsd7c, Mia1, Micall1, Mill2, Mkrn3, Mks1, Mmp11, Mmp13, Mmp14, Mmp2, Mmp24, Morn1, Mov10, Mpped2, Mpz, Mrgprf, Ms4a7, Msc, Msln Mst1r, Mtl5, Mtus2, Mustn1, Mx1, Mxra8, Mybpc2, Mycbpap, Myct1, Myh11, Myl4, Mylip, Mylpf, Myo18b, Myo7b, Myom1, N4bp3, N6amt1, Naaa, Naif1, Nav1, Nbl1, Ncald, Ncam1, Nccrp1, Nckipsd, Ncr1, Ncrna00085, Ncs1, Ndrg4, Neb, Necab2, Nes, Neurl1b, Neurl2, Neurl3, Nfasc, Nfkb1, Nfkbid, Nfkbie, Ngfr, Nhedc2, Nhlrc1, Niacr1, Nid1, Nid2, Nipal1, Nkain1, Nkain4, Nkapl, Nirc3, Nlrp12, Nirp3, Nme5, Nod2, Nos1ap, Nos2, Notch3, Nov, Noxo1, Npff, Nphp1, Nphp4, Npnt, Nr1h5, Nr4a1, Nradd, Nrp2, Nrtn, Nrxn2, Nsg2, Ntf3, Ntng2, Ntrk3, Nuak1, Nudt11, Nudt17, Numb, Nup62cl, Nupr1, Nxph3, Oas1a, Oas1b, Oas1c, Oas1g, Oas2, Oas3, Oasl2, Obscn, Ogdhl, Olfm1, Olfm2, Olfml3, Ophn1, ORF63, Osbpl10, Osbpl11, Osbpl3, Osbpl6, Ovol1, P2rx2, P2rx3, P2rx6, P2rx7, P2ry14, P2ry2, Pacrg, Pacsin1, Pam, Paqr5, Paqr8, Pard6g, Pax5, Pcdh12, Pcdh18, Pcdha10, Pcdha12, Pcdha3, Pcdha7, Pcdha8, Pcdhb17, Pcdhb19, Pcdhb20, Pcdhb22, Pcdhb4, Pcdhb7, Pcdhga1, Pcdhga3, Pcdhga4, Pcdhga5, Pcdhga6, Pcdhga8, Pcdhga9, Pcdhgb1, Pcdhgb4, Pcdhgb5, Pcdhgc3, Pcdhgc5, Pcnxl2, Pcp4l1, Pcsk4, Pde1a, Pde1b, Pde4c, Pde6c, Pde8b, Pdgfrb, Pdgfrl, Pdk4, Pdlim3, Pdpn, Pdzd7, Pdzrn3, Penk, Per2, Per3, Pfn2, Pgam2, Pgf, Pgm3, Phactr1, Phf11, Phkg1, Pigh, Pigz, Pilrb2, Pim3, Pira11, Pira2, Pira6, Pitpnm2, Piwil2, Pkd1, Pkia, Pknox2, Pkp3, Plat, Plau, Plcd1, Plcd3, Plch1, Plcl1, Plcxd1, Pld1, Pld2, Pld6, Plekha4, Plekhh1, Plekhm2, Plin4, Pls1, Plscr1, Plxdc2, Plxna4, Pmel, Pmepa1, Pnmal2, Pnpla3, Podxl2, Porcn, Pou2af1, Ppargc1b, Ppp1r16b, Ppp1r3c, Pramef8, Prdm16, Prf1, Prickle1, Prickle2, Prkch, Prnd, Prom1, Prox2, Prpf40b, Prph, Prr15l, Prr7, Prrt3, Prrt4, Prss12, Prss35, Ptafr, Ptch2, Ptges, Ptgfr, Ptgfrn, Ptgis, Ptpdc1, Ptpn13, Ptpn14, Ptprm, Ptpro, Pvr, Pvrl4, Pwwp2b, Pxdn, Pygm, Pygo1, Pyroxd2, Qpct, Qrfp, Rab15, Rab19, Rab25, Rab30, Rab3b, Rad9b, Raet1c, Raet1d, Raet1e, Rai14, Ramp1, Ramp3, Rap1gap, Rapgef3, Rapsn, Rasal1, Rasd2, Rasgef1b, Rasgrp1, Rasl10b, Rasl11a, Rasl11b, Rasl12, Rassf10, Rassf9, Rbfox2, Rbfox3, Rcn2, Rdh5, Reck, Relb, Rell2, Reln, Rem1 Rerg, Rftn2, Rgl1, Rgma, Rgnef, Rgs16, Rgs3, Rgs6, Rgs7bp, Rhbdf1, Rhbdl3, Rhoh, Rhoj, Rilpl1, Rims3, Rin1, Ripply3, Rnase10, Rnase6, Rnf157, Rnf165, Rnf208, Rnf222, Rnf43, Rnft2, Rnls, Ror2, Rpgrip1, Rph3al, Rpl34-ps1, Rps27, Rrad, Rsph1, Rsph9, Rspo1, Rtn2, Rtn4rl2, Ryr1, Ryr3, S100a4, S100g, S1pr3, Sall1, Samd10, Samd4, Samd5, Sarm1, Sbk1, Sbsn, Scara3, Scarf2, Sccpdh, Scn1b, Scn3b, Scn4a, Scn4b, Scn8a, Scrn1, Scube3, Scx, Sdk1, Sdk2, Sebox, Sectm1a, Sectm1b, Selp, Sema3b, Sema3f, Sema3g, Sema4f, Sema5b, Sept3, Serac1, Serinc2, Serpina3b, Serpina5, Serpina7, Serpine1, Sertad4, Setd4, Sez6l2, Sfrp1, Sftpd, Sgk1, Sgpp2, Sgsh, Sgsm2, Sh2d2a, Sh3bgr, Sh3bgrl2, Sh3bp4, Sh3d20, Sh3rf2, Sh3tc1, Shc2, Shd, She, Shisa3, Shisa7, Shisa9, Shroom3, Sidt1, Siglec1, Siglecg, Siglech, Sik1, Sike1, Sirpb1b, Ski, Slain1, Slamf6, Slamf7, Slc10a6, Slc12a5, Slc13a3, SIc13a4, SIc15a2, Slc16a4, Slc16a9, SIc1a3, SIc22a17, SIc22a2, Slc23a2, Slc25a29, Slc25a33, Slc26a10, SIc27a1, Slc27a6, Slc29a3, Slc29a4, SIc2a12, SIc31a2, Slc35b3, Slc35e4, Slc35f2, Slc36a4, Slc37a1, Slc43a2, Slc45a2, Slc4a3, Slc4a8, Slc4a9, Slc5a10, Slc5a11, Slc5a2, Slc5a6, Slc6a19, Slc6a8, Slc7a4, Slc8a2, Slc8a3, Slc9a7, Slc9a9, Slit1, Slit3, Smarcd3, Smg6, Smoc2, Smtnl2, Smyd4, Snai1, Snai3, Snhg12, Snhg6, Snord123, Socs5, Sost, Sox17, Sox4, Sox7, Sp100, Sparcl1, Spata6, Spats2l, Spdya, Spib, Spin2, Spint1, Spock2, Spon1, Sprr1a, Spsb1, Sptlc2, Srl, Srpk3, Srpx, Srrm4, St14, St6galnac2, St8sia2, Stab2, Stac2, Stap1, Stard8, Stc1, Stk30, Stk36, Stoml1, Stox1, Sulf1, Susd2, Susd3, Sv2a, Sybu, Syn2, Sync, Synpo, Synpo2, Synpo2l, Sypl2, Syt17, Syt7, Syt12, Sytl3, Sytl4, Tanc1, Taok3, Tbc1d16, Tbc1d19, Tbc1d30, Tbx21, Tcea2, Tceal1, Tceal3, Tcf21, Tcf23, Tcf7, Tcte2, Tctn1, Tctn2, Tecpr1, Tef, Tek, Tep1, Tff3, Tgfbi, Tgm1, Tgtp1, Thbd, Thbs2, Thbs3, Thnsl1, Thsd1, Thy1, Timd4, Timp2, Tln2, TIr1, TIr11, Tlr2, TIr6, TIr9, Tmeff1, Tmem100, Tmem104, Tmem116, Tmem117, Tmem132e, Tmem136, Tmem150c, Tmem151a, Tmem158, Tmem159, Tmem163, Tmem171, Tmem178, Tmem181a, Tmem181b-ps, Tmem181c-ps, Tmem186, Tmem215, Tmem221, Tmem229a, Tmem38a, Tmem44, Tmem45a, Tmem45b, Tmem86a, Tmprss7, Tmprss9, Tmsb15b1, Tmsb15b2, Tmsb15I, Tmtc2, Tnf, Tnfaip6, Tnfrsf10b, Tnfrsf12a, Tnfrsf13c, Tnfrsf19, Tnfrsf22, Tnfrsf23, Tnfsf13, Tnni2, Tnnt1, Tnnt2, Tnrc18, Tns3, Tns4, Tor3a, Tox, Tox2, Tox3, Tpcn2, Tpm2 Tppp3, Tpsb2, Traf1, Treml4, Trib3, Tril, Trim16, Trim46, Trim62, Trnp1, Trp53i11, Trpc3, Trpm4, Trpv4, Tsc22d1, Tsku, Tslp, Tspan18, Tssk1, Ttc12, Ttc17, Ttc26, Ttc28, Ttc30a1, Tt Ttll10, Ttll13, Ttyh1, Tubb4, Tulp2, Twist2, Txk, Txndc16, Tyw3, Ubxn10, Uckl1os, Ugt1a7c, Ulk4, Unc13b, Unc45b, Unc5b, Upk1b, Upk3b, Ush1g, Ushbp1, Usp18, Usp35, Usp43, Vangl2, Vasn, Vav2, Vcan, Vdr, Vegfc, Vil1, Vldlr, Vmn1r90, Vnn1, Vps37d, Vsig10, Vsig2, Vwa1, Wars2, Wbscr17, Wdr19, Wdr41, Wdr72, Wdr82, Wdsub1, Wfdc15b, Wfikkn1, Wif1, Wls, Wnk2, Wnt11, Wnt2, Wnt2b, Wnt4, Wnt5a, Wnt7b, Wnt9b, Wscd1, Wscd2, Wt1, Xaf1, Xcl1, Xirp1, Xkr8, Xkrx, Xlr3b, Xlr4b, Xrra1, Ypel1, Zbed3, Zbtb16, Zbtb32, Zbtb42, Zbtb7c, Zbtb8a, Zcchc18, Zcwpw1, Zdhhc1, Zfand2a, Zfhx2, Zfp128, Zfp14, Zfp184, Zfp202, Zfp27, Zfp275, Zfp286, Zfp3, Zfp30, Zfp316, Zfp319, Zfp334, Zfp385c, Zfp422, Zfp428, Zfp532 Zfp566, Zfp575, Zfp580, Zfp61, Zfp629, Zfp641, Zfp697, Zfp764, Zfp784, Zfp786, Zfp791, Zfp839, Zfp862, Zfpm2, Zfr2, Zkscan4, Zmynd10, Zmynd12 
Supplementary Table 4. Primers for qRT-PCR

\begin{tabular}{|c|c|c|}
\hline Gene & Forward (5' to $\left.3^{\prime}\right)$ & Reverse (5' to $\left.3^{\prime}\right)$ \\
\hline Mett/3 & ATGGGACCAAGGAAGAGTGC & GCCAGGACTCTCAGAATCAACA \\
\hline $1 / 1 a$ & GCACCTTACACCTACCAGAGT & AAACTTCTGCCTGACGAGCTT \\
\hline $1 / 1 b$ & GCAACTGTTCCTGAACTCAACT & ATCTTTTGGGGTCCGTCAACT \\
\hline Tnf & CCCTCACACTCAGATCATCTTCT & GCTACGACGTGGGCTACAG \\
\hline Ifn & ATGAACGCTACACACTGCATC & CCATCCTTTTGCCAGTTCCTC \\
\hline Tgfb1 & CTCCCGTGGCTTCTAGTGC & GCCTTAGTTTGGACAGGATCTG \\
\hline Cxcl2 & CCAACCACCAGGCTACAGG & GCGTCACACTCAAGCTCTG \\
\hline Ccl2 & TTAAAAAACCTGGATCGGAACCAA & GCATTAGCTTCAGATTTACGGGT \\
\hline Cxcl1 & GCCTCTAACCAGTTCCAGCA & AGTGTGGCTATGACTTCGGTTT \\
\hline Cxcl10 & AAGTGCTGCCGTCATTTTCT & CCTATGGCCCTCATTCTCAC \\
\hline Cxcl13 & ATATGTGTGAATCCTCGTGCC A & GGGAGTTGAAGACAGACTTTTGC \\
\hline CCNE1 & GTGGCTCCGACCTTTCAGTC & CACAGTCTTGTCAATCTTGGCA \\
\hline CCNA2 & GCCTTCACCATTCATGTGGAT & TTGCTGCGGGTAAAGAGACAG \\
\hline Col1a1 & ACGCATGAGCCGAAGCTAAC & TTGGGGACCCTTAGGCCATT \\
\hline Col1a2 & GTAACTTCGTGCCTAGCAACA & CCTTTGTCAGAATACTGAGCAGC \\
\hline IL33 & TCCAACTCCAAGATTTCCCCG & CATGCAGTAGACATGGCAGAA \\
\hline Mett/14 & CTGAGAGTGCGGATAGCATTG & GAGCAGATGTATCATAGGAAGCC \\
\hline Wtap & TAGACCCAGCGATCAACTTGT & CCTGTTTGGCTATCAGGCGTA \\
\hline Fto & TTCATGCTGGATGACCTCAATG & GCCAACTGACAGCGTTCTAAG \\
\hline Alkbh5 & CGCGGTCATCAACGACTACC & ATGGGCTTGAACTGGAACTTG \\
\hline Ythdf1 & ACAGTTACCCCTCGATGAGTG & GGTAGTGAGATACGGGATGGGA \\
\hline Ythdf2 & GAGCAGAGACCAAAAGGTCAAG & CTGTGGGCTCAAGTAAGGTTC \\
\hline Ythdf3 & CCTCACCAAGTGCAGTC & GGCACAACACCTAAACCAAA \\
\hline Trp53 & GTCACAGCACATGACGGAGG & TCTTCCAGATGCTCGGGATAC \\
\hline Apaf1 & AGTGGCAAGGACACAGATGG & GGCTTCCGCAGCTAACACA \\
\hline Fas & TATCAAGGAGGCCCATTTTGC & TGTTTCCACTTCTAAACCATGCT \\
\hline Trail & ATGGTGATTTGCATAGTGCTCC & GCAAGCAGGGTCTGTTCAAGA \\
\hline Fasn & GGAGGTGGTGATAGCCGGTAT & TGGGTAATCCATAGAGCCCAG \\
\hline Phyh & CTCGGCCCCAACGATTGTAG & CCCTGGTGGTTTCACCTCC \\
\hline Mgll & CGGACTTCCAAGTTTTTGTCAGA & GCAGCCACTAGGATGGAGATG \\
\hline Fabp1 & ATGAACTTCTCCGGCAAGTACC & CTGACACCCCCTTGATGTCC \\
\hline Pck1 & CTGCATAACGGTCTGGACTTC & CAGCAACTGCCCGTACTCC \\
\hline Ces1d & ATGCGCCTCTACCCTCTGATA & AGCAAATCTCAAGGAGCCAAG \\
\hline Abhd3 & CGTGGGCTTGTCACTGATCTT & AАACТСТССССТССААТСАСТАА \\
\hline Сyp2c44 & GCTGCCCTATACAGATGCCG & GTGACGCTAAGAGTTGCCCA \\
\hline Cyp2e1 & CGTTGCCTTGCTTGTCTGGA & AAGAAAGGAATTGGGAAAGGTCC \\
\hline Acsm5 & CCGATCCCTGAGGTGGTAG & GGTGCCCTGTCTTTTCCAG \\
\hline Smpd1 & TGGGACTCCTTTGGATGGG & CGGCGCTATGGCACTGAAT \\
\hline Smpd2 & TGGGACATCCCCTACCTGAG & TAGGTGAGCGATAGCCTTTGC \\
\hline Smpd3 & TTCTTCGCCAGCCGCTA & CCACCTGCACCTTGAGAAA \\
\hline Sptlc1 & ACGAGGCTCCAGCATACCAT & TCAGAACGCTCCTGCAACTTG \\
\hline Sptlc2 & AACGGGGAAGTGAGGAACG & CAGCATGGGTGTTTCTTCAAAAG \\
\hline Asah1 & CGTGGACAGAAGATTGCAGAA & TGGTGCCTTTTGAGCCAATAAT \\
\hline Cers2 & ATGCTCCAGACCTTGTATGACT & CTGAGGCTTTGGCATAGACAC \\
\hline
\end{tabular}




\begin{tabular}{|l|l|l|}
\hline Cers 4 & GCAGACTCAACGCTGGTTCA & TTGCCTTGACCACAGGAACTG \\
\hline Cers5 & CGGGGAAAGGTGTCTAAGGAT & GTTCATGCAGTTGGCACCATT \\
\hline Degs 1 & GAATGGGTCTACACGGACCAG & CGAGAAGCATCATGGCTACAA \\
\hline Degs 2 & AGCGACTTCGAGTGGGTCTA & TCCCCGTACTAACCAGCAGG \\
\hline Samd8 & GACTCCAACGGCGACTTAGAC & TGCAGAGTTGACTAGGACCTG \\
\hline Sgms1 & GAAGGAAGTGGTTTACTGGTCAC & GACTCGGTACAGTGGGGGT \\
\hline Sgms2 & GAGACAGCAAAACTTGAAGGTCA & CCCGTTGGATAAGGTCTTGGG \\
\hline Atf3 & GAGGATTTTGCTAACCTGACACC & TTGACGGTAACTGACTCCAGC \\
\hline Eif2ak2 & ACGCCAGGTTTAACAGCGAT & TTCTGCCAGCGCTTGTACTT \\
\hline Trib3 & GCAAAGCGGCTGATGTCTG & AGAGTCGTGGAATGGGTATCTG \\
\hline Bcl2 & ATGCCTTTGTGGAACTATATGGC & GGTATGCACCCAGAGTGATGC \\
\hline u-Xbp-1 & GCAGCACTCAGACTATGT & GGTCCAACTTGTCCAGAATGCCC \\
\hline s-Xbp-1 & TGACGAGGTTCCAGAGGTG & TGCACCTGCTGCGGACTCAG \\
\hline Ero1l & GCGTCCAGATTTTCAGCTCT & TCGAAGTGCAAAGGAAATGA \\
\hline Ddit3 & ACCTTCACTACTCTTGACCCTG & GATGTGCGTGTGACCTCTGT \\
\hline Ppp $1 r 15 a-F$ & GAGGGACGCCCACAACTTC & TTACCAGAGACAGGGGTAGGT \\
\hline Cox4i1 & ATTGGCAAGAGAGCCATTTCTAC & CACGCCGATCAGCGTAAGT \\
\hline Gapdh & TGGCCTTCCGTGTTCCTAC & GAGTTGCTGTTGAAGTCGCA \\
\hline Ppargc1a & TATGGAGTGACATAGAGTGTGCT & CCACTTCAATCCACCCAGAAAG \\
\hline
\end{tabular}

